Supplement of Atmos. Chem. Phys., 16, 6931-6947, 2016

http://www.atmos-chem-phys.net/16/6931/2016/

doi:10.5194/acp-16-6931-2016-supplement

(C) Author(s) 2016. CC Attribution 3.0 License.

(c) (i)

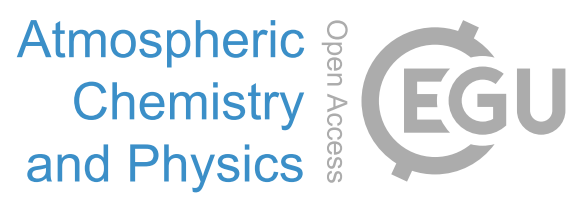

Supplement of

\title{
Global atmospheric budget of simple monocyclic aromatic compounds
}

David Cabrera-Perez et al.

Correspondence to: David Cabrera-Perez (d.cabrera@mpic.de)

The copyright of individual parts of the supplement might differ from the CC-BY 3.0 licence. 
Table 1: Gas phase reactions used in MECCA for the oxidation of aromatic VOC. First column is the enumeration of the equation; the second, labels whether the reactions belong to the troposphere "TR", gas phase "G", contains more than 3 carbon atoms "C"; and nitrogen "N". TEMP correspond to temperature in Kelvin. Detailed description about the labels (and MECCA model) can be found in http://www.rolf-sander.net/messy/mecca/caaba_mecca_manual.pdf. All species and reactions constant are named following MCM nomenclature http://mcm.leeds.ac.uk/MCM/.

\begin{tabular}{|c|c|c|c|c|}
\hline \# & labels & chemical reaction & rate coefficient & reference \\
\hline Aro001 & TrGC & $\mathrm{C} 5 \mathrm{DICARBO} 2+\mathrm{HO}_{2} \rightarrow$ C5DICAROOH & $\begin{array}{l}\text { KR02H02*0.706*(1-rcoch2o2_ } \\
\text { oh-rchohch2o2_oh) }\end{array}$ & $\overline{\text { Rickard and Pascoe }}(\overline{2009})$ \\
\hline Aro002 & $\operatorname{TrGC}$ & $\begin{array}{l}\text { C5DICARBO } 2+\mathrm{HO}_{2} \rightarrow \text { MGLYOX }+ \text { GLYOX }+\mathrm{HO}_{2}+ \\
\text { OH }\end{array}$ & $\begin{array}{l}\text { KRO2H02*0.706*(rcoch2o2_oh-rchohch2o2_ } \\
\text { oh) }\end{array}$ & Rickard and Pascoe $(2009)^{*}$ \\
\hline Aro003 & TrGCN & $\begin{array}{l}\mathrm{C} 5 \mathrm{DICARBO} 2+\mathrm{NO} \rightarrow \mathrm{MGLYOX}+\mathrm{GLYOX}+\mathrm{HO}_{2}+ \\
\mathrm{NO}_{2}\end{array}$ & KRO2NO & Rickard and Pascoe $(2009)^{*}$ \\
\hline Aro004 & TrGCN & $\begin{array}{l}\mathrm{C} 5 \mathrm{DICARBO} 2+\mathrm{NO}_{3} \rightarrow \mathrm{MGLYOX}+\mathrm{GLYOX}+\mathrm{HO}_{2}+ \\
\mathrm{NO}_{2}\end{array}$ & KR02NO3 & Rickard and Pascoe $(2009)^{*}$ \\
\hline Aro005 & $\operatorname{TrGC}$ & $\mathrm{C} 5 \mathrm{DICARBO} 2 \rightarrow \mathrm{MGLYOX}+\mathrm{GLYOX}+\mathrm{HO}_{2}$ & KRO2sHORO2*RO2 & Rickard and Pascoe $(2009)^{*}$ \\
\hline Aro006 & TrGC & $\mathrm{MCOCOMOOOH}+\mathrm{OH} \rightarrow \mathrm{MCOCOMOXO} 2$ & $2.00 \mathrm{E}-11$ & $\overline{\text { Rickard and Pascoe }}(\overline{\overline{2009}})$ \\
\hline Aro007 & TrGC & $\mathrm{C} 33 \mathrm{CO}+\mathrm{OH} \rightarrow \mathrm{CO}+\mathrm{CO}+\mathrm{CO}+\mathrm{HO}_{2}$ & $5.77 \mathrm{E}-11$ & Rickard and Pascoe $(\overline{2009})$ \\
\hline Aro008 & TrGC & $\mathrm{C} 3 \mathrm{DIALOOH}+\mathrm{OH} \rightarrow \mathrm{C} 33 \mathrm{CO}+\mathrm{OH}$ & $1.44 \mathrm{E}-10$ & $\overline{\text { Rickard and Pascoe }}(\overline{\overline{2009}})$ \\
\hline Aro009 & $\operatorname{TrGC}$ & $\mathrm{C} 3 \mathrm{DIALO} 2+\mathrm{HO}_{2} \rightarrow \mathrm{C} 3 \mathrm{DIALOOH}$ & KRO2HO2*0.520*(1-rcoch2o2_oh) & Rickard and Pascoe (2009) \\
\hline Aro010 & $\operatorname{TrGC}$ & $\mathrm{C} 3 \mathrm{DIALO} 2+\mathrm{HO}_{2} \rightarrow \mathrm{GLYOX}+\mathrm{CO}+\mathrm{HO}_{2}+\mathrm{OH}$ & $\mathrm{KRO} 2 \mathrm{HO} 2 * 0.520 * \mathrm{rcoch} 2 \mathrm{o} 2$-oh & $\overline{\text { Rickard and Pascoe }}(\overline{\overline{2009}})^{*}$ \\
\hline Aro011 & TrGCN & $\mathrm{C} 3 \mathrm{DIALO} 2+\mathrm{NO} \rightarrow \mathrm{GLYOX}+\mathrm{CO}+\mathrm{HO}_{2}+\mathrm{NO}_{2}$ & KRO2NO & $\overline{\text { Rickard and Pascoe }}(\overline{2009})^{*}$ \\
\hline Aro012 & TrGCN & $\mathrm{C} 3$ DIALO2 $+\mathrm{NO}_{3} \rightarrow \mathrm{GLYOX}+\mathrm{CO}+\mathrm{HO}_{2}+\mathrm{NO}_{2}$ & KRO2NO3 & $\overline{\text { Rickard and Pascoe }}(\overline{\overline{2009}})^{*}$ \\
\hline Aro013 & TrGC & $\mathrm{C} 3 \mathrm{DIALO} 2 \rightarrow \mathrm{GLYOX}+\mathrm{CO}+\mathrm{HO}_{2}$ & $8.80 \mathrm{E}-13 * \mathrm{R} 02$ & Rickard and Pascoe $(\overline{2009})^{*}$ \\
\hline Aro014 & $\operatorname{TrGC}$ & $\mathrm{HCOCOHCO} 3+\mathrm{HO}_{2} \rightarrow \mathrm{GLYOX}+\mathrm{HO}_{2}+\mathrm{OH}$ & KAPHO $2 *$ rco3_oh & $\overline{\text { Rickard and Pascoe }}(\overline{\overline{2009}})^{*}$ \\
\hline Aro015 & TrGC & $\mathrm{HCOCOHCO} 3+\mathrm{HO}_{2} \rightarrow \mathrm{HCOCOHCO} 3 \mathrm{H}$ & $\mathrm{KAPHO} 2 *\left(\mathrm{rco3} \_\right.$ooh $+\mathrm{rco3} \_$o3 $)$ & Rickard and Pascoe $(\overline{2009})^{*}$ \\
\hline Aro016 & TrGCN & $\mathrm{HCOCOHCO} 3+\mathrm{NO} \rightarrow \mathrm{GLYOX}+\mathrm{HO}_{2}+\mathrm{NO}_{2}$ & KAPNO & Rickard and Pascoe $(\overline{2009})$ \\
\hline Aro017 & TrGCN & $\mathrm{HCOCOHCO} 3+\mathrm{NO}_{2} \rightarrow \mathrm{HCOCOHPAN}$ & k_CH3C03_NO2 & Rickard and Pascoe $(\overline{2009})$ \\
\hline Aro018 & TrGCN & $\mathrm{HCOCOHCO} 3+\mathrm{NO}_{3} \rightarrow \mathrm{GLYOX}+\mathrm{HO}_{2}+\mathrm{NO}_{2}$ & KRO2NO3*1.74 & Rickard and Pascoe $(\overline{2009})$ \\
\hline Aro019 & $\operatorname{TrGC}$ & $\mathrm{HCOCOHCO} 3 \rightarrow \mathrm{GLYOX}+\mathrm{HO}_{2}$ & $\mathrm{KRO} 2 \mathrm{AP} * \mathrm{R} 02$ & Rickard and Pascoe $(\overline{2009})$ \\
\hline Aro020 & $\operatorname{TrGC}$ & $\mathrm{METACETHO}+\mathrm{OH} \rightarrow \mathrm{CH}_{3} \mathrm{C}(\mathrm{O}) \mathrm{OO}$ & $9.82 \mathrm{E}-11$ & Rickard and Pascoe $(\overline{\overline{2009}})$ \\
\hline Aro021 & TrGC & $\mathrm{C} 5$ DIALCO $+\mathrm{OH} \rightarrow$ MALDIALCO $3+\mathrm{CO}$ & $4.90 \mathrm{E}-11$ & Rickard and Pascoe $(\overline{2009})$ \\
\hline Aro022 & TrGCN & $\mathrm{HCOCOHPAN}+\mathrm{OH} \rightarrow \mathrm{GLYOX}+\mathrm{CO}+\mathrm{NO}_{2}$ & $6.97 \mathrm{E}-11$ & Rickard and Pascoe $(\overline{2009})$ \\
\hline Aro023 & TrGCN & $\mathrm{HCOCOHPAN} \rightarrow \mathrm{HCOCOHCO} 3+\mathrm{NO}_{2}$ & k_PAN_M & Rickard and Pascoe $(2009)$ \\
\hline Aro024 & TrGC & $\mathrm{C} 32 \mathrm{OH} 13 \mathrm{CO}+\mathrm{OH} \rightarrow \mathrm{HCOCOHCO} 3$ & $1.36 \mathrm{E}-10$ & $\overline{\text { Rickard and Pascoe }}(\overline{\overline{2009}})$ \\
\hline Aro025 & TrGC & $\mathrm{HCOCOHCO} 3 \mathrm{H}+\mathrm{OH} \rightarrow \mathrm{HCOCOHCO} 3$ & $7.33 \mathrm{E}-11$ & Rickard and Pascoe $(\overline{2009})$ \\
\hline Aro026 & $\operatorname{TrGC}$ & MALANHY + OH $\rightarrow$ MALANHYO2 & $1.4 \mathrm{E}-12$ & Rickard and Pascoe $(\overline{2009})$ \\
\hline Aro027 & $\operatorname{TrGC}$ & $\mathrm{MALDIALOOH}+\mathrm{OH} \rightarrow \mathrm{HOCOC} 4 \mathrm{DIAL}+\mathrm{OH}$ & $1.22 \mathrm{E}-10$ & $\overline{\text { Rickard and Pascoe }}(\overline{\overline{2009}})$ \\
\hline
\end{tabular}


Table 1: Gas phase reactions (... continued)

\begin{tabular}{|c|c|c|c|c|}
\hline \# & labels & chemical reaction & rate coefficient & reference \\
\hline Aro028 & TrGC & MALDIALOOH $+\mathrm{OH} \rightarrow$ MALDIALO2 & $1.90 \mathrm{E}-12 * \operatorname{EXP}(190 / \mathrm{TEMP})$ & Rickard and Pascoe $(2009)$ \\
\hline Aro029 & TrGCN & $\mathrm{NC} 4 \mathrm{DCO} 2 \mathrm{H}+\mathrm{OH} \rightarrow \mathrm{MALANHY}+\mathrm{NO}_{2}$ & $1.90 \mathrm{E}-12 * \operatorname{EXP}(190 / \mathrm{TEMP})$ & $\overline{\text { Rickard and Pascoe }}(\overline{\overline{2009}})^{*}$ \\
\hline Aro030 & TrGC & $\mathrm{CO} 14 \mathrm{O} 3 \mathrm{CO} 2 \mathrm{H}+\mathrm{OH} \rightarrow \mathrm{HCOCH} 2 \mathrm{O} 2$ & $2.19 \mathrm{E}-11$ & Rickard and Pascoe $(\overline{\overline{2009}})$ \\
\hline Aro031 & $\operatorname{TrGC}$ & $\mathrm{BZFUOOH}+\mathrm{OH} \rightarrow \mathrm{BZFUO} 2$ & $3.68 \mathrm{E}-11$ & $\overline{\text { Rickard and Pascoe }}(\overline{\overline{2009}})$ \\
\hline Aro032 & $\operatorname{TrGC}$ & $\mathrm{HOCOC} 4 \mathrm{DIAL}+\mathrm{OH} \rightarrow \mathrm{CO} 2 \mathrm{C} 4 \mathrm{DIAL}+\mathrm{HO}_{2}$ & $3.67 \mathrm{E}-11$ & $\overline{\text { Rickard and Pascoe }}(\overline{\overline{2009}})$ \\
\hline Aro033 & TrGC & MALDIALCO3 $+\mathrm{HO}_{2} \rightarrow$ MALDALCO2H $+\mathrm{O}_{3}$ & KAPHO2*rco3_o3 & Rickard and Pascoe $(\overline{2009})^{*}$ \\
\hline Aro034 & TrGC & MALDIALCO3 $+\mathrm{HO}_{2} \rightarrow$ MALDALCO3H & KAPHO2*rco3_ooh & Rickard and Pascoe $(\overline{2009})^{*}$ \\
\hline Aro035 & TrGC & $\begin{array}{l}\text { MALDIALCO3 }+\mathrm{HO}_{2} \rightarrow .6 \text { MALANHY }+\mathrm{HO}_{2}+.4 \\
\text { GLYOX }+.4 \mathrm{CO}+\mathrm{OH}\end{array}$ & KAPHO2*rco3_oh & $\overline{\bar{R} \text { Rickard and Pascoe }}(\overline{2009})^{*}$ \\
\hline Aro036 & TrGCN & $\begin{array}{l}\text { MALDIALCO3 }+\mathrm{NO} \rightarrow .6 \text { MALANHY }+\mathrm{HO}_{2}+.4 \\
\text { GLYOX }+.4 \mathrm{CO}+\mathrm{NO}_{2}\end{array}$ & KAPNO & Rickard and Pascoe $(2009)^{*}$ \\
\hline Aro037 & $\operatorname{TrGCN}$ & MALDIALCO3 $+\mathrm{NO}_{2} \rightarrow$ MALDIALPAN & k_CH3CO3_NO2 & Rickard and Pascoe (2009) \\
\hline Aro038 & TrGCN & $\begin{array}{l}\text { MALDIALCO3 }+\mathrm{NO}_{3} \rightarrow .6 \text { MALANHY }+\mathrm{HO}_{2}+.4 \\
\text { GLYOX }+.4 \mathrm{CO}+\mathrm{NO}_{2}\end{array}$ & KRO2NO3*1.74 & Rickard and Pascoe $(2009)^{*}$ \\
\hline Aro039 & TrGC & $\begin{array}{l}\text { MALDIALCO } 3 \rightarrow .6 \text { MALANHY }+\mathrm{HO}_{2}+.4 \text { GLYOX }+ \\
.4 \mathrm{CO}\end{array}$ & $\mathrm{KRO2AP} * \mathrm{RO} 2$ & Rickard and Pascoe $(2009)^{*}$ \\
\hline Aro040 & TrGCN & $\mathrm{BZFUONE}+\mathrm{NO}_{3} \rightarrow \mathrm{NBZFUO} 2$ & $3.00 \mathrm{E}-13$ & Rickard and Pascoe (2009) \\
\hline Aro041 & $\operatorname{TrGC}$ & $\begin{array}{l}\text { BZFUONE }+\mathrm{O}_{3} \rightarrow .3125 \mathrm{CO} 14 \mathrm{O} 3 \mathrm{CO} 2 \mathrm{H}+.1875 \\
\mathrm{CO} 14 \mathrm{O} 3 \mathrm{CHO}+.1875 \mathrm{H}_{2} \mathrm{O}_{2}+.5 \mathrm{CO}+.5 \mathrm{HCOCH} 2 \mathrm{O} 2 \\
+.5 \mathrm{OH}\end{array}$ & $2.20 \mathrm{E}-19$ & $\overline{\text { Rickard and Pascoe }}(\overline{2009})^{*}$ \\
\hline Aro042 & TrGC & BZFUONE + OH $\rightarrow$ BZFUO2 & $4.45 E-11$ & Rickard and Pascoe 2009) \\
\hline Aro043 & $\operatorname{TrGCN}$ & $\mathrm{NBZFUOOH}+\mathrm{OH} \rightarrow \mathrm{NBZFUO} 2$ & $6.18 \mathrm{E}-12$ & $\overline{\text { Rickard and Pascoe }}(\overline{\overline{2009}})$ \\
\hline Aro044 & $\operatorname{TrGC}$ & MALDALCO3H $+\mathrm{OH} \rightarrow$ MALDIALCO3 & $4.00 \mathrm{E}-11$ & $\overline{\text { Rickard and Pascoe }}(\overline{\overline{2009}})$ \\
\hline Aro045 & TrGC & $\mathrm{EPXDLCO} 2 \mathrm{H}+\mathrm{OH} \rightarrow \mathrm{C} 3 \mathrm{DIALO} 2$ & $2.31 \mathrm{E}-11$ & Rickard and Pascoe (2009) \\
\hline Aro046 & TrGC & $\mathrm{EPXDLCO} 3+\mathrm{HO}_{2} \rightarrow \mathrm{C} 3 \mathrm{DIALO} 2+\mathrm{OH}$ & KAPHO2*rco3_oh & Rickard and Pascoe $(\overline{2009})^{*}$ \\
\hline Aro047 & TrGC & $\mathrm{EPXDLCO} 3+\mathrm{HO}_{2} \rightarrow$ EPXDLCO2H $+\mathrm{O}_{3}$ & KAPHO2*rco3_o3 & Rickard and Pascoe (2009)* \\
\hline Aro048 & TrGC & $\mathrm{EPXDLCO} 3+\mathrm{HO}_{2} \rightarrow \mathrm{EPXDLCO} 3 \mathrm{H}$ & KAPHO2*rco3_ooh & $\overline{\text { Rickard and Pascoe }}(\overline{\overline{2009}})^{*}$ \\
\hline Aro049 & TrGCN & $\mathrm{EPXDLCO} 3+\mathrm{NO} \rightarrow \mathrm{C} 3 \mathrm{DIALO} 2+\mathrm{NO}_{2}$ & KAPNO & Rickard and Pascoe (2009) \\
\hline Aro050 & TrGCN & $\mathrm{EPXDLCO} 3+\mathrm{NO}_{2} \rightarrow$ EPXDLPAN & k_CH3CO3_NO2 & $\overline{\text { Rickard and Pascoe }}(\overline{\overline{2009}})$ \\
\hline Aro051 & $\operatorname{TrGCN}$ & $\mathrm{EPXDLCO} 3+\mathrm{NO}_{3} \rightarrow \mathrm{C} 3 \mathrm{DIALO} 2+\mathrm{NO}_{2}$ & KR02NO3*1.74 & Rickard and Pascoe $(\overline{2009})$ \\
\hline Aro052 & TrGC & EPXDLCO3 $\rightarrow$ C3DIALO2 & $\mathrm{KRO} 2 \mathrm{AP} * \mathrm{RO} 2$ & Rickard and Pascoe $(\overline{2009})^{*}$ \\
\hline Aro053 & TrGC & $\mathrm{MALNHYOHCO}+\mathrm{OH} \rightarrow \mathrm{CO}+\mathrm{CO}+\mathrm{CO}+\mathrm{HO}_{2}$ & $5.68 \mathrm{E}-12$ & Rickard and Pascoe (2009) \\
\hline Aro054 & TrGCN & MALDIAL $+\mathrm{NO}_{3} \rightarrow$ MALDIALCO3 $+\mathrm{HNO}_{3}$ & $2 * \mathrm{KNO} 03 \mathrm{AL} * 2.0$ & Rickard and Pascoe $(\overline{2009})$ \\
\hline
\end{tabular}


Table 1: Gas phase reactions (... continued)

\begin{tabular}{|c|c|c|c|c|}
\hline$\#$ & labels & chemical reaction & rate coefficient & reference \\
\hline Aro055 & TrGC & $\begin{array}{l}\text { MALDIAL }+\mathrm{O}_{3} \rightarrow 1.0675 \text { GLYOX }+.125 \mathrm{HCHO}+.1125 \\
\mathrm{HCOCO}{ }_{2} \mathrm{H}+.0675 \mathrm{H}_{2} \mathrm{O}_{2}+0.82 \mathrm{HO}_{2}+.57 \mathrm{OH}+1.265 \\
\mathrm{CO}\end{array}$ & $2.00 \mathrm{E}-18$ & Rickard and Pascoe $(\overline{2009})^{*}$ \\
\hline Aro056 & $\operatorname{TrGC}$ & $\begin{array}{l}\text { MALDIAL }+\quad \mathrm{OH} \rightarrow \quad \rightarrow 83 \text { MALDIALCO3 }+.17 \\
\text { MALDIALO2 }\end{array}$ & $5.20 \mathrm{E}-11$ & Rickard and Pascoe (2009)* \\
\hline Aro057 & TrGC & MALANHYOOH $+\mathrm{OH} \rightarrow$ MALNHYOHCO + OH & $4.66 \mathrm{E}-11$ & Rickard and Pascoe (2009) \\
\hline Aro058 & TrGCN & MALDIALPAN $+\mathrm{OH} \rightarrow$ GLYOX $+\mathrm{CO}+\mathrm{CO}+\mathrm{NO}_{2}$ & $3.70 \mathrm{E}-11$ & Rickard and Pascoe (2009) \\
\hline Aro059 & TrGCN & MALDIALPAN $\rightarrow$ MALDIALCO3 $+\mathrm{NO}_{2}$ & k_PAN_M & Rickard and Pascoe (2009) \\
\hline Aro060 & $\operatorname{TrGC}$ & MALANHYO $2+\mathrm{HO}_{2} \rightarrow$ MALANHYOOH & $\begin{array}{l}\text { KRO2HO2*0.625*(1-rcoch2o2 } \\
\text { oh-rchohch2o2_oh) }\end{array}$ & 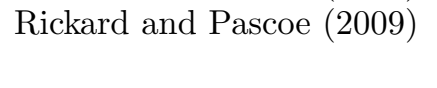 \\
\hline Aro061 & $\operatorname{TrGC}$ & $\mathrm{MALANHYO} 2+\mathrm{HO}_{2} \rightarrow \mathrm{HCOCOHCO} 3+\mathrm{OH}$ & $\begin{array}{l}\mathrm{KRO} 2 \mathrm{HO} 2 * 0.625 *\left(\mathrm{rcoch} 2 \mathrm{o} 2_{-} \mathrm{oh}+\mathrm{rchohch} 2 \mathrm{o} 2_{-}\right. \\
\text {oh) }\end{array}$ & Rickard and Pascoe (2009)* \\
\hline Aro062 & TrGCN & $\mathrm{MALANHYO} 2+\mathrm{NO} \rightarrow \mathrm{HCOCOHCO} 3+\mathrm{NO}_{2}$ & KRO2NO & Rickard and Pascoe (2009)* \\
\hline Aro063 & TrGCN & MALANHYO2 $+\mathrm{NO}_{3} \rightarrow \mathrm{HCOCOHCO} 3+\mathrm{NO}_{2}$ & KRO2NO3 & Rickard and Pascoe (2009)* \\
\hline Aro064 & TrGC & MALANHYO2 $\rightarrow$ HCOCOHCO3 & $8.80 \mathrm{E}-13 * \mathrm{R} 02$ & Rickard and Pascoe $(\overline{2009})^{*}$ \\
\hline Aro065 & TrGC & $\mathrm{EPXDLCO} 3 \mathrm{H}+\mathrm{OH} \rightarrow \mathrm{EPXDLCO} 3$ & $2.62 \mathrm{E}-11$ & Rickard and Pascoe $(\overline{2009})$ \\
\hline Aro066 & TrGC & $\mathrm{CO} 2 \mathrm{C} 4 \mathrm{DIAL}+\mathrm{OH} \rightarrow \mathrm{CO}+\mathrm{CO}+\mathrm{CO}+\mathrm{CO}+\mathrm{HO}_{2}$ & $2.45 \mathrm{E}-11$ & Rickard and Pascoe $(\overline{2009})$ \\
\hline Aro067 & TrGC & $\mathrm{NBZFUO} 2+\mathrm{HO}_{2} \rightarrow \mathrm{NBZFUOOH}$ & KRO2HO2*0.625*(1-rcoch2o2_oh) & Rickard and Pascoe (2009) \\
\hline Aro068 & $\operatorname{TrGC}$ & $\begin{array}{l}\text { NBZFUO2 }+\mathrm{HO}_{2} \rightarrow .5 \mathrm{CO} 14 \mathrm{O} 3 \mathrm{CHO}+.5 \mathrm{NO}_{2}+.5 \\
\text { NBZFUONE }+.5 \mathrm{HO}_{2}+\mathrm{OH}\end{array}$ & KRO2HO2*0.625*rcoch 202 _oh & Rickard and Pascoe $(\overline{2009})^{*}$ \\
\hline Aro069 & TrGCN & $\begin{array}{l}\text { NBZFUO2 }+\mathrm{NO} \rightarrow .5 \mathrm{CO} 14 \mathrm{O} 3 \mathrm{CHO}+.5 \mathrm{NO}_{2}+.5 \\
\text { NBZFUONE }+.5 \mathrm{HO}_{2}+\mathrm{NO}_{2}\end{array}$ & KRO2NO & Rickard and Pascoe $(2009)^{*}$ \\
\hline Aro070 & TrGCN & $\begin{array}{l}\text { NBZFUO2 }+\mathrm{NO}_{3} \rightarrow .5 \mathrm{CO} 14 \mathrm{O} 3 \mathrm{CHO}+.5 \mathrm{NO}_{2}+.5 \\
\text { NBZFUONE }+.5 \mathrm{HO}_{2}+\mathrm{NO}_{2}\end{array}$ & KR02NO3 & Rickard and Pascoe $(2009)^{*}$ \\
\hline Aro071 & TrGCN & $\begin{array}{l}\mathrm{NBZFUO} 2 \rightarrow .5 \mathrm{CO} 14 \mathrm{O} 3 \mathrm{CHO}+.5 \mathrm{NO}_{2}+.5 \mathrm{NBZFUONE} \\
+.5 \mathrm{HO}_{2}\end{array}$ & $8.80 \mathrm{E}-13 * \mathrm{R} 02$ & Rickard and Pascoe $(2009)^{*}$ \\
\hline Aro072 & $\operatorname{TrGC}$ & $\begin{array}{l}\text { MALDALCO2H }+\mathrm{OH} \rightarrow .6 \text { MALANHY }+\mathrm{HO}_{2}+.4 \\
\text { GLYOX }+.4 \mathrm{CO}\end{array}$ & $3.70 \mathrm{E}-11$ & Rickard and Pascoe $(2009)^{*}$ \\
\hline Aro073 & TrGCN & EPXC4DIAL $+\mathrm{NO}_{3} \rightarrow$ EPXDLCO3 $+\mathrm{HNO}_{3}$ & $2 * K N O 3 A L * 4.0$ & Rickard and Pascoe (2009) \\
\hline Aro074 & $\operatorname{TrGC}$ & EPXC4DIAL + OH $\rightarrow$ EPXDLCO3 & $4.32 \mathrm{E}-11$ & Rickard and Pascoe (2009) \\
\hline Aro075 & TrGC & $\mathrm{MECOACETO} 2+\mathrm{HO}_{2} \rightarrow \mathrm{MECOACEOOH}$ & $\mathrm{KRO} 2 \mathrm{HO} 2 * 0.625 *(1-\mathrm{rcoch} 2 \mathrm{o} 2$-oh $)$ & Rickard and Pascoe $(\overline{2009})$ \\
\hline Aro076 & TrGC & $\mathrm{MECOACETO} 2+\mathrm{HO}_{2} \rightarrow \mathrm{CH}_{3} \mathrm{C}(\mathrm{O}) \mathrm{OO}+\mathrm{HCHO}+\mathrm{OH}$ & $\mathrm{KRO} 2 \mathrm{HO} 2 * 0.625 *($ rcoch 202 _oh $)$ & Rickard and Pascoe (2009)* \\
\hline Aro077 & TrGCN & $\mathrm{MECOACETO} 2+\mathrm{NO} \rightarrow \mathrm{CH}_{3} \mathrm{C}(\mathrm{O}) \mathrm{OO}+\mathrm{HCHO}+\mathrm{NO}_{2}$ & KRO2NO & Rickard and Pascoe (2009)* \\
\hline Aro078 & TrGCN & $\mathrm{MECOACETO} 2+\mathrm{NO}_{3} \rightarrow \mathrm{CH}_{3} \mathrm{C}(\mathrm{O}) \mathrm{OO}+\mathrm{HCHO}+\mathrm{NO}_{2}$ & KRO2NO3 & Rickard and Pascoe (2009)* \\
\hline Aro079 & TrGC & $\mathrm{MECOACETO} 2 \rightarrow \mathrm{CH}_{3} \mathrm{C}(\mathrm{O}) \mathrm{OO}+\mathrm{HCHO}$ & KR02pOR02*RO2 & Rickard and Pascoe $(2009)^{*}$ \\
\hline
\end{tabular}


Table 1: Gas phase reactions (... continued)

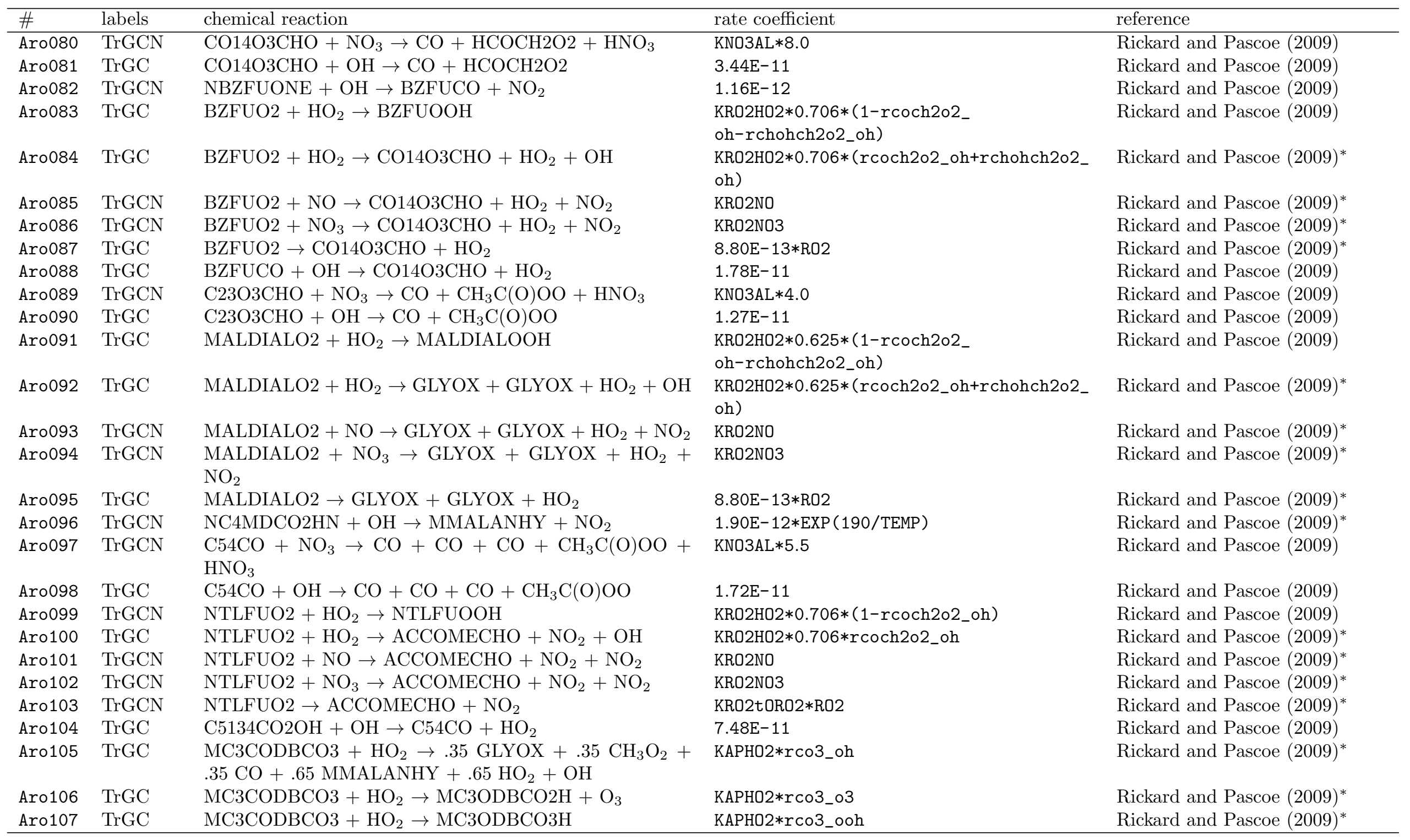


Table 1: Gas phase reactions (... continued)

\begin{tabular}{|c|c|c|c|c|}
\hline \# & labels & chemical reaction & rate coefficient & reference \\
\hline Aro108 & TrGCN & $\begin{array}{l}\mathrm{MC} 3 \mathrm{CODBCO} 3+\mathrm{NO} \rightarrow .35 \mathrm{GLYOX}+.35 \mathrm{CH}_{3} \mathrm{O}_{2}+.35 \\
\mathrm{CO}+.65 \text { MMALANHY }+.65 \mathrm{HO}_{2}+\mathrm{NO}_{2}\end{array}$ & KAPNO & Rickard and Pascoe $(2009)^{*}$ \\
\hline Aro109 & TrGCN & $\mathrm{MC} 3 \mathrm{CODBCO} 3+\mathrm{NO}_{2} \rightarrow \mathrm{MC} 3 \mathrm{CODBPAN}$ & k_CH3CO3_NO2 & Rickard and Pascoe (2009)* \\
\hline Aro110 & TrGCN & $\begin{array}{l}\text { MC3CODBCO3 }+\mathrm{NO}_{3} \rightarrow .35 \mathrm{GLYOX}+.35 \mathrm{CH}_{3} \mathrm{O}_{2}+ \\
.35 \mathrm{CO}+.65 \text { MMALANHY }+.65 \mathrm{HO}_{2}+\mathrm{NO}_{2}\end{array}$ & KRO2NO3*1.74 & Rickard and Pascoe $(\overline{2009})^{*}$ \\
\hline Aro111 & TrGCN & $\begin{array}{l}\text { MC3CODBCO3 } \rightarrow .35 \text { GLYOX }+.35 \mathrm{CH}_{3} \mathrm{O}_{2}+.35 \mathrm{CO}+ \\
.65 \text { MMALANHY }+.65 \mathrm{HO}_{2}\end{array}$ & $1.00 \mathrm{E}-11 * \mathrm{R} 02 * 0.90$ & Rickard and Pascoe $(2009)^{*}$ \\
\hline Aro112 & TrGCN & $\mathrm{MC} 3 \mathrm{CODBCO} 3 \rightarrow \mathrm{MC} 3 \mathrm{ODBCO} 2 \mathrm{H}$ & $1.00 \mathrm{E}-11 * \mathrm{R} 02 * 0.1$ & Rickard and Pascoe $(2009)^{*}$ \\
\hline Aro113 & TrGCN & $\mathrm{C} 5 \mathrm{COO} 2 \mathrm{NO} 2+\mathrm{OH} \rightarrow \mathrm{MGLYOX}+\mathrm{CO}+\mathrm{CO}+\mathrm{NO}_{2}$ & $5.43 E-11$ & Rickard and Pascoe $(\overline{2009})$ \\
\hline Aro114 & TrGCN & $\mathrm{C} 5 \mathrm{COO} 2 \mathrm{NO} 2 \rightarrow \mathrm{C} 5 \mathrm{CO} 14 \mathrm{O} 2+\mathrm{NO}_{2}$ & k_PAN_M & Rickard and Pascoe $(\overline{2009})^{*}$ \\
\hline Aro115 & $\operatorname{TrGC}$ & $\mathrm{C} 5 \mathrm{DIALOOH}+\mathrm{OH} \rightarrow \mathrm{C} 5 \mathrm{DIALCO}+\mathrm{OH}$ & $7.52 \mathrm{E}-11$ & $\overline{\text { Rickard and Pascoe }}(\overline{\overline{2009}})$ \\
\hline Aro116 & $\operatorname{TrGC}$ & $\mathrm{C} 4 \mathrm{CO} 2 \mathrm{DBCO} 3+\mathrm{HO}_{2} \rightarrow \mathrm{C} 4 \mathrm{CO} 2 \mathrm{DCO} 3 \mathrm{H}$ & $\mathrm{KAPHO} 2 *\left(r \mathrm{co} 3 \_\mathrm{ooh}+\mathrm{rco} 3 \_\right.$o3 $)$ & $\overline{\text { Rickard and Pascoe }}(\overline{\overline{2009}})^{*}$ \\
\hline Aro117 & $\operatorname{TrGC}$ & $\mathrm{C} 4 \mathrm{CO} 2 \mathrm{DBCO} 3+\mathrm{HO}_{2} \rightarrow \mathrm{HO}_{2}+\mathrm{CO}+\mathrm{C} 33 \mathrm{CO}+\mathrm{OH}$ & KAPHO2*rco3_oh & Rickard and Pascoe $(\overline{2009})^{*}$ \\
\hline Aro118 & TrGCN & $\mathrm{C} 4 \mathrm{CO} 2 \mathrm{DBCO} 3+\mathrm{NO} \rightarrow \mathrm{HO}_{2}+\mathrm{CO}+\mathrm{C} 33 \mathrm{CO}+\mathrm{NO}_{2}$ & KAPNO & Rickard and Pascoe $(\overline{2009})$ \\
\hline Aro119 & TrGCN & $\mathrm{C} 4 \mathrm{CO} 2 \mathrm{DBCO} 3+\mathrm{NO}_{2} \rightarrow \mathrm{C} 4 \mathrm{CO} 2 \mathrm{DBPAN}$ & k_CH3CO3_NO2 & $\overline{\text { Rickard and Pascoe }}(\overline{\overline{2009}})^{*}$ \\
\hline Aro120 & $\operatorname{TrGCN}$ & $\mathrm{C} 4 \mathrm{CO} 2 \mathrm{DBCO} 3+\mathrm{NO}_{3} \rightarrow \mathrm{HO}_{2}+\mathrm{CO}+\mathrm{C} 33 \mathrm{CO}+\mathrm{NO}_{2}$ & KR02N03*1.74 & Rickard and Pascoe \\
\hline Aro121 & $\operatorname{TrGC}$ & $\mathrm{C} 4 \mathrm{CO} 2 \mathrm{DBCO} 3 \rightarrow \mathrm{HO}_{2}+\mathrm{CO}+\mathrm{C} 33 \mathrm{CO}$ & $\mathrm{KRO} 2 \mathrm{AP} * \mathrm{RO} 2$ & Rickard and Pascoe \\
\hline Aro122 & TrGC & MMALANHY + OH $\rightarrow$ MMALANHYO2 & $1.50 \mathrm{E}-12$ & Rickard and Pascoe $(\overline{\overline{2009}})$ \\
\hline Aro123 & $\operatorname{TrGC}$ & $\mathrm{PXYFUO} 2+\mathrm{HO}_{2} \rightarrow \mathrm{PXYFUOOH}$ & $\begin{array}{l}\text { KRO2HO2*0.706*(1-rcoch } 2 \circ 2_{-} \\
\text {oh-rchohch2o2_oh) }\end{array}$ & $\overline{\text { Rickard and Pascoe }}(\overline{\overline{2009}})$ \\
\hline Aro124 & $\operatorname{TrGC}$ & $\mathrm{PXYFUO} 2+\mathrm{HO}_{2} \rightarrow \mathrm{C} 23 \mathrm{O} 3 \mathrm{CCHO}+\mathrm{HO}_{2}+\mathrm{OH}$ & $\begin{array}{l}\text { KRO2H02*0.706*rcoch2o2_oh }+ \text { rchohch } 202_{-} \\
\text {oh }\end{array}$ & Rickard and Pascoe (2009)* \\
\hline Aro125 & $\operatorname{TrGCN}$ & $\mathrm{PXYFUO} 2+\mathrm{NO} \rightarrow \mathrm{C} 23 \mathrm{O} 3 \mathrm{CCHO}+\mathrm{HO}_{2}+\mathrm{NO}_{2}$ & KRO2NO & Rickard and Pascoe (2009)* \\
\hline Aro126 & TrGCN & $\mathrm{PXYFUO} 2+\mathrm{NO}_{3} \rightarrow \mathrm{C} 23 \mathrm{O} 3 \mathrm{CCHO}+\mathrm{HO}_{2}+\mathrm{NO}_{2}$ & KRO2NO3 & Rickard and Pascoe $(2009)^{*}$ \\
\hline Aro127 & $\operatorname{TrGC}$ & $\mathrm{PXYFUO} 2 \rightarrow \mathrm{C} 23 \mathrm{O} 3 \mathrm{CCHO}+\mathrm{HO}_{2}$ & KRO2tORO2*RO2 & Rickard and Pascoe $(\overline{2009})^{*}$ \\
\hline Aro128 & $\operatorname{TrGC}$ & MMALANHYO $2+\mathrm{HO}_{2} \rightarrow$ MMALNHYOOH & $\begin{array}{l}\text { KRO2HO2*0.706*(1-rcoch } 2 \circ 2_{-} \\
\text {oh-rchohch2o2_oh) }\end{array}$ & $\overline{\text { Rickard and Pascoe }}(\overline{2009})$ \\
\hline Aro129 & $\operatorname{TrGC}$ & MMALANHYO2 $+\mathrm{HO}_{2} \rightarrow \mathrm{CO} 2 \mathrm{H} 3 \mathrm{CO} 3+\mathrm{OH}$ & $\begin{array}{l}\mathrm{KRO} 2 \mathrm{HO} 2 * 0.706 *\left(\mathrm{rcoch} 2 \mathrm{o} 2 \_\mathrm{oh}+\right. \\
\text { rchohch } 2 \mathrm{o} 2 \text { oh })\end{array}$ & Rickard and Pascoe $(2009)^{*}$ \\
\hline Aro130 & $\operatorname{TrGCN}$ & MMALANHYO2 $+\mathrm{NO} \rightarrow \mathrm{CO} 2 \mathrm{H} 3 \mathrm{CO} 3+\mathrm{NO}_{2}$ & KRO2NO & Rickard and Pascoe 2009)* \\
\hline Aro131 & TrGCN & MMALANHYO $2+\mathrm{NO}_{3} \rightarrow \mathrm{CO} 2 \mathrm{H} 3 \mathrm{CO} 3+\mathrm{NO}_{2}$ & KRO2NO3 & Rickard and Pascoe $(\overline{2009})^{*}$ \\
\hline Aro132 & TrGC & MMALANHYO2 $\rightarrow$ CO2H3CO3 & KRO2tORO2*RO2 & Rickard and Pascoe $(\overline{2009})^{*}$ \\
\hline Aro133 & TrGCN & $\mathrm{NPXYFUOOH}+\mathrm{OH} \rightarrow \mathrm{NPXYFUO} 2$ & $5.16 \mathrm{E}-12$ & $\overline{\text { Rickard and Pascoe }}(\overline{\overline{2009}})$ \\
\hline Aro134 & $\operatorname{TrGCN}$ & $\mathrm{C} 23 \mathrm{O} 3 \mathrm{CCHO}+\mathrm{NO}_{3} \rightarrow \mathrm{C} 23 \mathrm{O} 3 \mathrm{CCO} 3+\mathrm{HNO}_{3}$ & KNO3AL $* 5.5$ & Rickard and Pascoe (2009) \\
\hline Aro135 & TrGC & $\mathrm{C} 23 \mathrm{O} 3 \mathrm{CCHO}+\mathrm{OH} \rightarrow \mathrm{C} 23 \mathrm{O} 3 \mathrm{CCO} 3$ & $2.15 \mathrm{E}-11$ & Rickard and Pascoe $(\overline{2009})$ \\
\hline
\end{tabular}


Table 1: Gas phase reactions (... continued)

\begin{tabular}{|c|c|c|c|c|}
\hline \# & labels & chemical reaction & rate coefficient & reference \\
\hline Aro136 & TrGCN & $\mathrm{C} 4 \mathrm{CO} 2 \mathrm{DBPAN}+\mathrm{OH} \rightarrow \mathrm{C} 33 \mathrm{CO}+\mathrm{CO}+\mathrm{NO}_{2}$ & $2.74 \mathrm{E}-11$ & Rickard and Pascoe $(2009)$ \\
\hline Aro137 & TrGCN & $\mathrm{C} 4 \mathrm{CO} 2 \mathrm{DBPAN} \rightarrow \mathrm{C} 4 \mathrm{CO} 2 \mathrm{DBCO} 3+\mathrm{NO}_{2}$ & k_PAN_M & $\overline{\text { Rickard and Pascoe }}(\overline{\overline{2009}})^{*}$ \\
\hline Aro138 & $\operatorname{TrGC}$ & $\begin{array}{l}\mathrm{C} 5 \mathrm{CO} 14 \mathrm{O} 2+\mathrm{HO}_{2} \rightarrow .83 \mathrm{MALANHY}+.83 \mathrm{CH}_{3} \mathrm{O}_{2}+.17 \\
\text { MGLYOX }+.17 \mathrm{HO}_{2}+.17 \mathrm{CO}+\mathrm{OH}\end{array}$ & KAPHO2*rco3_oh & $\overline{\text { Rickard and Pascoe }}(\overline{2009})^{*}$ \\
\hline Aro139 & TrGC & $\mathrm{C} 5 \mathrm{CO} 14 \mathrm{O} 2+\mathrm{HO}_{2} \rightarrow \mathrm{C} 5 \mathrm{CO} 14 \mathrm{OH}+\mathrm{O}_{3}$ & KAPHO2 $*$ rco3_o3 & Rickard and Pascoe $(2009)^{*}$ \\
\hline Aro140 & TrGC & $\mathrm{C} 5 \mathrm{CO} 14 \mathrm{O} 2+\mathrm{HO}_{2} \rightarrow \mathrm{C} 5 \mathrm{CO} 14 \mathrm{OOH}$ & KAPHO2*rco3_ooh & Rickard and Pascoe $(\overline{2009})^{*}$ \\
\hline Aro141 & $\operatorname{TrGCN}$ & $\begin{array}{l}\mathrm{C} 5 \mathrm{CO} 14 \mathrm{O} 2+\mathrm{NO} \rightarrow .83 \mathrm{MALANHY}+.83 \mathrm{CH}_{3} \mathrm{O}_{2}+.17 \\
\text { MGLYOX }+.17 \mathrm{HO}_{2}+.17 \mathrm{CO}+\mathrm{NO}_{2}\end{array}$ & KAPNO & Rickard and Pascoe $(2009)^{*}$ \\
\hline Aro142 & TrGCN & $\mathrm{C} 5 \mathrm{CO} 14 \mathrm{O} 2+\mathrm{NO}_{2} \rightarrow \mathrm{C} 5 \mathrm{COO} 2 \mathrm{NO} 2$ & k_CH3CO3_NO2 & Rickard and Pascoe $(2009)^{*}$ \\
\hline Aro143 & TrGCN & $\begin{array}{l}\mathrm{C} 5 \mathrm{CO} 14 \mathrm{O} 2+\mathrm{NO}_{3} \rightarrow .83 \mathrm{MALANHY}+.83 \mathrm{CH}_{3} \mathrm{O}_{2}+.17 \\
\text { MGLYOX }+.17 \mathrm{HO}_{2}+.17 \mathrm{CO}+\mathrm{NO}_{2}\end{array}$ & KRO2NO3*1.74 & $\overline{\text { Rickard and Pascoe }}(\overline{\overline{2009}})^{*}$ \\
\hline Aro144 & $\operatorname{TrGC}$ & $\begin{array}{l}\mathrm{C} 5 \mathrm{CO} 14 \mathrm{O} 2 \rightarrow .83 \text { MALANHY }+.83 \mathrm{CH}_{3} \mathrm{O}_{2}+.17 \\
\text { MGLYOX }+.17 \mathrm{HO}_{2}+.17 \mathrm{CO}\end{array}$ & $\mathrm{KRO} 2 \mathrm{AP} * \mathrm{RO} 2$ & Rickard and Pascoe $(2009)^{*}$ \\
\hline Aro145 & $\operatorname{TrGC}$ & $\mathrm{PXYFUOOH}+\mathrm{OH} \rightarrow$ PXYFUO2 & $2.78 \mathrm{E}-11$ & Rickard and Pascoe (2009) \\
\hline Aro146 & $\operatorname{TrGCN}$ & $\mathrm{MC} 3 \mathrm{CODBPAN}+\mathrm{OH} \rightarrow \mathrm{GLYOX}+\mathrm{HCHO}+\mathrm{CO}+\mathrm{NO}_{2}$ & $4.37 \mathrm{E}-11$ & $\overline{\text { Rickard and Pascoe }}(\overline{\overline{2009}})$ \\
\hline Aro147 & TrGCN & $\mathrm{MC} 3 \mathrm{CODBPAN} \rightarrow \mathrm{MC} 3 \mathrm{CODBCO} 3+\mathrm{NO}_{2}$ & k_PAN_M & Rickard and Pascoe $(\overline{\overline{2009}})^{*}$ \\
\hline Aro148 & $\operatorname{TrGC}$ & $\begin{array}{l}\mathrm{C} 5 \mathrm{CO} 14 \mathrm{OH}+\mathrm{OH} \rightarrow .83 \mathrm{MALANHY}+.83 \mathrm{CH}_{3} \mathrm{O}_{2}+.17 \\
\text { MGLYOX }+.17 \mathrm{HO}_{2}+.17 \mathrm{CO}\end{array}$ & $5.44 \mathrm{E}-11$ & $\overline{\text { Rickard and Pascoe }}(\overline{\overline{2009}})^{*}$ \\
\hline Aro149 & $\operatorname{TrGC}$ & $\mathrm{NPXYFUO} 2+\mathrm{HO}_{2} \rightarrow \mathrm{NPXYFUOOH}$ & KRO2HO2 $* 0.706 *\left(1-r c o c h 2 \circ 2 \_o h\right)$ & Rickard and Pascoe (2009) \\
\hline Aro150 & TrGCN & $\mathrm{NPXYFUO} 2+\mathrm{HO}_{2} \rightarrow \mathrm{C} 23 \mathrm{O} 3 \mathrm{CCHO}+\mathrm{NO}_{2}+\mathrm{OH}$ & KRO2HO $2 * 0.706 *$ rcoch $2 \circ 2$ oh & $\overline{\text { Rickard and Pascoe }}(\overline{2009})^{*}$ \\
\hline Aro151 & $\operatorname{TrGCN}$ & $\mathrm{NPXYFUO} 2+\mathrm{NO} \rightarrow \mathrm{C} 23 \mathrm{O} 3 \mathrm{CCHO}+\mathrm{NO}_{2}+\mathrm{NO}_{2}$ & KRO2NO & $\overline{\text { Rickard and Pascoe }}(\overline{\overline{2009}})^{*}$ \\
\hline Aro152 & $\operatorname{TrGCN}$ & $\mathrm{NPXYFUO} 2+\mathrm{NO}_{3} \rightarrow \mathrm{C} 23 \mathrm{O} 3 \mathrm{CCHO}+\mathrm{NO}_{2}+\mathrm{NO}_{2}$ & KR02NO3 & $\overline{\text { Rickard and Pascoe }}(\overline{\overline{2009}})^{*}$ \\
\hline Aro153 & $\operatorname{TrGCN}$ & $\mathrm{NPXYFUO} 2 \rightarrow \mathrm{C} 23 \mathrm{O} 3 \mathrm{CCHO}+\mathrm{NO}_{2}$ & KR02tORO2*RO2 & $\overline{\text { Rickard and Pascoe }}(\overline{\overline{2009}})^{*}$ \\
\hline Aro154 & TrGCN & $\mathrm{C} 5 \mathrm{DICARB}+\mathrm{NO}_{3} \rightarrow \mathrm{C} 5 \mathrm{CO} 14 \mathrm{O} 2+\mathrm{HNO}_{3}$ & $\mathrm{KNO} O \mathrm{AL} * 2.75$ & Rickard and Pascoe (2009) \\
\hline Aro155 & TrGC & $\begin{array}{l}\text { C5DICARB }+\mathrm{O}_{3} \rightarrow .5338 \mathrm{GLYOX}+.063 \mathrm{CH}_{3} \mathrm{CHO}+ \\
.348 \mathrm{CH}_{3} \mathrm{C}(\mathrm{O}) \mathrm{OO}+.918 \mathrm{CO}+.57 \mathrm{OH}+.473 \mathrm{HO}_{2}+ \\
.0563 \mathrm{CH} 3 \mathrm{COCO} 2 \mathrm{H}+.5338 \mathrm{MGLYOX}+.676 \mathrm{H}_{2} \mathrm{O}_{2}+.063 \\
\mathrm{HCHO}+.0563 \mathrm{HCOCO}_{2} \mathrm{H}\end{array}$ & $2.00 \mathrm{E}-18$ & Rickard and Pascoe $(\overline{2009})$ \\
\hline Aro156 & TrGC & $\mathrm{C} 5 \mathrm{DICARB}+\mathrm{OH} \rightarrow .48 \mathrm{C} 5 \mathrm{CO} 14 \mathrm{O} 2+.52 \mathrm{C} 5 \mathrm{DICARBO} 2$ & $6.2 \mathrm{E}-11$ & Rickard and Pascoe (2009) \\
\hline Aro157 & TrGC & $\begin{array}{l}\mathrm{MC} 3 \mathrm{ODBCO} 2 \mathrm{H}+\mathrm{OH} \rightarrow .35 \mathrm{GLYOX}+.35 \mathrm{CH}_{3} \mathrm{O}_{2}+.35 \\
\mathrm{CO}+.65 \text { MMALANHY }+.65 \mathrm{HO}_{2}\end{array}$ & $4.38 \mathrm{E}-11$ & $\overline{\text { Rickard and Pascoe }}(\overline{2009})^{*}$ \\
\hline Aro158 & TrGC & $\mathrm{C} 23 \mathrm{O} 3 \mathrm{CCO} 2 \mathrm{H}+\mathrm{OH} \rightarrow \mathrm{MCOCOMOXO} 2$ & $8.76 \mathrm{E}-13$ & Rickard and Pascoe (2009) \\
\hline Aro159 & TrGC & $\mathrm{C} 23 \mathrm{O} 3 \mathrm{CCO} 3+\mathrm{HO}_{2} \rightarrow \mathrm{C} 23 \mathrm{O} 3 \mathrm{CCO} 2 \mathrm{H}+\mathrm{O}_{3}$ & KAPHO2*rco3_o3 & Rickard and Pascoe $(\overline{2009})^{*}$ \\
\hline Aro160 & TrGC & $\mathrm{C} 23 \mathrm{O} 3 \mathrm{CCO} 3+\mathrm{HO}_{2} \rightarrow \mathrm{C} 23 \mathrm{O} 3 \mathrm{CCO} 3 \mathrm{H}$ & KAPHO2*rco3_ooh & Rickard and Pascoe $(\overline{2009})^{*}$ \\
\hline Aro161 & $\operatorname{TrGC}$ & $\mathrm{C} 23 \mathrm{O} 3 \mathrm{CCO} 3+\mathrm{HO}_{2} \rightarrow \mathrm{MCOCOMOXO} 2+\mathrm{OH}$ & KAPHO2*rco3_oh & $\overline{\text { Rickard and Pascoe }}(\overline{\overline{2009}})^{*}$ \\
\hline
\end{tabular}


Table 1: Gas phase reactions (... continued)

\begin{tabular}{|c|c|c|c|c|}
\hline \# & labels & chemical reaction & rate coefficient & reference \\
\hline Aro162 & TrGCN & $\mathrm{C} 23 \mathrm{O} 3 \mathrm{CCO} 3+\mathrm{NO} \rightarrow \mathrm{MCOCOMOXO} 2+\mathrm{NO}_{2}$ & KAPNO & Rickard and Pascoe $(2009)$ \\
\hline Aro163 & TrGCN & $\mathrm{C} 23 \mathrm{O} 3 \mathrm{CCO} 3+\mathrm{NO}_{2} \rightarrow \mathrm{C} 23 \mathrm{O} 3 \mathrm{CPAN}$ & k_CH3CO3_NO2 & $\overline{\text { Rickard and Pascoe }}(\overline{\overline{2009}})^{*}$ \\
\hline Aro164 & $\operatorname{TrGCN}$ & $\mathrm{C} 23 \mathrm{O} 3 \mathrm{CCO} 3+\mathrm{NO}_{3} \rightarrow \mathrm{MCOCOMOXO} 2+\mathrm{NO}_{2}$ & KR02NO3*1.74 & $\overline{\text { Rickard and Pascoe }}(\overline{\overline{2009}})$ \\
\hline Aro165 & $\operatorname{TrGC}$ & $\mathrm{C} 23 \mathrm{O} 3 \mathrm{CCO} 3 \rightarrow \mathrm{MCOCOMOXO} 2$ & $\mathrm{KRO} 2 \mathrm{AP} * \mathrm{RO} 2$ & $\overline{\text { Rickard and Pascoe }}(\overline{\overline{2009}})^{*}$ \\
\hline Aro166 & $\operatorname{TrGCN}$ & TLFUONE $+\mathrm{NO}_{3} \rightarrow$ NTLFUO2 & $1.00 \mathrm{E}-12$ & $\overline{\text { Rickard and Pascoe }(\overline{2009})}$ \\
\hline Aro167 & $\operatorname{TrGC}$ & $\begin{array}{l}\text { TLFUONE }+\mathrm{O}_{3} \rightarrow .5 \mathrm{CO}+.5 \mathrm{OH}+.5 \text { MECOACETO } 2 \\
+.3125 \mathrm{C} 24 \mathrm{O} 3 \mathrm{CCO} 2 \mathrm{H}+.1875 \text { ACCOMECHO }+.1875 \\
\mathrm{H}_{2} \mathrm{O}_{2}\end{array}$ & $8.00 \mathrm{E}-19$ & $\overline{\overline{\text { Rickard and Pascoe }}}(\overline{\overline{2009}})^{*}$ \\
\hline Aro168 & TrGC & TLFUONE + OH $\rightarrow$ TLFUO2 & $6.90 \mathrm{E}-11$ & Rickard and Pascoe (2009) \\
\hline Aro169 & TrGC & $\mathrm{ACCOMECO} 3+\mathrm{HO}_{2} \rightarrow \mathrm{ACCOMECO} 3 \mathrm{H}$ & KAPHO2 $*\left(r c o 3 \_o o h+r c o 3 \_o 3\right)$ & Rickard and Pascoe $(2009)^{*}$ \\
\hline Aro170 & $\operatorname{TrGC}$ & $\mathrm{ACCOMECO} 3+\mathrm{HO}_{2} \rightarrow \mathrm{MECOACETO} 2+\mathrm{OH}$ & KAPHO2*rco3_oh & $\overline{\text { Rickard and Pascoe }}(\overline{\overline{2009}})^{*}$ \\
\hline Aro171 & $\operatorname{TrGCN}$ & $\mathrm{ACCOMECO} 3+\mathrm{NO} \rightarrow \mathrm{MECOACETO} 2+\mathrm{NO}_{2}$ & KAPNO & Rickard and Pascoe $(\overline{\overline{2009}})$ \\
\hline Aro172 & TrGCN & ACCOMECO $3+\mathrm{NO}_{2} \rightarrow$ ACCOMEPAN & k_CH3CO3_NO2 & Rickard and Pascoe $(\overline{2009})^{*}$ \\
\hline Aro173 & $\operatorname{TrGCN}$ & $\mathrm{ACCOMECO} 3+\mathrm{NO}_{3} \rightarrow \mathrm{MECOACETO} 2+\mathrm{NO}_{2}$ & KR02N03*1.74 & $\overline{\text { Rickard and Pascoe }(\overline{2009})}$ \\
\hline Aro174 & TrGC & ACCOMECO3 $\rightarrow$ MECOACETO 2 & $\mathrm{KRO} 2 \mathrm{AP} * \mathrm{RO} 2$ & $\overline{\overline{\text { Rickard and Pascoe }}}(\overline{\overline{2009}})$ \\
\hline Aro175 & TrGC & $\mathrm{C} 4 \mathrm{CO} 2 \mathrm{DCO} 3 \mathrm{H}+\mathrm{OH} \rightarrow \mathrm{C} 4 \mathrm{CO} 2 \mathrm{DBCO} 3$ & $3.06 \mathrm{E}-11$ & Rickard and Pascoe $(\overline{\overline{2009}})$ \\
\hline Aro176 & TrGCN & $\mathrm{EPXDLPAN}+\mathrm{OH} \rightarrow \mathrm{C} 33 \mathrm{CO}+\mathrm{CO}+\mathrm{NO}_{2}$ & $2.29 \mathrm{E}-11$ & Rickard and Pascoe $(\overline{2009})$ \\
\hline Aro177 & TrGCN & EPXDLPAN $\rightarrow$ EPXDLCO3 $+\mathrm{NO}_{2}$ & k_PAN_M & Rickard and Pascoe $(\overline{2009})^{*}$ \\
\hline Aro178 & $\operatorname{TrGC}$ & $\mathrm{C} 4 \mathrm{M} 2 \mathrm{ALOHO} 2+\mathrm{HO}_{2} \rightarrow \mathrm{C} 4 \mathrm{MALOHOOH}$ & $\begin{array}{l}\text { KRO2H02*0.706*(1-rcoch2o2 } \\
\text { oh-rchohch2o2_oh) }\end{array}$ & $\overline{\text { Rickard and Pascoe }}(\overline{\overline{2009}})$ \\
\hline Aro179 & $\operatorname{TrGC}$ & $\begin{array}{l}\mathrm{C} 4 \mathrm{M} 2 \mathrm{ALOHO} 2+\mathrm{HO}_{2} \rightarrow \mathrm{GLYOX}+\mathrm{MGLYOX}+\mathrm{HO}_{2}+ \\
\mathrm{OH}\end{array}$ & $\begin{array}{l}\text { KR02HO2*0.706*rcoch2o2_oh+rchohch } 22_{-} \\
\text {oh }\end{array}$ & Rickard and Pascoe $(2009)^{*}$ \\
\hline Aro180 & TrGCN & $\begin{array}{l}\mathrm{C} 4 \mathrm{M} 2 \mathrm{ALOHO} 2+\mathrm{NO} \rightarrow \mathrm{GLYOX}+\mathrm{MGLYOX}+\mathrm{HO}_{2}+ \\
\mathrm{NO}_{2}\end{array}$ & KRO2NO & Rickard and Pascoe $(2009)^{*}$ \\
\hline Aro181 & $\operatorname{TrGCN}$ & $\begin{array}{l}\mathrm{C} 4 \mathrm{M} 2 \mathrm{ALOHO} 2+\mathrm{NO}_{3} \rightarrow \mathrm{GLYOX}+\mathrm{MGLYOX}+\mathrm{HO}_{2}+ \\
\mathrm{NO}_{2}\end{array}$ & KRO2NO3 & Rickard and Pascoe $(2009)^{*}$ \\
\hline Aro182 & $\operatorname{TrGC}$ & $\mathrm{C} 4 \mathrm{M} 2 \mathrm{ALOHO} 2 \rightarrow \mathrm{GLYOX}+\mathrm{MGLYOX}+\mathrm{HO}_{2}$ & KRO2tORO2*RO2 & Rickard and Pascoe $(2009)^{*}$ \\
\hline Aro183 & $\operatorname{TrGCN}$ & $\mathrm{ACCOMECHO}+\mathrm{NO}_{3} \rightarrow \mathrm{ACCOMECO} 3+\mathrm{HNO}_{3}$ & KNO3AL $* 5.5$ & Rickard and Pascoe $(\overline{2009})$ \\
\hline Aro184 & TrGC & $\mathrm{ACCOMECHO}+\mathrm{OH} \rightarrow$ ACCOMECO 3 & $7.09 \mathrm{E}-11$ & $\overline{\overline{\text { Rickard and Pascoe }}}(\overline{\overline{2009}})$ \\
\hline Aro185 & TrGC & MMALNHYOOH $+\mathrm{OH} \rightarrow$ MMALANHYO2 & $1.69 \mathrm{E}-11$ & Rickard and Pascoe $(\overline{2009})$ \\
\hline Aro186 & TrGC & $\mathrm{C} 5 \mathrm{DICAROOH}+\mathrm{OH} \rightarrow \mathrm{C} 5134 \mathrm{CO} 2 \mathrm{OH}+\mathrm{OH}$ & $1.21 \mathrm{E}-10$ & Rickard and Pascoe $(\overline{2009})$ \\
\hline Aro187 & TrGC & $\mathrm{C} 5 \mathrm{DICAROOH}+\mathrm{OH} \rightarrow \mathrm{C} 5 \mathrm{DICARBO} 2$ & 1.90E-12*EXP (190/TEMP) & Rickard and Pascoe $(\overline{2009})$ \\
\hline Aro188 & $\operatorname{TrGC}$ & $\mathrm{C} 24 \mathrm{O} 3 \mathrm{CCO} 2 \mathrm{H}+\mathrm{OH} \rightarrow \mathrm{MECOACETO} 2$ & $8.76 \mathrm{E}-13$ & $\overline{\overline{\text { Rickard and Pascoe }}}(\overline{\overline{2009}})$ \\
\hline Aro189 & TrGCN & $\mathrm{NTLFUOOH}+\mathrm{OH} \rightarrow \mathrm{NTLFUO} 2$ & $4.44 \mathrm{E}-12$ & Rickard and Pascoe \\
\hline Aro190 & TrGC & $\mathrm{MECOACEOOH}+\mathrm{OH} \rightarrow$ MECOACETO2 & $3.59 \mathrm{E}-12$ & Rickard and Pascoe $(2009)$ \\
\hline
\end{tabular}


Table 1: Gas phase reactions (... continued)

\begin{tabular}{|c|c|c|c|c|}
\hline$\#$ & labels & chemical reaction & rate coefficient & reference \\
\hline Aro191 & TrGCN & $\begin{array}{l}\text { ACCOMEPAN }+\mathrm{OH} \rightarrow \text { METACETHO }+\mathrm{CO}+\mathrm{CO}+ \\
\mathrm{NO}_{2}\end{array}$ & $1.00 \mathrm{E}-14$ & $\overline{\text { Rickard and Pascoe }}(\overline{2009})$ \\
\hline Aro192 & TrGCN & $\mathrm{ACCOMEPAN} \rightarrow \mathrm{ACCOMECO} 3+\mathrm{NO}_{2}$ & k_PAN_M & Rickard and Pascoe $(2009)^{*}$ \\
\hline Aro193 & TrGC & $\mathrm{C} 4 \mathrm{MALOHOOH}+\mathrm{OH} \rightarrow$ GLYOX $+\mathrm{MGLYOX}+\mathrm{HO}_{2}$ & $4.58 \mathrm{E}-11$ & Rickard and Pascoe (2009)* \\
\hline Aro194 & $\operatorname{TrGC}$ & $\begin{array}{l}\mathrm{C} 3 \mathrm{MCODBCO} 3+\mathrm{HO}_{2} \rightarrow .35 \mathrm{MGLYOX}+.35 \mathrm{HO}_{2}+.35 \\
\mathrm{CO}+.65 \text { MMALANHY }+.65 \mathrm{HO}_{2}+\mathrm{OH}\end{array}$ & KAPHO2*rco3_oh & $\overline{\text { Rickard and Pascoe }}(\overline{2009})^{*}$ \\
\hline Aro195 & TrGC & $\mathrm{C} 3 \mathrm{MCODBCO} 3+\mathrm{HO}_{2} \rightarrow \mathrm{C} 4 \mathrm{CODBCO} 3 \mathrm{H}$ & $\mathrm{KAPHO} 2 *(r \mathrm{co}$ _ooh+rco3_o3 $)$ & Rickard and Pascoe (2009)* \\
\hline Aro196 & TrGCN & $\begin{array}{l}\mathrm{C} 3 \mathrm{MCODBCO} 3+\mathrm{NO} \rightarrow .35 \mathrm{MGLYOX}+.35 \mathrm{HO}_{2}+.35 \\
\mathrm{CO}+.65 \text { MMALANHY }+.65 \mathrm{HO}_{2}+\mathrm{NO}_{2}\end{array}$ & KAPNO & Rickard and Pascoe $(2009) *$ \\
\hline Aro197 & TrGCN & $\mathrm{C} 3 \mathrm{MCODBCO} 3+\mathrm{NO}_{2} \rightarrow$ C3MCODBPANN & k_CH3CO3_NO2 & Rickard and Pascoe (2009)* \\
\hline Aro198 & $\operatorname{TrGCN}$ & $\begin{array}{l}\mathrm{C} 3 \mathrm{MCODBCO} 3+\mathrm{NO}_{3} \rightarrow \mathrm{NO}_{2}+.35 \mathrm{MGLYOX}+.35 \\
\mathrm{HO}_{2}+.35 \mathrm{CO}+.65 \text { MMALANHY }+.65 \mathrm{HO}_{2}\end{array}$ & $\mathrm{KR} 02 \mathrm{~N} 03 * 1.74$ & Rickard and Pascoe $(\overline{2009})^{*}$ \\
\hline Aro199 & TrGC & $\begin{array}{l}\text { C3MCODBCO } 3 \rightarrow .35 \mathrm{MGLYOX}+.35 \mathrm{HO}_{2}+.35 \mathrm{CO}+ \\
.65 \text { MMALANHY }+.65 \mathrm{HO}_{2}\end{array}$ & $\mathrm{KR} 02 \mathrm{AP} * \mathrm{RO} 2$ & Rickard and Pascoe (2009)* \\
\hline Aro200 & TrGCN & PXYFUONE $+\mathrm{NO}_{3} \rightarrow$ NPXYFUO2 & $1.00 \mathrm{E}-12$ & Rickard and Pascoe 2009) \\
\hline Aro201 & TrGC & $\mathrm{PXYFUONE}+\mathrm{O}_{3} \rightarrow \mathrm{OH}+\mathrm{CO}+\mathrm{MCOCOMOXO} 2$ & $8.00 \mathrm{E}-19$ & Rickard and Pascoe $(\overline{\overline{2009}})$ \\
\hline Aro202 & TrGC & $\mathrm{PXYFUONE}+\mathrm{OH} \rightarrow \mathrm{PXYFUO2}$ & $2.42 \mathrm{E}-11$ & Rickard and Pascoe $(\overline{2009})$ \\
\hline Aro203 & $\operatorname{TrGC}$ & $\mathrm{TLFUO} 2+\mathrm{HO}_{2} \rightarrow$ TLFUOOH & $\begin{array}{l}\text { KRO2HO2*0.706* }\left(1-\mathrm{rcoch}_{2} \mathrm{o} 2_{-}\right. \\
\text {oh-rchohch2o2_oh) }\end{array}$ & Rickard and Pascoe $(\overline{2009})$ \\
\hline Aro204 & TrGC & TLFUO2 $+\mathrm{HO}_{2} \rightarrow \mathrm{ACCOMECHO}+\mathrm{HO}_{2}+\mathrm{OH}$ & $\begin{array}{l}\text { KRO2HO2 } * 0.706 *(\text { rcoch2o2_oh+ } \\
\text { rchohch2o2_oh })\end{array}$ & Rickard and Pascoe $(2009)^{*}$ \\
\hline Aro205 & TrGCN & TLFUO2 $+\mathrm{NO} \rightarrow$ ACCOMECHO $+\mathrm{HO}_{2}+\mathrm{NO}_{2}$ & KRO2NO & Rickard and Pascoe (2009)* \\
\hline Aro206 & $\operatorname{TrGCN}$ & $\mathrm{TLFUO} 2+\mathrm{NO}_{3} \rightarrow \mathrm{ACCOMECHO}+\mathrm{HO}_{2}+\mathrm{NO}_{2}$ & KRO2NO3 & $\overline{\text { Rickard and Pascoe }}(\overline{\overline{2009}})^{*}$ \\
\hline Aro207 & TrGC & TLFUO2 $\rightarrow$ ACCOMECHO $+\mathrm{HO}_{2}$ & KRO2tORO2*RO2 & Rickard and Pascoe $(\overline{2009})^{*}$ \\
\hline Aro208 & TrGC & $\mathrm{C} 5 \mathrm{CO} 14 \mathrm{OOH}+\mathrm{OH} \rightarrow \mathrm{C} 5 \mathrm{CO} 14 \mathrm{O} 2$ & $3.59 \mathrm{E}-12$ & Rickard and Pascoe (2009) \\
\hline Aro209 & TrGCN & $\mathrm{C} 23 \mathrm{O} 3 \mathrm{CPAN}+\mathrm{OH} \rightarrow \mathrm{CO}+\mathrm{C} 23 \mathrm{O} 3 \mathrm{CHO}+\mathrm{NO}_{2}$ & $7.36 \mathrm{E}-13$ & Rickard and Pascoe (2009) \\
\hline Aro210 & TrGCN & $\mathrm{C} 23 \mathrm{O} 3 \mathrm{CPAN} \rightarrow \mathrm{C} 23 \mathrm{O} 3 \mathrm{CCO} 3+\mathrm{NO}_{2}$ & k_PAN_M & Rickard and Pascoe (2009)* \\
\hline Aro211 & TrGC & $\mathrm{C} 4 \mathrm{CODBCO} 3 \mathrm{H}+\mathrm{OH} \rightarrow \mathrm{C} 3 \mathrm{MCODBCO} 3$ & $4.73 E-11$ & Rickard and Pascoe (2009) \\
\hline Aro212 & TrGC & $\mathrm{TLFUOOH}+\mathrm{OH} \rightarrow \mathrm{TLFUO} 2$ & $2.53 \mathrm{E}-11$ & Rickard and Pascoe (2009) \\
\hline Aro213 & $\operatorname{TrGC}$ & $\mathrm{C} 23 \mathrm{O} 3 \mathrm{CCO} 3 \mathrm{H}+\mathrm{OH} \rightarrow \mathrm{C} 23 \mathrm{O} 3 \mathrm{CCO} 3$ & $4.34 \mathrm{E}-12$ & Rickard and Pascoe $(\overline{2009})$ \\
\hline Aro214 & TrGC & $\mathrm{MC} 3 \mathrm{ODBCO} 3 \mathrm{H}+\mathrm{OH} \rightarrow \mathrm{MC} 3 \mathrm{CODBCO} 3$ & $4.73 E-11$ & Rickard and Pascoe $(\overline{2009})$ \\
\hline Aro215 & TrGC & $\mathrm{ACCOMECO} 3 \mathrm{H}+\mathrm{OH} \rightarrow \mathrm{ACCOMECO} 3$ & $3.59 \mathrm{E}-12$ & Rickard and Pascoe (2009) \\
\hline Aro216 & TrGC & C5DIALO $2+\mathrm{HO}_{2} \rightarrow$ C5DIALOOH & $\mathrm{KRO} 2 \mathrm{HO} 2 * 0.706 *\left(1-\mathrm{rcoch} 2 \mathrm{o} 2 \_\mathrm{oh}\right)$ & Rickard and Pascoe (2009) \\
\hline Aro217 & TrGC & $\mathrm{C} 5$ DIALO $2+\mathrm{HO}_{2} \rightarrow$ MALDIAL $+\mathrm{CO}+\mathrm{HO}_{2}+\mathrm{OH}$ & $\mathrm{KRO} 2 \mathrm{HO} 2 * 0.706 * \mathrm{rcoch} 2 \mathrm{O} 2$-oh & Rickard and Pascoe (2009)* \\
\hline Aro218 & TrGCN & C5DIALO2 $+\mathrm{NO} \rightarrow$ MALDIAL $+\mathrm{CO}+\mathrm{HO}_{2}+\mathrm{NO}_{2}$ & KRO2NO & Rickard and Pascoe (2009)* \\
\hline
\end{tabular}


Table 1: Gas phase reactions (... continued)

\begin{tabular}{|c|c|c|c|c|c|}
\hline \# & labels & chemical reaction & rate coefficient & reference & \\
\hline Aro219 & TrGCN & $\mathrm{C} 5$ DIALO $2+\mathrm{NO}_{3} \rightarrow$ MALDIAL $+\mathrm{CO}+\mathrm{HO}_{2}+\mathrm{NO}_{2}$ & KR02NO3 & Rickard and Pascoe & $(2009)^{*}$ \\
\hline Aro220 & TrGC & C5DIALO2 $\rightarrow$ MALDIAL $+\mathrm{CO}+\mathrm{HO}_{2}$ & $8.80 \mathrm{E}-13 * \mathrm{R} 02$ & Rickard and Pascoe & $(\overline{2009})^{*}$ \\
\hline Aro221 & TrGC & $\mathrm{PHENOOH}+\mathrm{OH} \rightarrow \mathrm{PHENO} 2$ & $1.16 \mathrm{E}-10$ & Rickard and Pascoe & $(2009)$ \\
\hline Aro222 & TrGC & $\mathrm{C} 6 \mathrm{CO} 4 \mathrm{DB}+\mathrm{OH} \rightarrow \mathrm{CO}+\mathrm{CO}+\mathrm{HO}_{2}+\mathrm{CO}+\mathrm{C} 33 \mathrm{CO}$ & $7.70 \mathrm{E}-11$ & Rickard and Pascoe & $(2009)$ \\
\hline Aro223 & TrGC & $\mathrm{C} 5 \mathrm{CO} 2 \mathrm{DCO} 3 \mathrm{H}+\mathrm{OH} \rightarrow \mathrm{C} 5 \mathrm{CO} 2 \mathrm{DBCO} 3$ & $3.60 \mathrm{E}-11$ & Rickard and Pascoe & $(\overline{2009})$ \\
\hline Aro224 & TrGCN & $\mathrm{NDNPHENOOH}+\mathrm{OH} \rightarrow \mathrm{NDNPHENO} 2$ & $1.90 \mathrm{E}-12 * \operatorname{EXP}(190 / \mathrm{TEMP})$ & Rickard and Pascoe & $(2009)$ \\
\hline Aro225 & TrGC & $\mathrm{C} 615 \mathrm{CO} 2 \mathrm{O} 2+\mathrm{HO}_{2} \rightarrow \mathrm{C} 615 \mathrm{CO} 2 \mathrm{OOH}$ & KRO2HO2 $* 0.770 *\left(1 .-r \operatorname{coch} 2 \mathrm{o} 2 \_\mathrm{oh}\right)$ & Rickard and Pascoe & $(\overline{2009})$ \\
\hline Aro226 & TrGC & $\mathrm{C} 615 \mathrm{CO} 2 \mathrm{O} 2+\mathrm{HO}_{2} \rightarrow \mathrm{C} 5 \mathrm{DICARB}+\mathrm{CO}+\mathrm{HO}_{2}+\mathrm{OH}$ & KRO2HO2*0.770*rcoch $2 \circ 2$-oh & Rickard and Pascoe & $(\overline{2009})^{*}$ \\
\hline Aro227 & TrGCN & $\mathrm{C} 615 \mathrm{CO} 2 \mathrm{O} 2+\mathrm{NO} \rightarrow \mathrm{C} 5 \mathrm{DICARB}+\mathrm{CO}+\mathrm{HO}_{2}+\mathrm{NO}_{2}$ & KRO2NO & Rickard and Pascoe & $(2009)^{*}$ \\
\hline Aro228 & TrGCN & $\mathrm{C} 615 \mathrm{CO} 2 \mathrm{O} 2+\mathrm{NO}_{3} \rightarrow \mathrm{C} 5 \mathrm{DICARB}+\mathrm{CO}+\mathrm{HO}_{2}+\mathrm{NO}_{2}$ & KR02N03 & Rickard and Pascoe & $(\overline{2009})^{*}$ \\
\hline Aro229 & TrGC & $\mathrm{C} 615 \mathrm{CO} 2 \mathrm{O} 2 \rightarrow \mathrm{C} 5 \mathrm{DICARB}+\mathrm{CO}+\mathrm{HO}_{2}$ & $8.80 \mathrm{E}-13 * \mathrm{R} 02$ & Rickard and Pascoe & $(2009)^{*}$ \\
\hline Aro230 & TrGCN & BZEMUCPAN $+\mathrm{OH} \rightarrow$ MALDIAL $+\mathrm{CO}+\mathrm{NO}_{2}$ & $4.05 \mathrm{E}-11$ & Rickard and Pascoe & $(\overline{2009})$ \\
\hline Aro231 & TrGCN & BZEMUCPAN $\rightarrow$ BZEMUCCO3 $+\mathrm{NO}_{2}$ & k_PAN_M & Rickard and Pascoe & $(2009)^{*}$ \\
\hline Aro232 & TrGCN & BZBIPERNO3 $+\mathrm{OH} \rightarrow$ BZOBIPEROH $+\mathrm{NO}_{2}$ & $7.30 \mathrm{E}-11$ & Rickard and Pascoe & $(2009)$ \\
\hline Aro233 & $\operatorname{TrGCN}$ & $\mathrm{HOC} 6 \mathrm{H} 4 \mathrm{NO} 2+\mathrm{NO}_{3} \rightarrow \mathrm{NPHEN} 1 \mathrm{O}+\mathrm{HNO}_{3}$ & $9.00 \mathrm{E}-14$ & Rickard and Pascoe & $(\overline{2009})$ \\
\hline Aro234 & TrGCN & $\mathrm{HOC} 6 \mathrm{H} 4 \mathrm{NO} 2+\mathrm{OH} \rightarrow \mathrm{NPHEN} 1 \mathrm{O}$ & $9.00 \mathrm{E}-13$ & Rickard and Pascoe & $(\overline{2009})$ \\
\hline Aro235 & TrGCN & NDNPHENO2 $+\mathrm{HO}_{2} \rightarrow$ NDNPHENOOH & KRO2HO2*0.770*(1-rchohch2o2_oh) & Rickard and Pascoe & $\overline{2009}$ \\
\hline Aro236 & TrGC & $\begin{array}{l}\mathrm{NDNPHENO} 2+\mathrm{HO}_{2} \rightarrow \mathrm{NC} 4 \mathrm{DCO} 2 \mathrm{H}+\mathrm{HNO}_{3}+\mathrm{CO}+ \\
\mathrm{CO}+\mathrm{NO}_{2}+\mathrm{OH}\end{array}$ & KRO2HO2*0.770*rchohch2o2_oh & Rickard and Pascoe & $(\overline{2009})^{*}$ \\
\hline Aro237 & TrGCN & $\begin{array}{l}\mathrm{NDNPHENO} 2+\mathrm{NO} \rightarrow \mathrm{NC} 4 \mathrm{DCO} 2 \mathrm{H}+\mathrm{HNO}_{3}+\mathrm{CO}+ \\
\mathrm{CO}+\mathrm{NO}_{2}+\mathrm{NO}_{2}\end{array}$ & KRO2NO & Rickard and Pascoe & $(2009)^{*}$ \\
\hline Aro238 & TrGCN & $\begin{array}{l}\mathrm{NDNPHENO} 2+\mathrm{NO}_{3} \rightarrow \mathrm{NC} 4 \mathrm{DCO} 2 \mathrm{H}+\mathrm{HNO}_{3}+\mathrm{CO}+ \\
\mathrm{CO}+\mathrm{NO}_{2}+\mathrm{NO}_{2}\end{array}$ & KRO2NO3 & Rickard and Pascoe & $(2009)^{*}$ \\
\hline Aro239 & TrGCN & $\begin{array}{l}\mathrm{NDNPHENO} 2 \rightarrow \mathrm{NC} 4 \mathrm{DCO} 2 \mathrm{H}+\mathrm{HNO}_{3}+\mathrm{CO}+\mathrm{CO}+ \\
\mathrm{NO}_{2}\end{array}$ & $8.00 \mathrm{E}-13 * \mathrm{R} 02$ & Rickard and Pascoe & $(2009)^{*}$ \\
\hline Aro240 & TrGC & $\mathrm{PBZQCO}+\mathrm{OH} \rightarrow \mathrm{C} 5 \mathrm{CO} 2 \mathrm{OHCO} 3$ & $6.07 \mathrm{E}-11$ & Rickard and Pascoe & $(2009)$ \\
\hline Aro241 & TrGCN & $\mathrm{CATECHOL}+\mathrm{NO}_{3} \rightarrow \mathrm{CATEC} 1 \mathrm{O}+\mathrm{HNO}_{3}$ & $9.9 \mathrm{E}-11$ & Rickard and Pascoe & $(2009)^{*}$ \\
\hline Aro242 & TrGC & $\begin{array}{l}\mathrm{CATECHOL}+\mathrm{O}_{3} \rightarrow \text { MALDALCO2H }+\mathrm{HCOCO}_{2} \mathrm{H}+ \\
\mathrm{HO}_{2}+\mathrm{OH}\end{array}$ & $9.2 \mathrm{E}-18$ & Rickard and Pascoe & $(\overline{2009})$ \\
\hline Aro243 & TrGC & CATECHOL + OH $\rightarrow$ CATEC1O & $1.0 \mathrm{E}-10$ & Rickard and Pascoe & $(2009)$ \\
\hline Aro244 & TrGC & $\mathrm{C} 5 \mathrm{COOHCO} 3 \mathrm{H}+\mathrm{OH} \rightarrow \mathrm{C} 5 \mathrm{CO} 2 \mathrm{OHCO} 3$ & $8.01 \mathrm{E}-11$ & Rickard and Pascoe & $(2009)$ \\
\hline Aro245 & TrGCN & $\mathrm{NCATECHOL}+\mathrm{NO}_{3} \rightarrow$ NNCATECO 2 & $2.60 \mathrm{E}-12$ & Rickard and Pascoe & $2009)$ \\
\hline Aro246 & TrGCN & NCATECHOL + OH $\rightarrow$ NCATECO 2 & $3.47 \mathrm{E}-12$ & Rickard and Pascoe & $(2009)$ \\
\hline Aro247 & TrGC & $\mathrm{C} 5 \mathrm{CO} 2 \mathrm{OHCO} 3+\mathrm{HO}_{2} \rightarrow \mathrm{C} 5 \mathrm{COOHCO} 3 \mathrm{H}$ & KAPHO $2 *\left(r c o 3 \_o o h+r c o 3 \_o 3\right)$ & Rickard and Pascoe & $(\overline{2009})^{*}$ \\
\hline
\end{tabular}


Table 1: Gas phase reactions (... continued)

\begin{tabular}{|c|c|c|c|c|}
\hline \# & labels & chemical reaction & rate coefficient & reference \\
\hline Aro248 & TrGC & $\begin{array}{l}\mathrm{C} 5 \mathrm{CO} 2 \mathrm{OHCO} 3+\mathrm{HO}_{2} \rightarrow \mathrm{HOCOC} 4 \mathrm{DIAL}+\mathrm{HO}_{2}+\mathrm{CO}+ \\
\mathrm{OH}\end{array}$ & KAPHO2*rco3_oh & Rickard and Pascoe $(\overline{2009})^{*}$ \\
\hline Aro249 & TrGCN & $\begin{array}{l}\mathrm{C} 5 \mathrm{CO} 2 \mathrm{OHCO} 3+\mathrm{NO} \rightarrow \mathrm{HOCOC} 4 \mathrm{DIAL}+\mathrm{HO}_{2}+\mathrm{CO}+ \\
\mathrm{NO}_{2}\end{array}$ & KAPNO & Rickard and Pascoe (2009) \\
\hline Aro250 & TrGCN & $\mathrm{C} 5 \mathrm{CO} 2 \mathrm{OHCO} 3+\mathrm{NO}_{2} \rightarrow \mathrm{C} 5 \mathrm{CO} 2 \mathrm{OHPAN}$ & $\mathrm{k}_{-} \mathrm{CH} 3 \mathrm{CO} 3 \_\mathrm{NO} 2$ & Rickard and Pascoe 2009$)^{*}$ \\
\hline Aro251 & TrGCN & $\begin{array}{l}\mathrm{C} 5 \mathrm{CO} 2 \mathrm{OHCO} 3+\mathrm{NO}_{3} \rightarrow \mathrm{HOCOC} 4 \mathrm{DIAL}+\mathrm{HO}_{2}+\mathrm{CO}+ \\
\mathrm{NO}_{2}\end{array}$ & KRO2NO3*1.74 & Rickard and Pascoe $(2009)$ \\
\hline Aro252 & TrGC & $\mathrm{C} 5 \mathrm{CO} 2 \mathrm{OHCO} 3 \rightarrow \mathrm{HOCOC} 4 \mathrm{DIAL}+\mathrm{HO}_{2}+\mathrm{CO}$ & $\mathrm{KRO} 2 \mathrm{AP} * \mathrm{RO} 2$ & Rickard and Pascoe (2009) \\
\hline Aro253 & TrGCN & BZEPOXMUC $+\mathrm{NO}_{3} \rightarrow$ BZEMUCCO $3+\mathrm{HNO}_{3}$ & $2 * \mathrm{KNO} 3 \mathrm{AL} * 2.75$ & Rickard and Pascoe $(\overline{2009})$ \\
\hline Aro254 & $\operatorname{TrGC}$ & $\begin{array}{l}\text { BZEPOXMUC }+\mathrm{O}_{3} \rightarrow \text { EPXC4DIAL }+.125 \mathrm{HCHO}+ \\
.1125 \mathrm{HCOCO}_{2} \mathrm{H}+.0675 \text { GLYOX }+.0675 \mathrm{H}_{2} \mathrm{O}_{2}+.82 \\
\mathrm{HO}_{2}+.57 \mathrm{OH}+1.265 \mathrm{CO}\end{array}$ & $2.00 \mathrm{E}-18$ & $\overline{\text { Rickard and Pascoe }}(\overline{\overline{2009}})^{*}$ \\
\hline Aro255 & $\operatorname{TrGC}$ & $\begin{array}{l}\text { BZEPOXMUC }+ \text { OH } \rightarrow .31 \text { BZEMUCCO3 }+.69 \\
\text { BZEMUCO2 }\end{array}$ & $6.08 \mathrm{E}-11$ & Rickard and Pascoe (2009) \\
\hline Aro256 & $\operatorname{TrGCN}$ & $\mathrm{NCATECO} 2+\mathrm{HO}_{2} \rightarrow \mathrm{NCATECOOH}$ & KRO2HO2*0.770*(1-rchohch2o2_oh) & Rickard and Pascoe (2009) \\
\hline Aro257 & TrGC & $\begin{array}{l}\mathrm{NCATECO} 2+\mathrm{HO}_{2} \rightarrow \mathrm{NC} 4 \mathrm{DCO} 2 \mathrm{H}+\mathrm{HCOCO}_{2} \mathrm{H}+\mathrm{HO}_{2} \\
+\mathrm{OH}\end{array}$ & KRO2HO2*0.770*rchohch2o2_oh & $\overline{\text { Rickard and Pascoe }}(\overline{\overline{2009}})^{*}$ \\
\hline Aro258 & TrGCN & $\begin{array}{l}\mathrm{NCATECO} 2+\mathrm{NO} \rightarrow \mathrm{NC} 4 \mathrm{DCO} 2 \mathrm{H}+\mathrm{HCOCO}_{2} \mathrm{H}+\mathrm{HO}_{2} \\
+\mathrm{NO}_{2}\end{array}$ & KRO2NO & Rickard and Pascoe $(2009)^{*}$ \\
\hline Aro259 & TrGCN & $\begin{array}{l}\mathrm{NCATECO} 2+\mathrm{NO}_{3} \rightarrow \mathrm{NC} 4 \mathrm{DCO} 2 \mathrm{H}+\mathrm{HCOCO}_{2} \mathrm{H}+\mathrm{HO}_{2} \\
+\mathrm{NO}_{2}\end{array}$ & KRO2NO3 & Rickard and Pascoe $(2009)^{*}$ \\
\hline Aro260 & TrGCN & $\mathrm{NCATECO} 2 \rightarrow \mathrm{NC} 4 \mathrm{DCO} 2 \mathrm{H}+\mathrm{HCOCO}_{2} \mathrm{H}+\mathrm{HO}_{2}$ & $8.00 \mathrm{E}-13 * \mathrm{R} 02$ & Rickard and Pascoe (2009)* \\
\hline Aro261 & TrGCN & $\mathrm{NPHEN1OOH}+\mathrm{OH} \rightarrow$ NPHEN1O2 & $9.00 \mathrm{E}-13$ & Rickard and Pascoe $(\overline{2009})$ \\
\hline Aro262 & TrGCN & $\mathrm{NPHENO} 2+\mathrm{HO}_{2} \rightarrow \mathrm{NPHENOOH}$ & KRO2HO2*0.770*(1-rchohch2o2_oh) & Rickard and Pascoe (2009) \\
\hline Aro263 & TrGCN & $\begin{array}{l}\mathrm{NPHENO} 2+\mathrm{HO}_{2} \rightarrow \mathrm{MALDALCO} 2 \mathrm{H}+\mathrm{GLYOX}+\mathrm{NO}_{2} \\
+\mathrm{OH}\end{array}$ & KRO2HO2*0.770*rchohch2o2_oh & $\overline{\text { Rickard and Pascoe }}(\overline{2009})^{*}$ \\
\hline Aro264 & TrGCN & $\begin{array}{l}\mathrm{NPHENO} 2+\mathrm{NO} \rightarrow \mathrm{MALDALCO} 2 \mathrm{H}+\mathrm{GLYOX}+\mathrm{NO}_{2} \\
+\mathrm{NO}_{2}\end{array}$ & KRO2NO & Rickard and Pascoe $(2009)^{*}$ \\
\hline Aro265 & TrGCN & $\begin{array}{l}\mathrm{NPHENO} 2+\mathrm{NO}_{3} \rightarrow \mathrm{MALDALCO} 2 \mathrm{H}+\mathrm{GLYOX}+\mathrm{NO}_{2} \\
+\mathrm{NO}_{2}\end{array}$ & KR02NO3 & Rickard and Pascoe $(2009)^{*}$ \\
\hline Aro266 & TrGCN & $\mathrm{NPHENO} 2 \rightarrow \mathrm{MALDALCO} 2 \mathrm{H}+\mathrm{GLYOX}+\mathrm{NO}_{2}$ & $8.00 \mathrm{E}-13 * \mathrm{R} 02$ & Rickard and Pascoe (2009)* \\
\hline Aro267 & TrGC & $\begin{array}{l}\text { BENZENE }+\mathrm{OH} \rightarrow .352 \text { BZBIPERO2 }+.118 \\
\text { BZEPOXMUC }+.118 \mathrm{HO}_{2}+.53 \text { PHENOL }+.53 \mathrm{HO}_{2}\end{array}$ & 2.3E-12*EXP $(-190 / \mathrm{TEMP})$ & Rickard and Pascoe $(\overline{2009})^{*}$ \\
\hline Aro268 & TrGCN & $\begin{array}{l}\mathrm{C} 5 \mathrm{CO} 2 \mathrm{OHPAN}+\mathrm{OH} \rightarrow \mathrm{HOCOC} 4 \mathrm{DIAL}+\mathrm{CO}+\mathrm{CO}+ \\
\mathrm{NO}_{2}\end{array}$ & $7.66 \mathrm{E}-11$ & Rickard and Pascoe (2009) \\
\hline
\end{tabular}


Table 1: Gas phase reactions (... continued)

\begin{tabular}{|c|c|c|c|c|}
\hline \# & labels & chemical reaction & rate coefficient & reference \\
\hline Aro269 & TrGCN & $\mathrm{C} 5 \mathrm{CO} 2 \mathrm{OHPAN} \rightarrow \mathrm{C} 5 \mathrm{CO} 2 \mathrm{OHCO} 3+\mathrm{NO}_{2}$ & k_PAN_M & Rickard and Pascoe 2009$)^{*}$ \\
\hline Aro270 & TrGCN & $\mathrm{CATEC} 1 \mathrm{O}+\mathrm{NO}_{2} \rightarrow \mathrm{NCATECHOL}$ & $2.08 \mathrm{E}-12$ & $\overline{\text { Rickard and Pascoe }}(\overline{\overline{2009}})$ \\
\hline Aro271 & TrGC & $\mathrm{CATEC} 1 \mathrm{O}+\mathrm{O}_{3} \rightarrow$ CATEC1O 2 & $2.86 \mathrm{E}-13$ & Rickard and Pascoe (2009) \\
\hline Aro272 & TrGC & BZEMUCCO + OH $\rightarrow$ EPXDLCO3 + GLYOX & $9.20 \mathrm{E}-11$ & Rickard and Pascoe $(\overline{2009})$ \\
\hline Aro273 & TrGCN & $\mathrm{NNCATECO} 2+\mathrm{HO}_{2} \rightarrow$ NNCATECOOH & KRO2HO2*0.770*(1-rchohch2o2_oh) & Rickard and Pascoe 2009) \\
\hline Aro274 & TrGCN & $\begin{array}{l}\mathrm{NNCATECO} 2+\mathrm{HO}_{2} \rightarrow \mathrm{NC} 4 \mathrm{DCO} 2 \mathrm{H}+\mathrm{HCOCO}_{2} \mathrm{H}+ \\
\mathrm{NO}_{2}+\mathrm{OH}\end{array}$ & KRO2HO2*0.770*(rchohch2o2_oh) & Rickard and Pascoe $(\overline{2009})^{*}$ \\
\hline Aro275 & TrGCN & $\begin{array}{l}\mathrm{NNCATECO} 2+\mathrm{NO} \rightarrow \mathrm{NC} 4 \mathrm{DCO} 2 \mathrm{H}+\mathrm{HCOCO}_{2} \mathrm{H}+\mathrm{NO}_{2} \\
+\mathrm{NO}_{2}\end{array}$ & KRO2NO & Rickard and Pascoe (2009)* \\
\hline Aro276 & TrGCN & $\begin{array}{l}\mathrm{NNCATECO} 2+\mathrm{NO}_{3} \rightarrow \mathrm{NC} 4 \mathrm{DCO} 2 \mathrm{H}+\mathrm{HCOCO}_{2} \mathrm{H}+ \\
\mathrm{NO}_{2}+\mathrm{NO}_{2}\end{array}$ & KRO2NO3 & Rickard and Pascoe $(2009)^{*}$ \\
\hline Aro277 & TrGCN & $\mathrm{NNCATECO} 2 \rightarrow \mathrm{NC} 4 \mathrm{DCO} 2 \mathrm{H}+\mathrm{HCOCO}_{2} \mathrm{H}+\mathrm{NO}_{2}$ & $8.00 \mathrm{E}-13 * \mathrm{R} 02$ & Rickard and Pascoe (2009)* \\
\hline Aro278 & TrGC & BZEMUCCO2H $+\mathrm{OH} \rightarrow$ C5DIALO2 & $4.06 \mathrm{E}-11$ & Rickard and Pascoe (2009) \\
\hline Aro279 & TrGCN & $\mathrm{NNCATECOOH}+\mathrm{OH} \rightarrow$ NNCATECO 2 & $1.90 \mathrm{E}-12 * \operatorname{EXP}(190 / \mathrm{TEMP})$ & Rickard and Pascoe (2009) \\
\hline Aro280 & TrGCN & $\mathrm{NPHEN} 1 \mathrm{O}+\mathrm{NO}_{2} \rightarrow$ DNPHEN & $2.08 \mathrm{E}-12$ & Rickard and Pascoe (2009) \\
\hline Aro281 & TrGCN & $\mathrm{NPHEN1O}+\mathrm{O}_{3} \rightarrow \mathrm{NPHEN1O} 2$ & $2.86 \mathrm{E}-13$ & Rickard and Pascoe $(\overline{\overline{2009}})$ \\
\hline Aro282 & TrGCN & DNPHEN $+\mathrm{NO}_{3} \rightarrow$ NDNPHENO2 & $2.25 \mathrm{E}-15$ & Rickard and Pascoe $(\overline{2009})$ \\
\hline Aro283 & TrGCN & DNPHEN + OH $\rightarrow$ DNPHENO2 & $3.00 \mathrm{E}-14$ & Rickard and Pascoe (2009) \\
\hline Aro284 & TrGCN & $\begin{array}{l}\text { PHENOL }+\mathrm{NO}_{3} \rightarrow .742 \mathrm{C} 6 \mathrm{H} 5 \mathrm{O}+.742 \mathrm{HNO}_{3}+.258 \\
\text { NPHENO2 }\end{array}$ & $3.8 \mathrm{E}-12$ & Rickard and Pascoe $(\overline{2009})^{*}$ \\
\hline Aro285 & $\operatorname{TrGC}$ & $\begin{array}{l}\text { PHENOL }+ \text { OH } \rightarrow .06 \mathrm{C} 6 \mathrm{H} 5 \mathrm{O}+.8 \mathrm{CATECHOL}+.8 \mathrm{HO}_{2} \\
+.14 \mathrm{PHENO} 2\end{array}$ & 4.7E-13*EXP (1220/TEMP) & Rickard and Pascoe (2009)* \\
\hline Aro286 & $\operatorname{TrGCN}$ & $\mathrm{PBZQONE}+\mathrm{NO}_{3} \rightarrow \mathrm{NBZQO} 2$ & $3.00 \mathrm{E}-13$ & Rickard and Pascoe (2009) \\
\hline Aro287 & TrGC & $\mathrm{PBZQONE}+\mathrm{OH} \rightarrow \mathrm{PBZQO} 2$ & $4.6 \mathrm{E}-12$ & Rickard and Pascoe $(\overline{2009})$ \\
\hline Aro288 & TrGC & $\mathrm{PHENO} 2+\mathrm{HO}_{2} \rightarrow \mathrm{PHENOOH}$ & KRO2HO2*0.770*(1-rchohch2o2_oh) & Rickard and Pascoe \\
\hline Aro289 & TrGC & $\begin{array}{l}\text { PHENO2 }+\mathrm{HO}_{2} \rightarrow .71 \mathrm{MALDALCO} 2 \mathrm{H}+.71 \mathrm{GLYOX}+ \\
.29 \mathrm{PBZQONE}+\mathrm{HO}_{2}+\mathrm{OH}\end{array}$ & KRO2HO2*0.770*rchohch2o2_oh & Rickard and Pascoe (2009)* \\
\hline Aro290 & TrGCN & $\begin{array}{l}\text { PHENO2 }+\mathrm{NO} \rightarrow .71 \text { MALDALCO2H }+.71 \text { GLYOX }+ \\
.29 \text { PBZQONE }+\mathrm{HO}_{2}+\mathrm{NO}_{2}\end{array}$ & KRO2NO & Rickard and Pascoe (2009)* \\
\hline Aro291 & TrGCN & $\begin{array}{l}\text { PHENO2 }+\mathrm{NO}_{3} \rightarrow .71 \text { MALDALCO2H }+.71 \text { GLYOX }+ \\
.29 \text { PBZQONE }+\mathrm{HO}_{2}+\mathrm{NO}_{2}\end{array}$ & KR02NO3 & Rickard and Pascoe (2009)* \\
\hline Aro292 & TrGC & $\begin{array}{l}\text { PHENO2 } \rightarrow .71 \text { MALDALCO2H }+.71 \text { GLYOX }+.29 \\
\text { PBZQONE }+\mathrm{HO}_{2}\end{array}$ & $8.00 \mathrm{E}-13 * \mathrm{R} 02$ & Rickard and Pascoe (2009)* \\
\hline Aro293 & TrGC & $\mathrm{C} 615 \mathrm{CO} 2 \mathrm{OOH}+\mathrm{OH} \rightarrow \mathrm{C} 6125 \mathrm{CO}+\mathrm{OH}$ & $9.42 \mathrm{E}-11$ & Rickard and Pascoe (2009) \\
\hline Aro294 & TrGC & $\mathrm{C} 5 \mathrm{CO} 2 \mathrm{DBCO} 3+\mathrm{HO}_{2} \rightarrow \mathrm{C} 5 \mathrm{CO} 2 \mathrm{DCO} 3 \mathrm{H}$ & KAPHO2* $\left(r c o 3 \_o o h+r c 03 \_o 3\right)$ & Rickard and Pascoe (2009)* \\
\hline
\end{tabular}


Table 1: Gas phase reactions (... continued)

\begin{tabular}{|c|c|c|c|c|}
\hline \# & labels & chemical reaction & rate coefficient & reference \\
\hline Aro295 & TrGC & $\mathrm{C} 5 \mathrm{CO} 2 \mathrm{DBCO} 3+\mathrm{HO}_{2} \rightarrow \mathrm{CH}_{3} \mathrm{C}(\mathrm{O}) \mathrm{OO}+\mathrm{C} 33 \mathrm{CO}+\mathrm{OH}$ & KAPHO2*rco3_oh & Rickard and Pascoe $(2009)^{*}$ \\
\hline Aro296 & TrGCN & $\mathrm{C} 5 \mathrm{CO} 2 \mathrm{DBCO} 3+\mathrm{NO} \rightarrow \mathrm{CH}_{3} \mathrm{C}(\mathrm{O}) \mathrm{OO}+\mathrm{C} 33 \mathrm{CO}+\mathrm{NO}_{2}$ & KAPNO & Rickard and Pascoe (2009) \\
\hline Aro297 & $\operatorname{TrGCN}$ & $\mathrm{C} 5 \mathrm{CO} 2 \mathrm{DBCO} 3+\mathrm{NO}_{2} \rightarrow \mathrm{C} 5 \mathrm{CO} 2 \mathrm{DBPAN}$ & k_CH3CO3_NO2 & $\overline{\text { Rickard and Pascoe }}(\overline{\overline{2009}})^{*}$ \\
\hline Aro298 & TrGCN & $\mathrm{C} 5 \mathrm{CO} 2 \mathrm{DBCO} 3+\mathrm{NO}_{3} \rightarrow \mathrm{CH}_{3} \mathrm{C}(\mathrm{O}) \mathrm{OO}+\mathrm{C} 33 \mathrm{CO}+\mathrm{NO}_{2}$ & KR02N03*1.74 & Rickard and Pascoe $(\overline{2009})$ \\
\hline Aro299 & TrGC & $\mathrm{C} 5 \mathrm{CO} 2 \mathrm{DBCO} 3 \rightarrow \mathrm{CH}_{3} \mathrm{C}(\mathrm{O}) \mathrm{OO}+\mathrm{C} 33 \mathrm{CO}$ & $\mathrm{KRO} 2 \mathrm{AP} * \mathrm{RO} 2$ & Rickard and Pascoe $(\overline{2009})$ \\
\hline Aro300 & $\operatorname{TrGCN}$ & $\mathrm{NPHEN} 1 \mathrm{O} 2+\mathrm{HO}_{2} \rightarrow \mathrm{NPHEN1OOH}$ & $\mathrm{KRO} 2 \mathrm{HO} 2 * 0.770$ & $\overline{\text { Rickard and Pascoe }}(\overline{\overline{2009}})$ \\
\hline Aro301 & TrGCN & $\mathrm{NPHEN1O} 2+\mathrm{NO} \rightarrow \mathrm{NPHEN1O}+\mathrm{NO}_{2}$ & KRO2NO & $\overline{\text { Rickard and Pascoe }}(\overline{2009})$ \\
\hline Aro302 & TrGCN & $\mathrm{NPHEN} 1 \mathrm{O} 2+\mathrm{NO}_{2} \rightarrow \mathrm{NPHEN} 1 \mathrm{O}+\mathrm{NO}_{3}$ & KPHENO2NO2 & Jagiella and Zabel $(2007)^{*}$ \\
\hline Aro303 & TrGCN & $\mathrm{NPHEN} 1 \mathrm{O} 2+\mathrm{NO}_{3} \rightarrow \mathrm{NPHEN} 1 \mathrm{O}+\mathrm{NO}_{2}$ & KR02NO3 & Rickard and Pascoe 2009) \\
\hline Aro304 & $\operatorname{TrGCN}$ & NPHEN1O2 $\rightarrow$ NPHEN1O & KR02sR02*R02 & $\overline{\text { Rickard and Pascoe }}(\overline{\overline{2009}})$ \\
\hline Aro305 & TrGCN & $\mathrm{NPHENOOH}+\mathrm{OH} \rightarrow \mathrm{NPHENO} 2$ & $1.07 \mathrm{E}-10$ & $\overline{\text { Rickard and Pascoe }}(\overline{2009})$ \\
\hline Aro306 & $\operatorname{TrGCN}$ & $\mathrm{C} 6 \mathrm{H} 5 \mathrm{O}+\mathrm{NO}_{2} \rightarrow \mathrm{HOC} 6 \mathrm{H} 4 \mathrm{NO} 2$ & $2.08 \mathrm{E}-12$ & $\overline{\text { Rickard and Pascoe }}(\overline{\overline{2009}})^{*}$ \\
\hline Aro307 & TrGC & $\mathrm{C} 6 \mathrm{H} 5 \mathrm{O}+\mathrm{O}_{3} \rightarrow \mathrm{C} 6 \mathrm{H} 5 \mathrm{O} 2$ & $2.86 \mathrm{E}-13$ & Rickard and Pascoe $(\overline{\overline{2009}})$ \\
\hline Aro308 & TrGCN & $\mathrm{NCATECOOH}+\mathrm{OH} \rightarrow$ NCATECO 2 & 1.90E-12*EXP (190/TEMP) & Rickard and Pascoe $(\overline{\overline{2009}})$ \\
\hline Aro309 & TrGC & $\mathrm{PBZQOOH}+\mathrm{OH} \rightarrow \mathrm{PBZQCO}+\mathrm{OH}$ & $1.23 \mathrm{E}-10$ & Rickard and Pascoe $(\overline{2009})$ \\
\hline Aro310 & TrGC & $\mathrm{PBZQO} 2+\mathrm{HO}_{2} \rightarrow \mathrm{PBZQOOH}$ & $\begin{array}{l}\text { KRO2HO2*0.770* }\left(1-r c h o h c h 2 \circ 2_{-}\right. \\
\text {oh-rcoch2o2_oh) }\end{array}$ & Rickard and Pascoe $(\overline{2009})$ \\
\hline Aro311 & TrGC & $\mathrm{PBZQO} 2+\mathrm{HO}_{2} \rightarrow \mathrm{C} 5 \mathrm{CO} 2 \mathrm{OHCO} 3+\mathrm{OH}$ & $\begin{array}{l}\text { KRO2HO2*0.770*(rchohch2o2_oh+rcoch2o2 } \\
\text { oh) }\end{array}$ & Rickard and Pascoe $(2009) *$ \\
\hline Aro312 & $\operatorname{TrGCN}$ & $\mathrm{PBZQO} 2+\mathrm{NO} \rightarrow \mathrm{C} 5 \mathrm{CO} 2 \mathrm{OHCO} 3+\mathrm{NO}_{2}$ & KRO2NO & Rickard and Pascoe $(2009)^{*}$ \\
\hline Aro313 & TrGCN & $\mathrm{PBZQO} 2+\mathrm{NO}_{3} \rightarrow \mathrm{C} 5 \mathrm{CO} 2 \mathrm{OHCO} 3+\mathrm{NO}_{2}$ & KRO2NO3 & $\overline{\text { Rickard and Pascoe }}(\overline{\overline{2009}})^{*}$ \\
\hline Aro314 & TrGC & $\mathrm{PBZQO} 2 \rightarrow \mathrm{C} 5 \mathrm{CO} 2 \mathrm{OHCO} 3$ & $8.80 \mathrm{E}-13 * \mathrm{R} 02$ & Rickard and Pascoe $(\overline{2009})^{*}$ \\
\hline Aro315 & $\operatorname{TrGC}$ & BZOBIPEROH $+\mathrm{OH} \rightarrow$ MALDIALCO $3+$ GLYOX & $8.16 \mathrm{E}-11$ & $\overline{\text { Rickard and Pascoe }}(\overline{\overline{2009}})$ \\
\hline Aro316 & $\operatorname{TrGCN}$ & DNPHENO2 $+\mathrm{HO}_{2} \rightarrow$ DNPHENOOH & KRO2HO2*0.770*(1-rchohch2o2_oh) & Rickard and Pascoe $(\overline{2009})$ \\
\hline Aro317 & TrGCN & $\begin{array}{l}\mathrm{DNPHENO} 2+\mathrm{HO}_{2} \rightarrow \mathrm{NC} 4 \mathrm{DCO} 2 \mathrm{H}+\mathrm{HCOCO}_{2} \mathrm{H}+\mathrm{NO}_{2} \\
+\mathrm{OH}\end{array}$ & KRO2HO2*0.770*rchohch2o2_oh & Rickard and Pascoe $(\overline{\overline{2009}})^{*}$ \\
\hline Aro318 & TrGCN & $\begin{array}{l}\mathrm{DNPHENO} 2+\mathrm{NO} \rightarrow \mathrm{NC} 4 \mathrm{DCO} 2 \mathrm{H}+\mathrm{HCOCO}_{2} \mathrm{H}+\mathrm{NO}_{2} \\
+\mathrm{NO}_{2}\end{array}$ & KRO2NO & Rickard and Pascoe (2009)* \\
\hline Aro319 & TrGCN & $\begin{array}{l}\mathrm{DNPHENO} 2+\mathrm{NO}_{3} \rightarrow \mathrm{NC} 4 \mathrm{DCO} 2 \mathrm{H}+\mathrm{HCOCO}_{2} \mathrm{H}+\mathrm{NO}_{2} \\
+\mathrm{NO}_{2}\end{array}$ & KR02NO3 & Rickard and Pascoe $(2009)^{*}$ \\
\hline Aro320 & TrGCN & DNPHENO2 $\rightarrow$ NC4DCO2H $+\mathrm{HCOCO}_{2} \mathrm{H}+\mathrm{NO}_{2}$ & $8.00 \mathrm{E}-13 * \mathrm{R} 02$ & Rickard and Pascoe (2009)* \\
\hline Aro321 & TrGC & BZBIPEROOH + OH $\rightarrow$ BZOBIPEROH + OH & $9.77 \mathrm{E}-11$ & Rickard and Pascoe $(\overline{2009})$ \\
\hline Aro322 & $\operatorname{TrGC}$ & $\mathrm{BZEMUCO} 2+\mathrm{HO}_{2} \rightarrow$ BZEMUCOOH & $\begin{array}{l}\text { KRO2HO2*0.770* (1-rchohch } 2 \circ 2_{-} \\
\text {oh-rcoch2o2_oh) }\end{array}$ & $\overline{\bar{R} \text { Rickard and Pascoe }}(\overline{\overline{2009}})$ \\
\hline
\end{tabular}


Table 1: Gas phase reactions (... continued)

\begin{tabular}{|c|c|c|c|c|c|}
\hline \# & labels & chemical reaction & rate coefficient & reference & \\
\hline Aro323 & $\operatorname{TrGC}$ & $\begin{array}{l}\text { BZEMUCO } 2+\mathrm{HO}_{2} \rightarrow .5 \text { EPXC4DIAL }+.5 \mathrm{GLYOX}+.5 \\
\mathrm{HO}_{2}+.5 \mathrm{C} 3 \mathrm{DIALO} 2+.5 \mathrm{C} 32 \mathrm{OH} 13 \mathrm{CO}+\mathrm{OH}\end{array}$ & $\begin{array}{l}\text { KR02H02*0.770*(rchohch2o2_oh+rcoch2o2_ } \\
\text { oh) }\end{array}$ & Rickard and Pascoe & $(2009)^{*}$ \\
\hline Aro324 & $\operatorname{TrGCN}$ & $\mathrm{BZEMUCO} 2+\mathrm{NO} \rightarrow$ BZEMUCNO 3 & KRO2NO $* 0.105$ & Rickard and Pascoe & $(2009)$ \\
\hline Aro325 & TrGCN & $\begin{array}{l}\text { BZEMUCO } 2+\mathrm{NO} \rightarrow .5 \text { EPXC4DIAL }+.5 \text { GLYOX }+.5 \\
\mathrm{HO}_{2}+.5 \mathrm{C} 3 \mathrm{DIALO} 2+.5 \mathrm{C} 32 \mathrm{OH} 13 \mathrm{CO}+\mathrm{NO}_{2}\end{array}$ & KR02NO $* 0.895$ & Rickard and Pascoe & $(\overline{2009})^{*}$ \\
\hline Aro326 & $\operatorname{TrGCN}$ & $\begin{array}{l}\text { BZEMUCO } 2+\mathrm{NO}_{3} \rightarrow .5 \text { EPXC4DIAL }+.5 \mathrm{GLYOX}+.5 \\
\mathrm{HO}_{2}+.5 \mathrm{C} 3 \mathrm{DIALO} 2+.5 \mathrm{C} 32 \mathrm{OH} 13 \mathrm{CO}+\mathrm{NO}_{2}\end{array}$ & KRO2NO3 & Rickard and Pascoe & $(2009)^{*}$ \\
\hline Aro327 & $\operatorname{TrGC}$ & $\begin{array}{l}\text { BZEMUCO } 2 \rightarrow .5 \text { EPXC4DIAL }+.5 \text { GLYOX }+.5 \mathrm{HO}_{2}+ \\
.5 \mathrm{C} 3 \mathrm{DIALO} 2+.5 \mathrm{C} 32 \mathrm{OH} 13 \mathrm{CO}\end{array}$ & $8.80 \mathrm{E}-13 * \mathrm{R} 02$ & Rickard and Pascoe & $(2009)^{*}$ \\
\hline Aro328 & $\operatorname{TrGCN}$ & $\mathrm{C} 5 \mathrm{CO} 2 \mathrm{DBPAN}+\mathrm{OH} \rightarrow \mathrm{C} 33 \mathrm{CO}+\mathrm{CH}_{3} \mathrm{CHO}+\mathrm{NO}_{2}$ & $3.28 \mathrm{E}-11$ & Rickard and Pascoe & 2009 \\
\hline Aro329 & $\operatorname{TrGCN}$ & $\mathrm{C} 5 \mathrm{CO} 2 \mathrm{DBPAN} \rightarrow \mathrm{C} 5 \mathrm{CO} 2 \mathrm{DBCO} 3+\mathrm{NO}_{2}$ & k_PAN_M & Rickard and Pascoe & $(2009)^{*}$ \\
\hline Aro330 & $\operatorname{TrGCN}$ & $\mathrm{NBZQOOH}+\mathrm{OH} \rightarrow \mathrm{NBZQO} 2$ & $6.68 \mathrm{E}-11$ & Rickard and Pascoe & $(\overline{2009})$ \\
\hline Aro331 & TrGC & CATEC1OOH $+\mathrm{OH} \rightarrow$ CATEC1O2 & $1.90 \mathrm{E}-12 * \operatorname{EXP}(190 / \mathrm{TEMP})$ & Rickard and Pascoe & $(\overline{2009})$ \\
\hline Aro332 & $\operatorname{TrGC}$ & $\mathrm{C} 6125 \mathrm{CO}+\mathrm{OH} \rightarrow \mathrm{C} 5 \mathrm{CO} 14 \mathrm{O} 2+\mathrm{CO}$ & $6.45 \mathrm{E}-11$ & Rickard and Pascoe & $(2009)$ \\
\hline Aro333 & $\operatorname{TrGCN}$ & $\mathrm{NBZQO} 2+\mathrm{HO}_{2} \rightarrow \mathrm{NBZQOOH}$ & $\mathrm{KRO} 2 \mathrm{HO} 2 * 0.770 *\left(1-\mathrm{rcoch} 2 \mathrm{o} 2 \_\mathrm{oh}\right)$ & Rickard and Pascoe & $(2009)$ \\
\hline Aro334 & TrGCN & $\mathrm{NBZQO} 2+\mathrm{HO}_{2} \rightarrow \mathrm{C} 6 \mathrm{CO} 4 \mathrm{DB}+\mathrm{NO}_{2}+\mathrm{OH}$ & KRO2HO $2 * 0.770 *$ rcoch $2 \circ 2$ oh & Rickard and Pascoe & $(2009)^{*}$ \\
\hline Aro335 & TrGCN & $\mathrm{NBZQO} 2+\mathrm{NO} \rightarrow \mathrm{C} 6 \mathrm{CO} 4 \mathrm{DB}+\mathrm{NO}_{2}+\mathrm{NO}_{2}$ & KRO2NO & Rickard and Pascoe & $(2009)^{*}$ \\
\hline Aro336 & TrGCN & $\mathrm{NBZQO} 2+\mathrm{NO}_{3} \rightarrow \mathrm{C} 6 \mathrm{CO} 4 \mathrm{DB}+\mathrm{NO}_{2}+\mathrm{NO}_{2}$ & KR02NO3 & Rickard and Pascoe & $(\overline{2009})^{*}$ \\
\hline Aro337 & $\operatorname{TrGCN}$ & $\mathrm{NBZQO} 2 \rightarrow \mathrm{C} 6 \mathrm{CO} 4 \mathrm{DB}+\mathrm{NO}_{2}$ & $8.80 \mathrm{E}-13 * \mathrm{R} 02$ & Rickard and Pascoe & $(2009)^{*}$ \\
\hline Aro338 & TrGCN & DNPHENOOH $+\mathrm{OH} \rightarrow$ DNPHENO2 & $1.90 \mathrm{E}-12 * \operatorname{EXP}(190 / \mathrm{TEMP})$ & Rickard and Pascoe & $(\overline{2009})$ \\
\hline Aro339 & $\operatorname{TrGC}$ & $\mathrm{CATEC} 1 \mathrm{O} 2+\mathrm{HO}_{2} \rightarrow \mathrm{CATEC} 1 \mathrm{OOH}$ & $\mathrm{KRO} 2 \mathrm{HO} 2 * 0.770$ & Rickard and Pascoe & $(\overline{2009})$ \\
\hline Aro340 & $\operatorname{TrGCN}$ & $\mathrm{CATEC} 1 \mathrm{O} 2+\mathrm{NO} \rightarrow \mathrm{CATEC} 1 \mathrm{O}+\mathrm{NO}_{2}$ & KRO2NO & Rickard and Pascoe & $(2009)$ \\
\hline Aro341 & $\operatorname{TrGCN}$ & $\mathrm{CATEC} 1 \mathrm{O} 2+\mathrm{NO}_{2} \rightarrow \mathrm{CATEC} 1 \mathrm{O}+\mathrm{NO}_{3}$ & KPHENO2NO2 & Jagiella and Zabel & $(2007)^{*}$ \\
\hline Aro342 & TrGCN & $\mathrm{CATEC} 1 \mathrm{O} 2+\mathrm{NO}_{3} \rightarrow \mathrm{CATEC} 1 \mathrm{O}+\mathrm{NO}_{2}$ & KR02NO3 & Rickard and Pascoe & $(2009)$ \\
\hline Aro343 & $\operatorname{TrGC}$ & CATEC1O2 $\rightarrow$ CATEC1O & $8.80 \mathrm{E}-13 * \mathrm{R} 02$ & Rickard and Pascoe & $(\overline{2009})$ \\
\hline Aro344 & $\operatorname{TrGC}$ & BZEMUCCO3H $+\mathrm{OH} \rightarrow$ BZEMUCCO3 & $4.37 \mathrm{E}-11$ & Rickard and Pascoe & $(2009)$ \\
\hline Aro345 & TrGC & $\mathrm{C} 6 \mathrm{H} 5 \mathrm{OOH}+\mathrm{OH} \rightarrow \mathrm{C} 6 \mathrm{H} 5 \mathrm{O} 2$ & $3.60 \mathrm{E}-12$ & Rickard and Pascoe & $(\overline{2009})$ \\
\hline Aro346 & $\operatorname{TrGC}$ & $\mathrm{BZEMUCOOH}+\mathrm{OH} \rightarrow \mathrm{BZEMUCCO}+\mathrm{OH}$ & $1.31 \mathrm{E}-10$ & Rickard and Pascoe & $(\overline{2009})$ \\
\hline Aro347 & TrGC & $\mathrm{BZEMUCCO} 3+\mathrm{HO}_{2} \rightarrow \mathrm{BZEMUCCO} 2 \mathrm{H}+\mathrm{O}_{3}$ & KAPHO2*rco3_o3 & Rickard and Pascoe & $(2009)^{*}$ \\
\hline Aro348 & TrGC & BZEMUCCO $3+\mathrm{HO}_{2} \rightarrow$ BZEMUCCO3H & $\mathrm{KAPHO} 2 *$ rco3_ooh & Rickard and Pascoe & $2009)^{*}$ \\
\hline Aro349 & TrGC & $\mathrm{BZEMUCCO} 3+\mathrm{HO}_{2} \rightarrow \mathrm{C} 5 \mathrm{DIALO} 2+\mathrm{OH}$ & KAPHO2*rco3_oh & Rickard and Pascoe & $(2009)^{*}$ \\
\hline Aro350 & $\operatorname{TrGCN}$ & $\mathrm{BZEMUCCO} 3+\mathrm{NO} \rightarrow \mathrm{C} 5 \mathrm{DIALO} 2+\mathrm{NO}_{2}$ & KAPNO & Rickard and Pascoe & $\overline{2009}$ \\
\hline Aro351 & $\operatorname{TrGCN}$ & BZEMUCCO3 $+\mathrm{NO}_{2} \rightarrow$ BZEMUCPAN & k_CH3CO3_NO2 & Rickard and Pascoe & $(2009)^{*}$ \\
\hline Aro352 & TrGCN & BZEMUCCO3 $+\mathrm{NO}_{3} \rightarrow$ C5DIALO2 $+\mathrm{NO}_{2}$ & KR02N03*1.74 & Rickard and Pascoe & $(\overline{2009}$ \\
\hline Aro353 & $\operatorname{TrGC}$ & BZEMUCCO3 $\rightarrow$ C5DIALO2 & $1.00 \mathrm{E}-11 * \mathrm{R} 02$ & Rickard and Pascoe & $(2009)^{*}$ \\
\hline
\end{tabular}


Table 1: Gas phase reactions (... continued)

\begin{tabular}{|c|c|c|c|c|}
\hline$\#$ & labels & chemical reaction & rate coefficient & reference \\
\hline Aro354 & TrGC & $\mathrm{C} 6 \mathrm{H} 5 \mathrm{O} 2+\mathrm{HO}_{2} \rightarrow \mathrm{C} 6 \mathrm{H} 5 \mathrm{OOH}$ & KRO2HO2*0.770 & Rickard and Pascoe 2009) \\
\hline Aro355 & TrGCN & $\mathrm{C} 6 \mathrm{H} 5 \mathrm{O} 2+\mathrm{NO} \rightarrow \mathrm{C} 6 \mathrm{H} 5 \mathrm{O}+\mathrm{NO}_{2}$ & KRO2NO & Rickard and Pascoe (2009) \\
\hline Aro356 & TrGCN & $\mathrm{C} 6 \mathrm{H} 5 \mathrm{O} 2+\mathrm{NO}_{2} \rightarrow \mathrm{C} 6 \mathrm{H} 5 \mathrm{O}+\mathrm{NO}_{3}$ & KPHENO2NO2 & Jagiella and Zabel $(2007)^{*}$ \\
\hline Aro357 & TrGCN & $\mathrm{C} 6 \mathrm{H} 5 \mathrm{O} 2+\mathrm{NO}_{3} \rightarrow \mathrm{C} 6 \mathrm{H} 5 \mathrm{O}+\mathrm{NO}_{2}$ & KRO2NO3 & Rickard and Pascoe (2009) \\
\hline Aro358 & TrGC & $\mathrm{C} 6 \mathrm{H} 5 \mathrm{O} 2 \rightarrow \mathrm{C} 6 \mathrm{H} 5 \mathrm{O}$ & 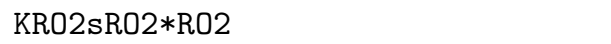 & Rickard and Pascoe $(\overline{2009})$ \\
\hline Aro359 & TrGC & $\mathrm{BZEMUCOH}+\mathrm{OH} \rightarrow \mathrm{BZEMUCCO}+\mathrm{HO}_{2}$ & $8.23 \mathrm{E}-11$ & Rickard and Pascoe (2009) \\
\hline Aro360 & TrGCN & $\mathrm{BZEMUCNO} 3+\mathrm{OH} \rightarrow \mathrm{BZEMUCCO}+\mathrm{NO}_{2}$ & $4.38 \mathrm{E}-11$ & Rickard and Pascoe 2009) \\
\hline Aro361 & $\operatorname{TrGC}$ & $\begin{array}{l}\text { TOLUENE }+\mathrm{OH} \rightarrow .07 \quad \mathrm{C} 6 \mathrm{H} 5 \mathrm{CH} 2 \mathrm{O} 2+.07 \\
\text { PTOL }_{\mathrm{C}} 6 \mathrm{H} 5 \mathrm{CH} 2 \mathrm{O} 2+.18 \mathrm{CRESOL}+.18 \mathrm{PTOL}_{\mathrm{C}} \text { RESOL } \\
+.18 \mathrm{HO}_{2}+.65 \text { TLBIPERO2 }+.65 \mathrm{PTOL}_{\mathrm{T}} \text { LBIPERO } 2 \\
+.10 \mathrm{TLEPOXMUC}^{+}+.10 \text { PTOL }_{\mathrm{T}} \text { LEPOXMUC }+.10 \\
\mathrm{HO}_{2}\end{array}$ & 1.8E-12*EXP (340/TEMP) & $\overline{\text { Rickard and Pascoe }}(\overline{\overline{2009}})^{*}$ \\
\hline Aro362 & $\operatorname{TrGC}$ & BZBIPERO2 $+\mathrm{HO}_{2} \rightarrow$ BZBIPEROOH & KRO2HO2*0.770 & Rickard and Pascoe 2009) \\
\hline Aro363 & TrGCN & BZBIPERO2 $+\mathrm{NO} \rightarrow$ BZBIPERNO3 & KR02NO*0.082 & $\overline{\text { Rickard and Pascoe }}(\overline{2009})$ \\
\hline Aro364 & TrGCN & $\begin{array}{l}\text { BZBIPERO2 }+\mathrm{NO} \rightarrow \mathrm{NO}_{2}+\text { GLYOX }+\mathrm{HO}_{2}+.5 \\
\text { BZFUONE }+.5 \text { BZFUONE }\end{array}$ & KR02NO*0.918 & $\overline{\text { Rickard and Pascoe }}(\overline{\overline{2009}})^{*}$ \\
\hline Aro365 & TrGCN & $\begin{array}{l}\text { BZBIPERO2 }+\mathrm{NO}_{3} \rightarrow \mathrm{NO}_{2}+\text { GLYOX }+\mathrm{HO}_{2}+.5 \\
\text { BZFUONE }+.5 \text { BZFUONE }\end{array}$ & KRO2NO3 & Rickard and Pascoe $(2009)^{*}$ \\
\hline Aro366 & TrGC & BZBIPERO $2 \rightarrow$ GLYOX $+\mathrm{HO}_{2}+$ BZFUONE & $8.80 \mathrm{E}-13 * \mathrm{R} 02$ & Rickard and Pascoe $(2009)^{*}$ \\
\hline Aro367 & TrGC & $\mathrm{C} 6 \mathrm{H} 5 \mathrm{CH} 2 \mathrm{O} 2+\mathrm{HO}_{2} \rightarrow \mathrm{C} 6 \mathrm{H} 5 \mathrm{CH} 2 \mathrm{OOH}$ & $1.5 \mathrm{E}-13 * \operatorname{EXP}(1310 / \mathrm{TEMP})$ & Rickard and Pascoe (2009) \\
\hline Aro368 & TrGCN & $\mathrm{C} 6 \mathrm{H} 5 \mathrm{CH} 2 \mathrm{O} 2+\mathrm{NO} \rightarrow \mathrm{C} 6 \mathrm{H} 5 \mathrm{CH} 2 \mathrm{NO} 3$ & KR02NO $* 0.105$ & Rickard and Pascoe $(2009)^{*}$ \\
\hline Aro369 & TrGCN & $\mathrm{C} 6 \mathrm{H} 5 \mathrm{CH} 2 \mathrm{O} 2+\mathrm{NO} \rightarrow$ BENZAL $+\mathrm{HO}_{2}+\mathrm{NO}_{2}$ & KRO2NO $* 0.985$ & Rickard and Pascoe $(\overline{2009})^{*}$ \\
\hline Aro370 & TrGCN & $\mathrm{C} 6 \mathrm{H} 5 \mathrm{CH} 2 \mathrm{O} 2+\mathrm{NO}_{3} \rightarrow \mathrm{BENZAL}+\mathrm{HO}_{2}+\mathrm{NO}_{2}$ & KRO2NO3 & $\overline{\text { Rickard and Pascoe }}(\overline{\overline{2009}})^{*}$ \\
\hline Aro371 & $\operatorname{TrGC}$ & $\mathrm{C} 6 \mathrm{H} 5 \mathrm{CH} 2 \mathrm{O} 2 \rightarrow \mathrm{BENZAL}+\mathrm{HO}_{2}$ & $\begin{array}{l}2 *(\mathrm{KCH} 302 * 2.4 \mathrm{E}-14 * \mathrm{EXP}(1620 / \mathrm{TEMP})) \\
* * 0.5 * \mathrm{RO} 2\end{array}$ & Rickard and Pascoe (2009)* \\
\hline Aro372 & TrGCN & $\begin{array}{l}\text { CRESOL }+\mathrm{NO}_{3} \rightarrow .103 \mathrm{CRESO} 2+.103 \mathrm{HNO}_{3}+.506 \\
\mathrm{NCRESO} 2+.391 \mathrm{TOL} 1 \mathrm{O}+.391 \mathrm{HNO}_{3}\end{array}$ & $1.4 \mathrm{E}-11$ & Rickard and Pascoe $(2009)^{*}$ \\
\hline Aro373 & TrGC & $\begin{array}{l}\text { CRESOL }+\mathrm{OH} \rightarrow .2 \text { CRESO } 2+.727 \text { MCATECHOL }+ \\
.727 \mathrm{HO}_{2}+.073 \text { TOL1O }\end{array}$ & $4.65 \mathrm{E}-11$ & Rickard and Pascoe (2009)* \\
\hline Aro374 & TrGC & TLBIPERO2 $+\mathrm{HO}_{2} \rightarrow$ TLBIPEROOH & $\mathrm{KRO} 2 \mathrm{HO} 2 * 0.820$ & Rickard and Pascoe (2009) \\
\hline Aro375 & TrGCN & $\begin{array}{l}\text { TLBIPERO } 2+\mathrm{NO} \rightarrow \mathrm{NO}_{2}+.6 \text { GLYOX }+.4 \text { MGLYOX } \\
+\mathrm{HO}_{2}+.2 \mathrm{C} 4 \mathrm{MDIAL}+.2 \mathrm{C} 5 \mathrm{DICARB}+.2 \text { TLFUONE } \\
+.2 \text { BZFUONE }+.2 \mathrm{MALDIAL}\end{array}$ & KRO2NO $* 0.889$ & Rickard and Pascoe $(\overline{2009})^{*}$ \\
\hline Aro376 & TrGCN & TLBIPERO2 + NO $\rightarrow$ TLBIPERNO3 & $\mathrm{KRO} 2 \mathrm{NO} * 0.111$ & Rickard and Pascoe (2009) \\
\hline
\end{tabular}


Table 1: Gas phase reactions (... continued)

\begin{tabular}{|c|c|c|c|c|}
\hline \# & labels & chemical reaction & rate coefficient & reference \\
\hline Aro377 & TrGCN & $\begin{array}{l}\text { TLBIPERO } 2+\mathrm{NO}_{3} \rightarrow \mathrm{NO}_{2}+.6 \mathrm{GLYOX}+.4 \text { MGLYOX } \\
+\mathrm{HO}_{2}+.2 \mathrm{C} 4 \mathrm{MDIAL}+.2 \mathrm{C} 5 \mathrm{DICARB}+.2 \text { TLFUONE } \\
+.2 \mathrm{BZFUONE}+.2 \mathrm{MALDIAL}\end{array}$ & KR02NO3 & Rickard and Pascoe $(2009)^{*}$ \\
\hline Aro378 & TrGC & $\begin{array}{l}\text { TLBIPERO } 2 \rightarrow .6 \text { GLYOX }+.4 \text { MGLYOX }+\mathrm{HO}_{2}+ \\
.2 \text { C4MDIAL }+.2 \text { C5DICARB }+.2 \text { TLFUONE }+.2 \\
\text { BZFUONE }+.2 \text { MALDIAL }\end{array}$ & $8.80 \mathrm{E}-13 * \mathrm{R} 02$ & Rickard and Pascoe $(2009)^{*}$ \\
\hline Aro379 & TrGCN & TLEPOXMUC $+\mathrm{NO}_{3} \rightarrow$ TLEMUCCO $3+\mathrm{HNO}_{3}$ & KNO3AL $* 2.75$ & Rickard and Pascoe (2009) \\
\hline Aro380 & $\operatorname{TrGC}$ & $\begin{array}{l}\text { TLEPOXMUC }+\mathrm{O}_{3} \rightarrow \text { EPXC4DIAL }+.125 \mathrm{CH}_{3} \mathrm{CHO}+ \\
.695 \mathrm{CH}_{3} \mathrm{C}(\mathrm{O}) \mathrm{OO}+.57 \mathrm{CO}+.57 \mathrm{OH}+.125 \mathrm{HO}_{2}+.1125 \\
\mathrm{CH} 3 \mathrm{COCO} 2 \mathrm{H}+.0675 \mathrm{MGLYOX}+.0675 \mathrm{H}_{2} \mathrm{O}_{2}\end{array}$ & $5.00 \mathrm{E}-18$ & Rickard and Pascoe $(2009) *$ \\
\hline Aro381 & $\operatorname{TrGC}$ & $\begin{array}{l}\text { TLEPOXMUC }+\mathrm{OH} \rightarrow .31 \text { TLEMUCCO } 3+.69 \\
\text { TLEMUCO } 2\end{array}$ & $7.99 \mathrm{E}-11$ & Rickard and Pascoe $(2009)^{*}$ \\
\hline Aro382 & $\operatorname{TrGC}$ & $\mathrm{C} 6 \mathrm{H} 5 \mathrm{CH} 2 \mathrm{OOH}+\mathrm{OH} \rightarrow \mathrm{BENZAL}+\mathrm{OH}$ & $2.05 \mathrm{E}-11$ & Rickard and Pascoe (2009) \\
\hline Aro383 & TrGCN & $\mathrm{C} 6 \mathrm{H} 5 \mathrm{CH} 2 \mathrm{NO} 3+\mathrm{OH} \rightarrow \mathrm{BENZAL}+\mathrm{NO}_{2}$ & $6.03 \mathrm{E}-12$ & Rickard and Pascoe $(\overline{\overline{2009}})$ \\
\hline Aro384 & TrGCN & $\mathrm{BENZAL}+\mathrm{NO}_{3} \rightarrow \mathrm{C} 6 \mathrm{H} 5 \mathrm{CO} 3+\mathrm{HNO}_{3}$ & $2.40 \mathrm{E}-15$ & $\overline{\text { Rickard and Pascoe }}(\overline{\overline{2009}})$ \\
\hline Aro385 & TrGC & $\mathrm{BENZAL}+\mathrm{OH} \rightarrow \mathrm{C} 6 \mathrm{H} 5 \mathrm{CO} 3$ & $5.9 \mathrm{E}-12 * \operatorname{EXP}(225 / \mathrm{TEMP})$ & Rickard and Pascoe $(\overline{\overline{2009}})$ \\
\hline Aro386 & TrGC & $\mathrm{CRESO} 2+\mathrm{HO}_{2} \rightarrow \mathrm{CRESOOH}$ & KRO2HO2*0.820*(1-rchohch2o2_oh) & Rickard and Pascoe $(\overline{2009})$ \\
\hline Aro387 & $\operatorname{TrGC}$ & $\begin{array}{l}\mathrm{CRESO} 2+\mathrm{HO}_{2} \rightarrow .68 \mathrm{C} 5 \mathrm{CO} 14 \mathrm{OH}+.68 \mathrm{GLYOX}+\mathrm{HO}_{2} \\
+.32 \text { PTLQONE }+\mathrm{OH}\end{array}$ & KRO2HO2*0.820*rchohch2o2_oh & Rickard and Pascoe $(\overline{2009})^{*}$ \\
\hline Aro388 & TrGCN & $\begin{array}{l}\mathrm{CRESO} 2+\mathrm{NO} \rightarrow .68 \mathrm{C} 5 \mathrm{CO} 14 \mathrm{OH}+.68 \mathrm{GLYOX}+\mathrm{HO}_{2} \\
+.32 \mathrm{PTLQONE}+\mathrm{NO}_{2}\end{array}$ & KRO2NO & Rickard and Pascoe $(2009)^{*}$ \\
\hline Aro389 & TrGCN & $\begin{array}{l}\mathrm{CRESO} 2+\mathrm{NO}_{3} \rightarrow .68 \mathrm{C} 5 \mathrm{CO} 14 \mathrm{OH}+.68 \mathrm{GLYOX}+\mathrm{HO}_{2} \\
+.32 \mathrm{PTLQONE}+\mathrm{NO}_{2}\end{array}$ & KR02NO3 & Rickard and Pascoe $(2009)^{*}$ \\
\hline Aro390 & TrGC & $\begin{array}{l}\mathrm{CRESO} 2 \rightarrow .68 \mathrm{C} 5 \mathrm{CO} 14 \mathrm{OH}+.68 \mathrm{GLYOX}+\mathrm{HO}_{2}+.32 \\
\text { PTLQONE }\end{array}$ & $8.00 \mathrm{E}-13 * \mathrm{R} 02$ & Rickard and Pascoe $(2009)^{*}$ \\
\hline Aro391 & TrGCN & $\mathrm{NCRESO} 2+\mathrm{HO}_{2} \rightarrow \mathrm{NCRESOOH}$ & KRO2HO2 $* 0.820 *\left(1-r c h o h c h 202 \_o h\right)$ & Rickard and Pascoe (2009) \\
\hline Aro392 & TrGCN & $\begin{array}{l}\mathrm{NCRESO} 2+\mathrm{HO}_{2} \rightarrow \mathrm{C} 5 \mathrm{CO} 14 \mathrm{OH}+\mathrm{GLYOX}+\mathrm{NO}_{2}+ \\
\mathrm{OH}\end{array}$ & KRO2HO2*0.820*rchohch2o2_oh & $\overline{\text { Rickard and Pascoe }}(\overline{\overline{2009}})^{*}$ \\
\hline Aro393 & TrGCN & $\mathrm{NCRESO} 2+\mathrm{NO} \rightarrow \mathrm{C} 5 \mathrm{CO} 14 \mathrm{OH}+\mathrm{GLYOX}+\mathrm{NO}_{2}+\mathrm{NO}_{2}$ & KRO2NO & Rickard and Pascoe $(2009)^{*}$ \\
\hline Aro394 & TrGCN & $\begin{array}{l}\mathrm{NCRESO} 2+\mathrm{NO}_{3} \rightarrow \mathrm{C} 5 \mathrm{CO} 14 \mathrm{OH}+\mathrm{GLYOX}+\mathrm{NO}_{2}+ \\
\mathrm{NO}_{2}\end{array}$ & KRO2NO3 & Rickard and Pascoe $(\overline{2009})^{*}$ \\
\hline Aro395 & TrGCN & $\mathrm{NCRESO} 2 \rightarrow \mathrm{C} 5 \mathrm{CO} 14 \mathrm{OH}+\mathrm{GLYOX}+\mathrm{NO}_{2}$ & $8.00 \mathrm{E}-13 * \mathrm{R} 02$ & Rickard and Pascoe 2009)* \\
\hline Aro396 & TrGCN & $\mathrm{TOL} 1 \mathrm{O}+\mathrm{NO}_{2} \rightarrow \mathrm{TOL} 1 \mathrm{OHNO} 2$ & $2.08 \mathrm{E}-12$ & $\overline{\text { Rickard and Pascoe }}(\overline{\overline{2009}})^{*}$ \\
\hline Aro397 & $\operatorname{TrGC}$ & $\mathrm{TOL} 1 \mathrm{O}+\mathrm{O}_{3} \rightarrow \mathrm{OXYL} 1 \mathrm{O} 2$ & $2.86 \mathrm{E}-13$ & Rickard and Pascoe (2009) \\
\hline Aro398 & TrGCN & $\mathrm{MCATECHOL}+\mathrm{NO}_{3} \rightarrow \mathrm{MCATEC} 1 \mathrm{O}+\mathrm{HNO}_{3}$ & $1.7 \mathrm{E}-10 * 1.0$ & Rickard and Pascoe $(\overline{2009})$ \\
\hline
\end{tabular}


Table 1: Gas phase reactions (... continued)

\begin{tabular}{|c|c|c|c|c|}
\hline$\#$ & labels & chemical reaction & rate coefficient & reference \\
\hline Aro399 & TrGC & $\begin{array}{l}\mathrm{MCATECHOL}+\mathrm{O}_{3} \rightarrow \mathrm{MC} 3 \mathrm{ODBCO} 2 \mathrm{H}+\mathrm{HCOCO}_{2} \mathrm{H}+ \\
\mathrm{HO}_{2}+\mathrm{OH}\end{array}$ & $2.8 \mathrm{E}-17$ & Rickard and Pascoe $(2009)^{*}$ \\
\hline Aro400 & TrGC & MCATECHOL + OH $\rightarrow$ MCATEC1O & $2.0 \mathrm{E}-10 * 1.0$ & Rickard and Pascoe (2009) \\
\hline Aro401 & TrGC & TLBIPEROOH $+\mathrm{OH} \rightarrow$ TLOBIPEROH + OH & $9.64 \mathrm{E}-11$ & Rickard and Pascoe (2009) \\
\hline Aro402 & TrGCN & TLBIPERNO3 $+\mathrm{OH} \rightarrow$ TLOBIPEROH $+\mathrm{NO}_{2}$ & $7.16 \mathrm{E}-11$ & Rickard and Pascoe $(\overline{2009})$ \\
\hline Aro403 & $\operatorname{TrGC}$ & TLOBIPEROH + OH $\rightarrow$ C5CO14O2 + GLYOX & $7.99 \mathrm{E}-11$ & Rickard and Pascoe (2009) \\
\hline Aro404 & TrGC & TLEMUCCO $3+\mathrm{HO}_{2} \rightarrow \mathrm{C} 615 \mathrm{CO} 2 \mathrm{O} 2+\mathrm{OH}$ & KAPHO $2 *$ rco3_oh & Rickard and Pascoe $(\overline{2009})^{*}$ \\
\hline Aro405 & TrGC & TLEMUCCO $3+\mathrm{HO}_{2} \rightarrow$ TLEMUCCO $2 \mathrm{H}+\mathrm{O}_{3}$ & KAPHO $2 * r c 03 \_$o3 & $\overline{\text { Rickard and Pascoe }}(\overline{\overline{2009}})^{*}$ \\
\hline Aro406 & TrGCN & TLEMUCCO3 $+\mathrm{HO}_{2} \rightarrow$ TLEMUCCO3H & KAPHO2*rco3_ooh & Rickard and Pascoe $(\overline{2009})^{*}$ \\
\hline Aro407 & TrGCN & TLEMUCCO $3+\mathrm{NO} \rightarrow \mathrm{C} 615 \mathrm{CO} 2 \mathrm{O} 2+\mathrm{NO}_{2}$ & KAPNO & Rickard and Pascoe \\
\hline Aro408 & TrGCN & TLEMUCCO3 $+\mathrm{NO}_{2} \rightarrow$ TLEMUCPAN & k_CH3CO3_NO2 & Rickard and Pascoe $(\overline{2009})^{*}$ \\
\hline Aro409 & TrGCN & TLEMUCCO $3+\mathrm{NO}_{3} \rightarrow \mathrm{C} 615 \mathrm{CO} 2 \mathrm{O} 2+\mathrm{NO}_{2}$ & KRO2NO3*1.74 & $\overline{\text { Rickard and Pascoe }}(\overline{\overline{2009}})$ \\
\hline Aro410 & $\operatorname{TrGC}$ & TLEMUCCO3 $\rightarrow$ C615CO2O2 & KRO2AP*R02 & Rickard and Pascoe (2009)* \\
\hline Aro411 & $\operatorname{TrGC}$ & TLEMUCO $2+\mathrm{HO}_{2} \rightarrow$ TLEMUCOOH & $\begin{array}{l}\mathrm{KRO} 2 \mathrm{HO} 2 * 0.820 *\left(1-\mathrm{rchohch} 2 \mathrm{o} 2_{-}\right. \\
\text {oh-rcoch2o2_oh) }\end{array}$ & Rickard and Pascoe $(\overline{2009})$ \\
\hline Aro412 & $\operatorname{TrGC}$ & $\begin{array}{l}\text { TLEMUCO } 2+\mathrm{HO}_{2} \rightarrow .5 \mathrm{C} 3 \mathrm{DIALO} 2+.5 \mathrm{CO} 2 \mathrm{H} 3 \mathrm{CHO}+ \\
.5 \text { EPXC4DIAL }+.5 \mathrm{MGLYOX}+.5 \mathrm{HO}_{2}+\mathrm{OH}\end{array}$ & $\begin{array}{l}\mathrm{KRO} 2 \mathrm{HO} 2 * 0.820 *\left(\mathrm{rchohch} 2 \mathrm{o} 2_{-} \mathrm{oh}+\mathrm{rcoch} 2 \mathrm{o} 2_{-}\right. \\
\text {oh) }\end{array}$ & Rickard and Pascoe (2009)* \\
\hline Aro413 & TrGCN & TLEMUCO2 + NO $\rightarrow$ TLEMUCNO3 & KRO2NO*0.105 & Rickard and Pascoe 2009) \\
\hline Aro414 & TrGCN & $\begin{array}{l}\text { TLEMUCO } 2+\mathrm{NO} \rightarrow .5 \mathrm{C} 3 \mathrm{DIALO} 2+.5 \mathrm{CO} 2 \mathrm{H} 3 \mathrm{CHO}+ \\
.5 \mathrm{EPXC} 4 \mathrm{DIAL}+.5 \mathrm{MGLYOX}+.5 \mathrm{HO}_{2}+\mathrm{NO}_{2}\end{array}$ & KR02NO*0.985 & Rickard and Pascoe $(\overline{2009})^{*}$ \\
\hline Aro415 & TrGCN & $\begin{array}{l}\text { TLEMUCO } 2+\mathrm{NO}_{3} \rightarrow .5 \mathrm{C} 3 \mathrm{DIALO} 2+.5 \mathrm{CO} 2 \mathrm{H} 3 \mathrm{CHO}+ \\
.5 \text { EPXC4DIAL }+.5 \text { MGLYOX }+.5 \mathrm{HO}_{2}+\mathrm{NO}_{2}\end{array}$ & KR02NO3 & Rickard and Pascoe (2009)* \\
\hline Aro416 & TrGCN & $\begin{array}{l}\text { TLEMUCO } 2 \rightarrow .5 \text { C3DIALO2 }+.5 \mathrm{CO} 2 \mathrm{H} 3 \mathrm{CHO}+.5 \\
\text { EPXC4DIAL }+.5 \mathrm{MGLYOX}+.5 \mathrm{HO}_{2}\end{array}$ & $8.80 \mathrm{E}-13 * \mathrm{R} 02$ & Rickard and Pascoe $(2009)^{*}$ \\
\hline Aro417 & $\operatorname{TrGC}$ & $\mathrm{C} 6 \mathrm{H} 5 \mathrm{CO} 3+\mathrm{HO}_{2} \rightarrow \mathrm{C} 6 \mathrm{H} 5 \mathrm{CO} 3 \mathrm{H}$ & KAPHO2 $2 * 0.065$ & Roth et al. $(2010)^{*}$ \\
\hline Aro418 & $\operatorname{TrGC}$ & $\mathrm{C} 6 \mathrm{H} 5 \mathrm{CO} 3+\mathrm{HO}_{2} \rightarrow \mathrm{C} 6 \mathrm{H} 5 \mathrm{O} 2+\mathrm{OH}$ & KAPHO $2 * 0.20$ & Roth et al. $(\overline{2010})^{*}$ \\
\hline Aro419 & TrGC & $\mathrm{C} 6 \mathrm{H} 5 \mathrm{CO} 3+\mathrm{HO}_{2} \rightarrow \mathrm{PHCOOH}+\mathrm{O}_{3}$ & KAPHO $2 * 0.15$ & Roth et al. $(\overline{2010})^{*}$ \\
\hline Aro420 & TrGCN & $\mathrm{C} 6 \mathrm{H} 5 \mathrm{CO} 3+\mathrm{NO} \rightarrow \mathrm{C} 6 \mathrm{H} 5 \mathrm{O} 2+\mathrm{NO}_{2}$ & KAPNO & Rickard and Pascoe 2009) \\
\hline Aro421 & TrGCN & $\mathrm{C} 6 \mathrm{H} 5 \mathrm{CO} 3+\mathrm{NO}_{2} \rightarrow \mathrm{PBZN}$ & k_CH3CO3_NO2 & Rickard and Pascoe (2009)* \\
\hline Aro422 & TrGCN & $\mathrm{C} 6 \mathrm{H} 5 \mathrm{CO} 3+\mathrm{NO}_{3} \rightarrow \mathrm{C} 6 \mathrm{H} 5 \mathrm{O} 2+\mathrm{NO}_{2}$ & KRO2NO3*1.74 & Rickard and Pascoe 2009 \\
\hline Aro423 & TrGC & $\mathrm{C} 6 \mathrm{H} 5 \mathrm{CO} 3 \rightarrow \mathrm{C} 6 \mathrm{H} 5 \mathrm{O} 2$ & KR02AP*R02 & Rickard and Pascoe (2009) \\
\hline Aro424 & $\operatorname{TrGC}$ & $\mathrm{CRESOOH}+\mathrm{OH} \rightarrow \mathrm{CRESO} 2$ & $1.15 \mathrm{E}-10$ & Rickard and Pascoe (2009) \\
\hline Aro425 & TrGCN & $\mathrm{NCRESOOH}+\mathrm{OH} \rightarrow \mathrm{NCRESO} 2$ & $1.07 \mathrm{E}-10$ & Rickard and Pascoe $(\overline{2009})$ \\
\hline Aro426 & TrGCN & TOL1OHNO2 $+\mathrm{NO}_{3} \rightarrow \mathrm{NCRES1O}+\mathrm{HNO}_{3}$ & $3.13 E-13 * 1.0$ & Rickard and Pascoe \\
\hline Aro427 & TrGCN & TOL1OHNO2 + OH $\rightarrow$ NCRES1O & $2.8 \mathrm{E}-12$ & Rickard and Pascoe (2009) \\
\hline
\end{tabular}


Table 1: Gas phase reactions (... continued)

\begin{tabular}{|c|c|c|c|c|}
\hline \# & labels & chemical reaction & rate coefficient & reference \\
\hline Aro428 & TrGC & $\mathrm{OXYL} 1 \mathrm{O} 2+\mathrm{HO}_{2} \rightarrow \mathrm{OXYL1OOH}$ & $\mathrm{KRO} 2 \mathrm{HO} 2 * 0.820$ & Rickard and Pascoe $(2009)$ \\
\hline Aro429 & TrGCN & $\mathrm{OXYL} 1 \mathrm{O} 2+\mathrm{NO} \rightarrow \mathrm{TOL} 1 \mathrm{O}+\mathrm{NO}_{2}$ & KRO2NO & $\overline{\text { Rickard and Pascoe }}(\overline{\overline{2009}})$ \\
\hline Aro430 & TrGCN & $\mathrm{OXYL} 1 \mathrm{O} 2+\mathrm{NO}_{2} \rightarrow \mathrm{TOL} 1 \mathrm{O}+\mathrm{NO}_{3}$ & KPHENO2NO2 & Jagiella and Zabel $(2007)^{*}$ \\
\hline Aro431 & TrGCN & $\mathrm{OXYL} 1 \mathrm{O} 2+\mathrm{NO}_{3} \rightarrow \mathrm{TOL} 1 \mathrm{O}+\mathrm{NO}_{2}$ & KRO2NO3 & Rickard and Pascoe $(2009)$ \\
\hline Aro432 & TrGC & OXYL1O2 $\rightarrow$ TOL1O & KR02sR02*R02 & $\overline{\text { Rickard and Pascoe }}(\overline{\overline{2009}})$ \\
\hline Aro433 & TrGCN & $\mathrm{MCATEC} 1 \mathrm{O}+\mathrm{NO}_{2} \rightarrow$ MNCATECH & $2.08 \mathrm{E}-12$ & Rickard and Pascoe $(\overline{2009})$ \\
\hline Aro434 & $\operatorname{TrGC}$ & $\mathrm{MCATEC} 1 \mathrm{O}+\mathrm{O}_{3} \rightarrow \mathrm{MCATEC} 1 \mathrm{O} 2$ & $2.86 \mathrm{E}-13$ & $\overline{\text { Rickard and Pascoe }}(\overline{\overline{2009}})$ \\
\hline Aro435 & TrGCAro & $\mathrm{C} 4 \mathrm{MDIAL}+\mathrm{NO}_{3} \rightarrow \mathrm{MC} 3 \mathrm{CODBCO} 3+\mathrm{HNO}_{3}$ & $\mathrm{KNO3AL} * 4.25$ & $\overline{\text { Rickard and Pascoe }}(\overline{\overline{2009}})$ \\
\hline Aro436 & TrGCAro & $\mathrm{C} 4 \mathrm{MDIAL}+\mathrm{NO}_{3} \rightarrow \mathrm{C} 3 \mathrm{MCODBCO} 3+\mathrm{HNO}_{3}$ & $\mathrm{KNO} 3 \mathrm{AL} * 4.25$ & $\overline{\text { Rickard and Pascoe }}(\overline{2009})$ \\
\hline Aro437 & TrGCN & $\begin{array}{l}\text { C4MDIAL }+\mathrm{O}_{3} \rightarrow .445 \mathrm{OH}+.445 \mathrm{CO}+.445 \\
\mathrm{CH}_{3} \mathrm{C}(\mathrm{O}) \mathrm{OO}+.055 \mathrm{MGLYOX}+.055 \mathrm{H}_{2} \mathrm{O}_{2}+.5 \mathrm{GLYOX} \\
+.5 \mathrm{MGLYOX}+.0343 \mathrm{HCOCO} \mathrm{H}_{2}+.0206 \mathrm{GLYOX}+ \\
.0206 \mathrm{H}_{2} \mathrm{O}_{2}+.445 \mathrm{OH}+.445 \mathrm{HO}_{2}+.445 \mathrm{CO}+.445 \mathrm{CO}\end{array}$ & $5.00 \mathrm{E}-18$ & $\overline{\text { Rickard and Pascoe }}(\overline{\overline{2009}})$ \\
\hline Aro438 & $\operatorname{TrGC}$ & $\begin{array}{l}\text { C4MDIAL }+\mathrm{OH} \rightarrow .385 \text { C3MCODBCO3 }+.23 \\
\text { C4M2ALOHO2 }+.385 \text { MC3CODBCO3 }\end{array}$ & $4.41 \mathrm{E}-11$ & Rickard and Pascoe (2009) \\
\hline Aro439 & TrGC & TLEMUCCO2H $+\mathrm{OH} \rightarrow \mathrm{C} 615 \mathrm{CO} 2 \mathrm{O} 2$ & $5.98 \mathrm{E}-11$ & Rickard and Pascoe (2009) \\
\hline Aro440 & TrGC & TLEMUCCO3H + OH $\rightarrow$ TLEMUCCO3 & $6.29 \mathrm{E}-11$ & Rickard and Pascoe $(\overline{2009})$ \\
\hline Aro441 & TrGCN & TLEMUCPAN $+\mathrm{OH} \rightarrow \mathrm{C} 5 \mathrm{DICARB}+\mathrm{CO}+\mathrm{NO}_{2}$ & $5.96 \mathrm{E}-11$ & Rickard and Pascoe (2009) \\
\hline Aro442 & $\operatorname{TrGCN}$ & TLEMUCPAN $\rightarrow$ TLEMUCCO $3+\mathrm{NO}_{2}$ & k_PAN_M & $\overline{\text { Rickard and Pascoe }}(\overline{\overline{2009}})^{*}$ \\
\hline Aro443 & TrGC & TLEMUCOOH $+\mathrm{OH} \rightarrow$ TLEMUCCO $+\mathrm{OH}$ & $7.04 \mathrm{E}-11$ & Rickard and Pascoe \\
\hline Aro444 & TrGCN & TLEMUCNO3 $+\mathrm{OH} \rightarrow$ TLEMUCCO $+\mathrm{NO}_{2}$ & $3.06 \mathrm{E}-11$ & Rickard and Pascoe \\
\hline Aro445 & TrGC & $\begin{array}{l}\text { TLEMUCCO }+\mathrm{OH} \rightarrow \mathrm{CH}_{3} \mathrm{C}(\mathrm{O}) \mathrm{OO}+\mathrm{EPXC} 4 \mathrm{DIAL}+ \\
\mathrm{CO}\end{array}$ & $4.06 \mathrm{E}-11$ & Rickard and Pascoe $(\overline{2009})$ \\
\hline Aro446 & TrGC & $\mathrm{C} 6 \mathrm{H} 5 \mathrm{CO} 3 \mathrm{H}+\mathrm{OH} \rightarrow \mathrm{C} 6 \mathrm{H} 5 \mathrm{CO} 3$ & $4.66 \mathrm{E}-12$ & Rickard and Pascoe (2009) \\
\hline Aro447 & TrGC & $\mathrm{PHCOOH}+\mathrm{OH} \rightarrow \mathrm{C} 6 \mathrm{H} 5 \mathrm{O} 2$ & $1.10 \mathrm{E}-12$ & $\overline{\text { Rickard and Pascoe }}(\overline{\overline{2009}})$ \\
\hline Aro448 & TrGCN & $\mathrm{PBZN}+\mathrm{OH} \rightarrow \mathrm{C} 6 \mathrm{H} 5 \mathrm{OOH}+\mathrm{CO}+\mathrm{NO}_{2}$ & $1.06 \mathrm{E}-12$ & Rickard and Pascoe $(\overline{2009})$ \\
\hline Aro449 & TrGCN & $\mathrm{PBZN} \rightarrow \mathrm{C} 6 \mathrm{H} 5 \mathrm{CO} 3+\mathrm{NO}_{2}$ & k_PAN_M*0.67 & $\overline{\text { Rickard and Pascoe }}(\overline{\overline{2009}})^{*}$ \\
\hline Aro450 & TrGCN & $\mathrm{PTLQONE}+\mathrm{NO}_{3} \rightarrow \mathrm{NPTLQO} 2$ & $1.00 \mathrm{E}-12$ & Rickard and Pascoe (2009) \\
\hline Aro451 & TrGC & PTLQONE + OH $\rightarrow$ PTLQO2 & $2.3 \mathrm{E}-11$ & $\overline{\text { Rickard and Pascoe }}(\overline{\overline{2009}})$ \\
\hline Aro452 & TrGCN & $\mathrm{NCRES} 1 \mathrm{O}+\mathrm{NO}_{2} \rightarrow$ DNCRES & $2.08 \mathrm{E}-12$ & Rickard and Pascoe $(\overline{2009})$ \\
\hline Aro453 & TrGCN & $\mathrm{NCRES} 1 \mathrm{O}+\mathrm{O}_{3} \rightarrow \mathrm{NCRES} 1 \mathrm{O} 2$ & $2.86 \mathrm{E}-13$ & Rickard and Pascoe $(\overline{2009})$ \\
\hline Aro454 & TrGC & OXYL1OOH + OH $\rightarrow$ OXYL1O2 & $4.65 \mathrm{E}-11$ & Rickard and Pascoe $(\overline{2009})$ \\
\hline Aro455 & TrGCN & MNCATECH $+\mathrm{NO}_{3} \rightarrow$ MNNCATECO2 & $5.03 \mathrm{E}-12$ & Rickard and Pascoe $(\overline{2009})$ \\
\hline Aro456 & TrGCN & MNCATECH + OH $\rightarrow$ MNCATECO2 & $6.83 \mathrm{E}-12$ & Rickard and Pascoe $(\overline{2009})$ \\
\hline Aro457 & TrGC & $\mathrm{MCATEC} 1 \mathrm{O} 2+\mathrm{HO}_{2} \rightarrow$ MCATEC1OOH & KRO2HO2*0.820*(1-rchohch2o2_oh) & Rickard and Pascoe $(\overline{2009})$ \\
\hline
\end{tabular}


Table 1: Gas phase reactions (... continued)

\begin{tabular}{|c|c|c|c|c|}
\hline \# & labels & chemical reaction & rate coefficient & reference \\
\hline Aro458 & TrGC & $\mathrm{MCATEC} 1 \mathrm{O} 2+\mathrm{HO}_{2} \rightarrow \mathrm{MCATEC} 1 \mathrm{O}+\mathrm{OH}$ & KR02H02*0.820*rchohch2o2_oh & Rickard and Pascoe 2009$)^{*}$ \\
\hline Aro459 & TrGCN & $\mathrm{MCATEC} 1 \mathrm{O} 2+\mathrm{NO} \rightarrow \mathrm{MCATEC} 1 \mathrm{O}+\mathrm{NO}_{2}$ & KRO2NO & $\overline{\text { Rickard and Pascoe }}(\overline{\overline{2009}})$ \\
\hline Aro460 & TrGCN & $\mathrm{MCATEC} 1 \mathrm{O} 2+\mathrm{NO}_{2} \rightarrow \mathrm{MCATEC} 1 \mathrm{O}+\mathrm{NO}_{3}$ & KPHENO2NO2 & Jagiella and Zabel (2007)* \\
\hline Aro461 & TrGCN & $\mathrm{MCATEC} 1 \mathrm{O} 2+\mathrm{NO}_{3} \rightarrow \mathrm{MCATEC} 1 \mathrm{O}+\mathrm{NO}_{2}$ & KR02NO3 & Rickard and Pascoe (2009) \\
\hline Aro462 & TrGC & MCATEC1O2 $\rightarrow$ MCATEC1O & $8.80 \mathrm{E}-13 * \mathrm{R} 02$ & $\overline{\text { Rickard and Pascoe }}(\overline{\overline{2009}})$ \\
\hline Aro463 & TrGCN & $\mathrm{NPTLQO} 2+\mathrm{HO}_{2} \rightarrow$ NPTLQOOH & KRO2HO2*0.820*(1-rcoch2o2_oh $)$ & Rickard and Pascoe $(\overline{2009})$ \\
\hline Aro464 & TrGCN & $\mathrm{NPTLQO} 2+\mathrm{HO}_{2} \rightarrow \mathrm{C} 7 \mathrm{CO} 4 \mathrm{DB}+\mathrm{NO}_{2}+\mathrm{OH}$ & KRO2HO2*0.820*rcoch 202 _oh & Rickard and Pascoe $(\overline{2009})^{*}$ \\
\hline Aro465 & $\operatorname{TrGCN}$ & $\mathrm{NPTLQO} 2+\mathrm{NO} \rightarrow \mathrm{C} 7 \mathrm{CO} 4 \mathrm{DB}+\mathrm{NO}_{2}+\mathrm{NO}_{2}$ & KRO2NO & $\overline{\text { Rickard and Pascoe }}(\overline{\overline{2009}})^{*}$ \\
\hline Aro466 & TrGCN & $\mathrm{NPTLQO} 2+\mathrm{NO}_{3} \rightarrow \mathrm{C} 7 \mathrm{CO} 4 \mathrm{DB}+\mathrm{NO}_{2}+\mathrm{NO}_{2}$ & KRO2NO3 & $\overline{\text { Rickard and Pascoe }}(\overline{2009})^{*}$ \\
\hline Aro467 & TrGCN & $\mathrm{NPTLQO} 2 \rightarrow \mathrm{C} 7 \mathrm{CO} 4 \mathrm{DB}+\mathrm{NO}_{2}$ & $8.80 \mathrm{E}-13 * \mathrm{R} 02$ & Rickard and Pascoe $(\overline{2009})^{*}$ \\
\hline Aro468 & $\operatorname{TrGC}$ & $\mathrm{PTLQO} 2+\mathrm{HO}_{2} \rightarrow \mathrm{PTLQOOH}$ & $\begin{array}{l}\text { KRO2HO2*0.820* }\left(1-r c h o h c h 2 \circ 2_{-}\right. \\
\text {oh-rcoch2o2_oh) }\end{array}$ & Rickard and Pascoe $(\overline{2009})$ \\
\hline Aro469 & $\operatorname{TrGC}$ & $\mathrm{PTLQO} 2+\mathrm{HO}_{2} \rightarrow \mathrm{C} 6 \mathrm{CO} 2 \mathrm{OHCO} 3+\mathrm{OH}$ & $\begin{array}{l}\text { KRO2HO2*0.820*(rchohch2o2_oh }+ \text { rcoch } 202 \\
\text { oh) }\end{array}$ & Rickard and Pascoe (2009)* \\
\hline Aro470 & TrGCN & $\mathrm{PTLQO} 2+\mathrm{NO} \rightarrow \mathrm{C} 6 \mathrm{CO} 2 \mathrm{OHCO} 3+\mathrm{NO}_{2}$ & KRO2NO & Rickard and Pascoe (2009)* \\
\hline Aro471 & TrGCN & $\mathrm{PTLQO} 2+\mathrm{NO}_{3} \rightarrow \mathrm{C} 6 \mathrm{CO} 2 \mathrm{OHCO} 3+\mathrm{NO}_{2}$ & KR02NO3 & Rickard and Pascoe (2009)* \\
\hline Aro472 & TrGC & $\mathrm{PTLQO} 2 \rightarrow \mathrm{C} 6 \mathrm{CO} 2 \mathrm{OHCO} 3$ & $8.80 \mathrm{E}-13 * \mathrm{R} 02$ & Rickard and Pascoe $(\overline{2009})^{*}$ \\
\hline Aro473 & TrGCN & DNCRES $+\mathrm{NO}_{3} \rightarrow$ NDNCRESO 2 & $7.83 \mathrm{E}-15$ & Rickard and Pascoe (2009) \\
\hline Aro474 & TrGCN & DNCRES $+\mathrm{OH} \rightarrow$ DNCRESO2 & $5.10 \mathrm{E}-14$ & Rickard and Pascoe (2009) \\
\hline Aro475 & TrGCN & $\mathrm{NCRES} 1 \mathrm{O} 2+\mathrm{HO}_{2} \rightarrow \mathrm{NCRES} 1 \mathrm{OOH}$ & $\mathrm{KRO} 2 \mathrm{HO} 2 * 0.820$ & Rickard and Pascoe (2009) \\
\hline Aro476 & TrGCN & $\mathrm{NCRES} 1 \mathrm{O} 2+\mathrm{NO} \rightarrow \mathrm{NCRES} 1 \mathrm{O}+\mathrm{NO}_{2}$ & KRO2NO & Rickard and Pascoe $(\overline{2009})$ \\
\hline Aro477 & TrGCN & $\mathrm{NCRES} 1 \mathrm{O} 2+\mathrm{NO}_{2} \rightarrow \mathrm{NCRES} 1 \mathrm{O}+\mathrm{NO}_{3}$ & KPHENO2NO2 & Jagiella and Zabel (2007)* \\
\hline Aro478 & $\operatorname{TrGCN}$ & $\mathrm{NCRES} 1 \mathrm{O} 2+\mathrm{NO}_{3} \rightarrow \mathrm{NCRES} 1 \mathrm{O}+\mathrm{NO}_{2}$ & KRO2NO3 & Rickard and Pascoe $(2009)$ \\
\hline Aro479 & TrGCN & NCRES1O2 $\rightarrow$ NCRES1O & $\mathrm{KRO2sR02*R02}$ & Rickard and Pascoe $(\overline{2009})$ \\
\hline Aro480 & TrGCN & $\mathrm{MNNCATECO} 2+\mathrm{HO}_{2} \rightarrow \mathrm{MNNCATCOOH}$ & KRO2HO2*0.820*(1-rchohch2o2_oh) & Rickard and Pascoe \\
\hline Aro481 & TrGCN & $\begin{array}{l}\mathrm{MNNCATECO} 2+\mathrm{HO}_{2} \rightarrow \mathrm{NC} 4 \mathrm{MDCO} 2 \mathrm{HN}+\mathrm{HCOCO}_{2} \mathrm{H} \\
+\mathrm{NO}_{2}+\mathrm{OH}\end{array}$ & KRO2HO2*0.820*rchohch2o2_oh & Rickard and Pascoe (2009)* \\
\hline Aro482 & TrGCN & $\begin{array}{l}\mathrm{MNNCATECO} 2+\mathrm{NO} \rightarrow \mathrm{NC} 4 \mathrm{MDCO} 2 \mathrm{HN}+\mathrm{HCOCO}_{2} \mathrm{H} \\
+\mathrm{NO}_{2}+\mathrm{NO}_{2}\end{array}$ & KRO2NO & Rickard and Pascoe (2009)* \\
\hline Aro483 & TrGCN & $\begin{array}{l}\mathrm{MNNCATECO} 2+\mathrm{NO}_{3} \rightarrow \mathrm{NC} 4 \mathrm{MDCO} 2 \mathrm{HN}+\mathrm{HCOCO}_{2} \mathrm{H} \\
+\mathrm{NO}_{2}+\mathrm{NO}_{2}\end{array}$ & KR02N03 & Rickard and Pascoe $(2009)^{*}$ \\
\hline Aro484 & TrGCN & $\mathrm{MNNCATECO} 2 \rightarrow \mathrm{NC} 4 \mathrm{MDCO} 2 \mathrm{HN}+\mathrm{HCOCO}_{2} \mathrm{H}+\mathrm{NO}_{2}$ & $8.00 \mathrm{E}-13 * \mathrm{R} 02$ & Rickard and Pascoe 2009) \\
\hline Aro485 & TrGCN & $\mathrm{MNCATECO} 2+\mathrm{HO}_{2} \rightarrow$ MNCATECOOH & KRO2HO2*0.820*(1-rchohch2o2_oh) & Rickard and Pascoe (2009) \\
\hline Aro486 & TrGCN & $\begin{array}{l}\mathrm{MNCATECO} 2+\mathrm{HO}_{2} \rightarrow \mathrm{NC} 4 \mathrm{MDCO} 2 \mathrm{HN}+\mathrm{HCOCO}_{2} \mathrm{H} \\
+\mathrm{HO}_{2}+\mathrm{OH}\end{array}$ & KRO2HO2*0.820*rchohch2o2_oh & Rickard and Pascoe (2009)* \\
\hline
\end{tabular}


Table 1: Gas phase reactions (... continued)

\begin{tabular}{|c|c|c|c|c|}
\hline$\#$ & labels & chemical reaction & rate coefficient & reference \\
\hline Aro487 & TrGCN & $\begin{array}{l}\mathrm{MNCATECO} 2+\mathrm{NO} \rightarrow \mathrm{NC} 4 \mathrm{MDCO} 2 \mathrm{HN}+\mathrm{HCOCO}_{2} \mathrm{H}+ \\
\mathrm{HO}_{2}+\mathrm{NO}_{2}\end{array}$ & KRO2NO & Rickard and Pascoe $(\overline{2009})^{*}$ \\
\hline Aro488 & TrGCN & $\begin{array}{l}\mathrm{MNCATECO} 2+\mathrm{NO}_{3} \rightarrow \mathrm{NC} 4 \mathrm{MDCO} 2 \mathrm{HN}+\mathrm{HCOCO}_{2} \mathrm{H} \\
+\mathrm{HO}_{2}+\mathrm{NO}_{2}\end{array}$ & KRO2NO3 & Rickard and Pascoe $(2009)^{*}$ \\
\hline Aro489 & TrGCN & $\mathrm{MNCATECO} 2 \rightarrow \mathrm{NC} 4 \mathrm{MDCO} 2 \mathrm{HN}+\mathrm{HCOCO}_{2} \mathrm{H}+\mathrm{HO}_{2}$ & $8.00 \mathrm{E}-13 * \mathrm{R} 02$ & Rickard and Pascoe 2009)* \\
\hline Aro490 & TrGC & $\mathrm{MCATEC} 1 \mathrm{OOH}+\mathrm{OH} \rightarrow \mathrm{MCATEC} 1 \mathrm{O} 2$ & $2.05 \mathrm{E}-10$ & Rickard and Pascoe $(\overline{2009})$ \\
\hline Aro491 & TrGCN & $\mathrm{C} 3 \mathrm{MCODBPANN}+\mathrm{OH} \rightarrow \mathrm{MGLYOX}+\mathrm{CO}+\mathrm{CO}+\mathrm{NO}_{2}$ & $4.37 \mathrm{E}-11$ & Rickard and Pascoe \\
\hline Aro492 & $\operatorname{TrGCN}$ & $\mathrm{C} 3 \mathrm{MCODBPANN} \rightarrow \mathrm{C} 3 \mathrm{MCODBCO} 3+\mathrm{NO}_{2}$ & k_PAN_M & $\overline{\text { Rickard and Pascoe }}(\overline{\overline{2009}}) *$ \\
\hline Aro493 & TrGC & $\mathrm{MCOCOMOXO} 2+\mathrm{HO}_{2} \rightarrow \mathrm{MCOCOMOOOH}$ & $\mathrm{KRO} 2 \mathrm{HO} 2 * 0.625 *(1-.4)$ & $\overline{\text { Rickard and Pascoe }}(\overline{2009})^{*}$ \\
\hline Aro494 & TrGC & $\mathrm{MCOCOMOXO} 2+\mathrm{HO}_{2} \rightarrow \mathrm{HCHO}+\mathrm{CH}_{3} \mathrm{C}(\mathrm{O}) \mathrm{OO}+\mathrm{OH}$ & $\mathrm{KRO} 2 \mathrm{HO} 2 * 0.625 * .4$ & Rickard and Pascoe $(2009)^{*}$ \\
\hline Aro495 & TrGCN & $\mathrm{MCOCOMOXO} 2+\mathrm{NO} \rightarrow \mathrm{HCHO}+\mathrm{CH}_{3} \mathrm{C}(\mathrm{O}) \mathrm{OO}+\mathrm{NO}_{2}$ & KRO2NO & Rickard and Pascoe $(2009)^{*}$ \\
\hline Aro496 & TrGCN & $\mathrm{MCOCOMOXO} 2+\mathrm{NO}_{3} \rightarrow \mathrm{HCHO}+\mathrm{CH}_{3} \mathrm{C}(\mathrm{O}) \mathrm{OO}+\mathrm{NO}_{2}$ & KR02NO3 & Rickard and Pascoe $(\overline{\overline{2009}})^{*}$ \\
\hline Aro497 & TrGC & $\mathrm{MCOCOMOXO} 2 \rightarrow \mathrm{HCHO}+\mathrm{CH}_{3} \mathrm{C}(\mathrm{O}) \mathrm{OO}$ & KRO2pOR02*R02 & Rickard and Pascoe $(\overline{2009})^{*}$ \\
\hline Aro498 & TrGCN & $\mathrm{NPTLQOOH}+\mathrm{OH} \rightarrow \mathrm{NPTLQO} 2$ & $8.56 \mathrm{E}-11$ & Rickard and Pascoe (2009) \\
\hline Aro499 & TrGC & $\mathrm{PTLQOOH}+\mathrm{OH} \rightarrow \mathrm{PTLQCO}+\mathrm{OH}$ & $1.42 \mathrm{E}-10$ & Rickard and Pascoe (2009) \\
\hline Aro500 & TrGC & $\mathrm{PTLQCO}+\mathrm{OH} \rightarrow \mathrm{C} 6 \mathrm{CO} 2 \mathrm{OHCO} 3$ & $7.95 \mathrm{E}-11$ & 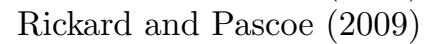 \\
\hline Aro501 & TrGCN & $\mathrm{NDNCRESO} 2+\mathrm{HO}_{2} \rightarrow \mathrm{NDNCRESOOH}$ & KRO2HO2*0.820*(1-rchohch2o2_oh) & Rickard and Pascoe $(\overline{2009})$ \\
\hline Aro502 & TrGCN & $\begin{array}{l}\mathrm{NDNCRESO} 2+\mathrm{HO}_{2} \rightarrow \mathrm{NC} 4 \mathrm{MDCO} 2 \mathrm{HN}+\mathrm{HNO}_{3}+\mathrm{CO} \\
+\mathrm{CO}+\mathrm{NO}_{2}+\mathrm{OH}\end{array}$ & KRO2HO2*0.820*rchohch2o2_oh & Rickard and Pascoe $(2009)^{*}$ \\
\hline Aro503 & TrGCN & $\begin{array}{l}\mathrm{NDNCRESO} 2+\mathrm{NO} \rightarrow \mathrm{NC} 4 \mathrm{MDCO} 2 \mathrm{HN}+\mathrm{HNO}_{3}+\mathrm{CO} \\
+\mathrm{CO}+\mathrm{NO}_{2}+\mathrm{NO}_{2}\end{array}$ & KRO2NO & Rickard and Pascoe $(2009)^{*}$ \\
\hline Aro504 & TrGCN & $\begin{array}{l}\mathrm{NDNCRESO} 2+\mathrm{NO}_{3} \rightarrow \mathrm{NC} 4 \mathrm{MDCO} 2 \mathrm{HN}+\mathrm{HNO}_{3}+\mathrm{CO} \\
+\mathrm{CO}+\mathrm{NO}_{2}+\mathrm{NO}_{2}\end{array}$ & KR02NO3 & Rickard and Pascoe $(2009)^{*}$ \\
\hline Aro505 & TrGCN & $\begin{array}{l}\mathrm{NDNCRESO} 2 \rightarrow \mathrm{NC} 4 \mathrm{MDCO} 2 \mathrm{HN}+\mathrm{HNO}_{3}+\mathrm{CO}+\mathrm{CO} \\
+\mathrm{NO}_{2}\end{array}$ & $8.00 \mathrm{E}-13 * \mathrm{R} 02$ & Rickard and Pascoe $(2009)^{*}$ \\
\hline Aro506 & TrGCN & DNCRESO $2+\mathrm{HO}_{2} \rightarrow$ DNCRESOOH & KRO2HO2*0.820*(1-rchohch2o2_oh) & Rickard and Pascoe (2009) \\
\hline Aro507 & TrGCN & $\begin{array}{l}\mathrm{DNCRESO} 2+\mathrm{HO}_{2} \rightarrow \mathrm{NC} 4 \mathrm{MDCO} 2 \mathrm{HN}+\mathrm{HCOCO}_{2} \mathrm{H}+ \\
\mathrm{NO}_{2}+\mathrm{OH}\end{array}$ & KRO2HO2*0.820*rchohch2o2_oh & Rickard and Pascoe $(2009)^{*}$ \\
\hline Aro508 & TrGCN & $\begin{array}{l}\mathrm{DNCRESO} 2 \\
\mathrm{NO}_{2}+\mathrm{NO} \mathrm{NO}_{2}\end{array}$ & KRO2NO & Rickard and Pascoe $(2009)^{*}$ \\
\hline Aro509 & TrGCN & $\begin{array}{l}\mathrm{DNCRESO} 2+\mathrm{NO}_{3} \rightarrow \mathrm{NC} 4 \mathrm{MDCO} 2 \mathrm{HN}+\mathrm{HCOCO}_{2} \mathrm{H}+ \\
\mathrm{NO}_{2}+\mathrm{NO}_{2}\end{array}$ & KR02NO3 & Rickard and Pascoe $(2009)^{*}$ \\
\hline Aro510 & $\operatorname{TrGCN}$ & $\mathrm{DNCRESO} 2 \rightarrow \mathrm{NC} 4 \mathrm{MDCO} 2 \mathrm{HN}+\mathrm{HCOCO}_{2} \mathrm{H}+\mathrm{NO}_{2}$ & $8.00 \mathrm{E}-13 * \mathrm{R} 02$ & Rickard and Pascoe (2009)* \\
\hline Aro511 & TrGCN & $\mathrm{NCRES} 1 \mathrm{OOH}+\mathrm{OH} \rightarrow \mathrm{NCRES} 1 \mathrm{O} 2$ & $1.53 \mathrm{E}-12$ & Rickard and Pascoe (2009) \\
\hline Aro512 & TrGC & $\mathrm{MNNCATCOOH}+\mathrm{OH} \rightarrow$ MNNCATECO2 & $1.90 \mathrm{E}-12 * \operatorname{EXP}(190 / \mathrm{TEMP})$ & Rickard and Pascoe (2009) \\
\hline
\end{tabular}


Table 1: Gas phase reactions (... continued)

\begin{tabular}{|c|c|c|c|c|}
\hline \# & labels & chemical reaction & rate coefficient & reference \\
\hline Aro513 & TrGCN & MNCATECOOH $+\mathrm{OH} \rightarrow$ MNCATECO2 & 1.90E-12*EXP(190/TEMP) & Rickard and Pascoe $(2009)$ \\
\hline Aro514 & TrGCN & $\begin{array}{l}\mathrm{NO}_{3}+\mathrm{CO} 23 \mathrm{C} 3 \mathrm{CHO} \rightarrow \mathrm{CH}_{3} \mathrm{C}(\mathrm{O}) \mathrm{OO}+\mathrm{CO}+\mathrm{CO}+ \\
\mathrm{HNO}_{3}\end{array}$ & KNO3AL $* 4.0$ & $\overline{\overline{\text { Rickard and Pascoe }}}(\overline{\overline{2009}})$ \\
\hline Aro515 & $\operatorname{TrGC}$ & $\mathrm{OH}+\mathrm{CO} 23 \mathrm{C} 3 \mathrm{CHO} \rightarrow \mathrm{CH}_{3} \mathrm{C}(\mathrm{O}) \mathrm{OO}+\mathrm{CO}+\mathrm{CO}$ & $1.23 \mathrm{E}-11$ & Rickard and Pascoe (2009) \\
\hline Aro516 & $\operatorname{TrGC}$ & $\mathrm{C} 7 \mathrm{CO} 4 \mathrm{DB}+\mathrm{OH} \rightarrow \mathrm{CO}+\mathrm{CO}+\mathrm{CH}_{3} \mathrm{C}(\mathrm{O}) \mathrm{OO}+\mathrm{C} 33 \mathrm{CO}$ & $9.58 \mathrm{E}-11$ & $\overline{\text { Rickard and Pascoe }}(\overline{\overline{2009}})$ \\
\hline Aro517 & $\operatorname{TrGC}$ & $\begin{array}{l}\mathrm{C} 6 \mathrm{CO} 2 \mathrm{OHCO} 3+\mathrm{HO}_{2} \rightarrow \mathrm{C} 5134 \mathrm{CO} 2 \mathrm{OH}+\mathrm{HO}_{2}+\mathrm{CO}+ \\
\mathrm{OH}\end{array}$ & KAPHO2*rco3_oh & $\overline{\overline{\text { Rickard and Pascoe }}}(\overline{\overline{2009}})^{*}$ \\
\hline Aro518 & TrGC & $\mathrm{C} 6 \mathrm{CO} 2 \mathrm{OHCO} 3+\mathrm{HO}_{2} \rightarrow \mathrm{C} 6 \mathrm{COOHCO} 3 \mathrm{H}$ & KAPHO2 $*\left(r c 03 \_o o h+r c 03 \_o 3\right)$ & Rickard and Pascoe $(2009)^{*}$ \\
\hline Aro519 & TrGCN & $\begin{array}{l}\mathrm{C} 6 \mathrm{CO} 2 \mathrm{OHCO} 3+\mathrm{NO} \rightarrow \mathrm{C} 5134 \mathrm{CO} 2 \mathrm{OH}+\mathrm{HO}_{2}+\mathrm{CO}+ \\
\mathrm{NO}_{2}\end{array}$ & KAPNO & Rickard and Pascoe $(\overline{2009})$ \\
\hline Aro520 & $\operatorname{TrGCN}$ & $\mathrm{C} 6 \mathrm{CO} 2 \mathrm{OHCO} 3+\mathrm{NO}_{2} \rightarrow \mathrm{C} 6 \mathrm{CO} 2 \mathrm{OHPAN}$ & k_CH3CO3_NO2 & Rickard and Pascoe $(2009)^{*}$ \\
\hline Aro521 & TrGCN & $\begin{array}{l}\mathrm{C} 6 \mathrm{CO} 2 \mathrm{OHCO} 3+\mathrm{NO}_{3} \rightarrow \mathrm{C} 5134 \mathrm{CO} 2 \mathrm{OH}+\mathrm{HO}_{2}+\mathrm{CO}+ \\
\mathrm{NO}_{2}\end{array}$ & KRO2NO3*1.74 & $\overline{\text { Rickard and Pascoe }}(\overline{\overline{2009}})$ \\
\hline Aro522 & $\operatorname{TrGC}$ & $\mathrm{C} 6 \mathrm{CO} 2 \mathrm{OHCO} 3 \rightarrow \mathrm{C} 5134 \mathrm{CO} 2 \mathrm{OH}+\mathrm{HO}_{2}+\mathrm{CO}$ & $\mathrm{KRO} 2 \mathrm{AP} * \mathrm{RO} 2$ & Rickard and Pascoe (2009) \\
\hline Aro523 & TrGCN & $\mathrm{NDNCRESOOH}+\mathrm{OH} \rightarrow$ NDNCRESO2 & $1.90 \mathrm{E}-12 * \operatorname{EXP}(190 / \mathrm{TEMP})$ & $\overline{\text { Rickard and Pascoe }}(\overline{\overline{2009}})$ \\
\hline Aro524 & TrGCN & DNCRESOOH $+\mathrm{OH} \rightarrow$ DNCRESO2 & 1.90E-12*EXP(190/TEMP) & Rickard and Pascoe $(\overline{\overline{2009}})$ \\
\hline Aro525 & TrGC & $\mathrm{C} 6 \mathrm{COOHCO} 3 \mathrm{H}+\mathrm{OH} \rightarrow \mathrm{C} 6 \mathrm{CO} 2 \mathrm{OHCO} 3$ & $9.29 \mathrm{E}-11$ & $\overline{\text { Rickard and Pascoe }}(\overline{\overline{2009}})$ \\
\hline Aro526 & TrGCN & $\begin{array}{l}\mathrm{C} 6 \mathrm{CO} 2 \mathrm{OHPAN}+\mathrm{OH} \rightarrow \mathrm{C} 5134 \mathrm{CO} 2 \mathrm{OH}+\mathrm{CO}+\mathrm{CO}+ \\
\mathrm{NO}_{2}\end{array}$ & $8.96 \mathrm{E}-11$ & 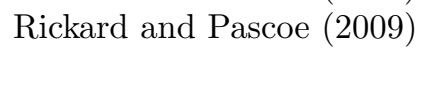 \\
\hline Aro527 & TrGCN & $\mathrm{C} 6 \mathrm{CO} 2 \mathrm{OHPAN} \rightarrow \mathrm{C} 6 \mathrm{CO} 2 \mathrm{OHCO} 3+\mathrm{NO}_{2}$ & k_PAN_M & Rickard and Pascoe $(2009)^{*}$ \\
\hline Aro528 & TrGC & $\begin{array}{l}\mathrm{LXYL}+\mathrm{OH} \rightarrow \underset{\mathrm{TLEPOXMUC}}{\rightarrow}+\mathrm{HO}_{2}+ \\
\text { PXYL }_{\mathrm{T}} \text { LEPOXMUC }+ \text { LCARBON }\end{array}$ & $0.401 \mathrm{E}-11$ & Rickard and Pascoe $(\overline{2009})^{*}$ \\
\hline Aro529 & TrGC & $\begin{array}{l}\mathrm{LXYL}+\mathrm{OH} \rightarrow \mathrm{C} 6 \mathrm{H} 5 \mathrm{CH} 2 \mathrm{O} 2+\mathrm{PXYL}_{\mathrm{C}} 6 \mathrm{H} 5 \mathrm{CH} 2 \mathrm{O} 2+ \\
\text { LCARBON }\end{array}$ & $0.101 \mathrm{E}-11$ & Rickard and Pascoe $(2009)^{*}$ \\
\hline Aro530 & TrGC & $\mathrm{LXYL}+\mathrm{OH} \rightarrow \mathrm{CRESOL}+\mathrm{PXYL}_{\mathrm{C}} \mathrm{RESOL}+\mathrm{LCARBON}$ & $0.261 \mathrm{E}-11$ & Rickard and Pascoe (2009)* \\
\hline Aro531 & $\operatorname{TrGC}$ & $\begin{array}{l}\mathrm{LXYL}+\mathrm{OH} \rightarrow \mathrm{TLBIPERO} 2+\mathrm{HO}_{2}+\mathrm{PXYL}_{\mathrm{T}} \mathrm{LBIPERO} 2 \\
+\mathrm{LCARBON}\end{array}$ & $0.932 \mathrm{E}-11$ & 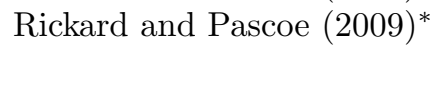 \\
\hline Aro532 & $\operatorname{TrGCN}$ & $\begin{array}{l}\mathrm{LXYL}+\mathrm{NO}_{3} \rightarrow \underset{\mathrm{C} 6 \mathrm{H} 5 \mathrm{CH} 2 \mathrm{O} 2}{\rightarrow}+\mathrm{HNO}_{3}+ \\
\text { PXYL }_{\mathrm{C}} 6 \mathrm{H} 5 \mathrm{CH} 2 \mathrm{O} 2+\mathrm{LCARBON}\end{array}$ & $3.9 \mathrm{E}-16$ & Rickard and Pascoe $(2009)^{*}$ \\
\hline Aro533 & TrGC & $\begin{array}{l}\mathrm{LTMB}+\mathrm{OH} \rightarrow \underset{\mathrm{TLEPOXMUC}}{\rightarrow}+\underset{\mathrm{HO}_{2}}{\text { TLMB }}+ \\
\mathrm{PTEPOXMUC}_{\mathrm{T}} \text { LCARBON }\end{array}$ & $0.827 \mathrm{E}-11$ & Rickard and Pascoe $(2009)^{*}$ \\
\hline Aro534 & TrGC & $\begin{array}{l}\mathrm{LTMB}+\mathrm{OH} \rightarrow \mathrm{C} 6 \mathrm{H} 5 \mathrm{CH} 2 \mathrm{O} 2+\mathrm{PTMB}_{\mathrm{C}} 6 \mathrm{H} 5 \mathrm{CH} 2 \mathrm{O} 2+2 \\
\mathrm{LCARBON}\end{array}$ & $0.189 \mathrm{E}-11$ & Rickard and Pascoe $(2009)^{*}$ \\
\hline Aro535 & TrGC & $\begin{array}{l}\mathrm{LTMB}+\mathrm{OH} \\
\mathrm{LCARBON}\end{array}$ & $0.141 \mathrm{E}-11$ & Rickard and Pascoe $(2009) *$ \\
\hline
\end{tabular}


Table 1: Gas phase reactions (... continued)

\begin{tabular}{|c|c|c|c|c|}
\hline \# & labels & chemical reaction & rate coefficient & reference \\
\hline Aro536 & $\operatorname{TrGC}$ & 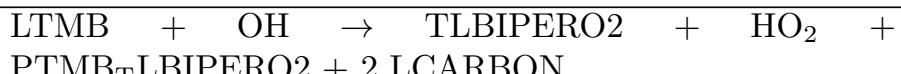 & $2.917 \mathrm{E}-11$ & $\overline{\text { Rickard and Pascoe }}(\overline{2009})^{*}$ \\
\hline Aro537 & TrGCN & $\begin{array}{l}\mathrm{LTMB}+\mathrm{NO}_{3} \rightarrow \underset{\mathrm{C} 6 \mathrm{H} 5 \mathrm{CH} 2 \mathrm{O} 2}{\mathrm{PTMB}_{\mathrm{C}} 6 \mathrm{H} 5 \mathrm{CH} 2 \mathrm{O} 2+2 \mathrm{LCARBON}} \\
+\mathrm{HNO}_{3}+\end{array}$ & $1.52 \mathrm{E}-15$ & Rickard and Pascoe $(2009)^{*}$ \\
\hline Aro538 & $\operatorname{TrGC}$ & $\begin{array}{l}\mathrm{EBENZ}+\mathrm{OH} \rightarrow .10 \text { TLEPOXMUC }+.07 \mathrm{C} 6 \mathrm{H} 5 \mathrm{CH} 2 \mathrm{O} 2+ \\
.18 \mathrm{CRESOL}+.65 \text { TLBIPERO } 2+0.28 \mathrm{HO}_{2}+\mathrm{LCARBON}\end{array}$ & $7.00 \mathrm{E}-12$ & Rickard and Pascoe $(2009)^{*}$ \\
\hline Aro539 & TrGCN & $\mathrm{EBENZ}+\mathrm{NO}_{3} \rightarrow \mathrm{C} 6 \mathrm{H} 5 \mathrm{CH} 2 \mathrm{O} 2+\mathrm{HNO}_{3}+\mathrm{LCARBON}$ & $1.20 \mathrm{E}-16$ & Rickard and Pascoe $(2009)^{*}$ \\
\hline Aro540 & $\operatorname{TrGC}$ & $\begin{array}{l}\mathrm{HAROM}+\mathrm{OH} \rightarrow .14 \text { TLEPOXMUC }+.03 \mathrm{C} 6 \mathrm{H} 5 \mathrm{CH} 2 \mathrm{O} 2 \\
+.04 \text { CRESOL }+.79 \text { TLBIPERO } 2+0.18 \mathrm{HO}_{2}+4 \\
\text { LCARBON }\end{array}$ & $5.67 \mathrm{E}-11$ & $\overline{\text { Rickard and Pascoe }}(\overline{\overline{2009}})^{*}$ \\
\hline Aro541 & $\operatorname{TrGCN}$ & $\mathrm{HAROM}+\mathrm{NO}_{3} \rightarrow \mathrm{C} 6 \mathrm{H} 5 \mathrm{CH} 2 \mathrm{O} 2+\mathrm{HNO}_{3}+4 \mathrm{LCARBON}$ & $2.60 \mathrm{E}-15$ & Rickard and Pascoe $(2009)^{*}$ \\
\hline Aro542 & TrGCN & $\mathrm{NO}_{3}+\mathrm{STYRENE} \rightarrow \mathrm{NSTYRENO} 2$ & $1.50 \mathrm{E}-12$ & Rickard and Pascoe (2009) \\
\hline Aro543 & $\operatorname{TrGC}$ & $\begin{array}{l}\mathrm{O}_{3}+\mathrm{STYRENE} \rightarrow .545 \mathrm{HCHO}+.1 \mathrm{BENZENE}+.28 \\
\mathrm{C} 6 \mathrm{H} 5 \mathrm{O} 2+.56 \mathrm{CO}+.36 \mathrm{OH}+.28 \mathrm{HO}_{2}+.075 \mathrm{PHCOOH} \\
+.545 \mathrm{BENZAL}+.09 \mathrm{H}_{2} \mathrm{O}_{2}+.075 \mathrm{HCOOH}\end{array}$ & $1.70 \mathrm{E}-17$ & Rickard and Pascoe (2009)* \\
\hline Aro544 & TrGC & $\mathrm{OH}+\mathrm{STYRENE} \rightarrow \mathrm{STYRENO} 2$ & $5.80 \mathrm{E}-11$ & Rickard and Pascoe (2009) \\
\hline Aro545 & TrGCN & $\mathrm{NSTYRENO} 2+\mathrm{HO}_{2} \rightarrow$ NSTYRENOOH & KRO2HO2 $* 0.859$ & Rickard and Pascoe $(\overline{2009})$ \\
\hline Aro546 & TrGCN & $\mathrm{NSTYRENO} 2+\mathrm{NO} \rightarrow \mathrm{NO}_{2}+\mathrm{NO}_{2}+\mathrm{HCHO}+\mathrm{BENZAL}$ & KRO2NO & Rickard and Pascoe $(\overline{\overline{2009}})^{*}$ \\
\hline Aro547 & TrGCN & $\begin{array}{l}\text { NSTYRENO2 } \\
\text { BENZAL }\end{array}$ & KRO2NO3 & Rickard and Pascoe $(\overline{2009})^{*}$ \\
\hline Aro548 & $\operatorname{TrGCN}$ & NSTYRENO $2 \rightarrow \mathrm{NO}_{2}+\mathrm{HCHO}+\mathrm{BENZAL}$ & KRO2sR02*R02 & Rickard and Pascoe (2009)* \\
\hline Aro549 & TrGCN & $\mathrm{OH}+\mathrm{NSTYRENOOH} \rightarrow$ NSTYRENO2 & $6.16 \mathrm{E}-11$ & Rickard and Pascoe $(\overline{2009})$ \\
\hline Aro550 & TrGC & $\mathrm{STYRENO} 2+\mathrm{HO}_{2} \rightarrow \mathrm{STYRENOOH}$ & KRO2HO2 $* 0.859 *\left(1-r c h o h c h 2 \circ 2 \_o h\right)$ & Rickard and Pascoe (2009) \\
\hline Aro551 & TrGCN & $\mathrm{STYRENO} 2+\mathrm{HO}_{2} \rightarrow \mathrm{NO}_{2}+\mathrm{OH}+\mathrm{HCHO}+\mathrm{BENZAL}$ & KRO2HO2*0.859*rchohch2o2_oh & Rickard and Pascoe $(\overline{2009})^{*}$ \\
\hline Aro552 & TrGCN & $\mathrm{STYRENO} 2+\mathrm{NO} \rightarrow \mathrm{NO}_{2}+\mathrm{HO}_{2}+\mathrm{HCHO}+\mathrm{BENZAL}$ & KRO2NO & $\overline{\text { Rickard and Pascoe }}(\overline{\overline{2009}})^{*}$ \\
\hline Aro553 & TrGCN & $\mathrm{STYRENO} 2+\mathrm{NO}_{3} \rightarrow \mathrm{NO}_{2}+\mathrm{HO}_{2}+\mathrm{HCHO}+\mathrm{BENZAL}$ & KR02NO3 & Rickard and Pascoe $2 \overline{2009})^{*}$ \\
\hline Aro554 & $\operatorname{TrGC}$ & $\mathrm{STYRENO} 2 \rightarrow \mathrm{HO}_{2}+\mathrm{HCHO}+\mathrm{BENZAL}$ & KRO2sR02*R02 & Rickard and Pascoe $(\overline{\overline{2009}})^{*}$ \\
\hline Aro555 & $\operatorname{TrGC}$ & $\mathrm{OH}+\mathrm{STYRENOOH} \rightarrow \mathrm{STYRENO} 2$ & $6.16 \mathrm{E}-11$ & $\overline{\text { Rickard and Pascoe }}(\overline{\overline{2009}})$ \\
\hline
\end{tabular}

Aromatic gas phase reactions notes:

Aro003: MGLYOX + GLYOX + HO2 from KDEC substitution

Aro004: MGLYOX + GLYOX + HO2 from KDEC substitution $\quad \mathrm{HO} 2$

Aro005: permutation reaction (minor channels re- Aro012: KDEC C3DIALO $\rightarrow$ GLYOX + CO + HO2 moved)

Aro011: $\quad$ KDEC C3DIALO $\rightarrow$ GLYOX + CO + Aro014: rco3_oh updated 
Aro015: rco3_ooh+rco3_o3 updated

Aro029: $\mathrm{KDEC}$ NC4DCO2 $\rightarrow$ MALANHY + NO2

Aro033: rco3_o3 updated

Aro034: rco3_ooh updated

Aro035: rco3_oh updated + KDEC MALDIALCO2

$\rightarrow .6 \mathrm{MALANHY}+\mathrm{HO} 2+.4 \mathrm{GLYOX}+.4 \mathrm{CO}$

Aro036: KDEC MALDIALCO2 $\rightarrow .6$ MALANHY + $\mathrm{HO} 2+.4$ GLYOX $+.4 \mathrm{CO}$

Aro038: KDEC MALDIALCO2 $\rightarrow .6$ MALANHY + $\mathrm{HO} 2+.4$ GLYOX $+.4 \mathrm{CO}$

Aro039: KDEC MALDIALCO2 $\rightarrow .6$ MALANHY + $\mathrm{HO} 2+.4$ GLYOX $+.4 \mathrm{CO}$

Aro041: KDEC BZFUONOOA $\rightarrow .5$ BZFUONOO + $.5 \mathrm{CO}+.5 \mathrm{HCOCH} 2 \mathrm{O} 2+.5 \mathrm{OH}$ and $\mathrm{BZFUONOO} \rightarrow$ $.625 \mathrm{CO} 14 \mathrm{O} 3 \mathrm{CO} 2 \mathrm{H}+.375 \mathrm{CO} 14 \mathrm{O} 3 \mathrm{CHO}+.375 \mathrm{H} 2 \mathrm{O} 2$ Aro046: updated rco3_oh

Aro047: updated rco3_o3

Aro048: updated rco3_ooh

Aro052: Only major channel taken

Aro055: KDEC: GLYOOA $\rightarrow .125 \mathrm{HCHO}+.18$

GLYOO + $0.82 \mathrm{HO} 2+.57 \mathrm{OH}+1.265 \mathrm{CO}$ and $\mathrm{H} 2 \mathrm{O}$ substitution GLYOO $\rightarrow .625 \mathrm{HCOCO} 2 \mathrm{H}+.375$ GLYOX + .375 H2O2

Aro056: merged equations

Aro062: KDEC MALANHYO $\rightarrow \mathrm{HCOCOHCO3}$

Aro063: KDEC MALANHYO $\rightarrow$ HCOCOHCO3

Aro064: Only major channel taken and KDEC

$\mathrm{MALANHYO} \rightarrow \mathrm{HCOCOHCO} 3$

Aro069: KDEC NBZFUO $\rightarrow .5 \mathrm{CO} 14 \mathrm{O} 3 \mathrm{CHO}+$ $.5 \mathrm{NO} 2+.5 \mathrm{NBZFUONE}+.5 \mathrm{HO} 2$

Aro070: KDEC NBZFUO $\rightarrow .5 \mathrm{CO} 14 \mathrm{O} 3 \mathrm{CHO}+.5$ $\mathrm{NO} 2+.5 \mathrm{NBZFUONE}+.5 \mathrm{HO} 2$

Aro071: KDEC NBZFUO $\rightarrow .5 \mathrm{CO} 14 \mathrm{O} 3 \mathrm{CHO}+.5$

$\mathrm{NO} 2+.5 \mathrm{NBZFUONE}+.5 \mathrm{HO} 2$ and RO 2 Only major channel taken

Aro072: KDEC MALDIALCO2 $\rightarrow .6$ MALANHY +
$\mathrm{HO} 2+.4$ GLYOX $+.4 \mathrm{CO}$

Aro077: KDEC MECOACETO $\rightarrow \mathrm{CH} 3 \mathrm{CO} 3+\mathrm{HCHO}$

Aro078: $\mathrm{KDEC} \mathrm{MECOACETO} \rightarrow \mathrm{CH} 3 \mathrm{CO} 3+\mathrm{HCHO}$

Aro079: KDEC MECOACETO $\rightarrow \mathrm{CH} 3 \mathrm{CO} 3+\mathrm{HCHO}$

Aro085: $\quad$ KDEC BZFUO $\rightarrow \mathrm{CO} 14 \mathrm{O} 3 \mathrm{CHO}+\mathrm{HO} 2$

Aro086: $\mathrm{KDEC}$ BZFUO $\rightarrow \mathrm{CO} 14 \mathrm{O} 3 \mathrm{CHO}+\mathrm{HO} 2$

Aro087: KDEC BZFUO $\rightarrow \mathrm{CO} 14 \mathrm{O} 3 \mathrm{CHO}+\mathrm{HO} 2$ and

Only major channel taken

Aro093: $\quad$ KDEC MALDIALO $\rightarrow$ GLYOX + GLYOX

$+\mathrm{HO} 2$

Aro094: KDEC MALDIALO $\rightarrow$ GLYOX + GLYOX

$+\mathrm{HO} 2$

Aro095: KDEC MALDIALO $\rightarrow$ GLYOX + GLYOX

$+\mathrm{HO} 2$ and Only major channel taken

Aro096: KDEC NC4MDCO2 $\rightarrow$ MMALANHY + NO2

Aro101: KDEC NTLFUO $\rightarrow$ ACCOMECHO + NO2

Aro102: $\quad$ KDEC NTLFUO $\rightarrow$ ACCOMECHO + NO2

Aro103: KDEC NTLFUO $\rightarrow$ ACCOMECHO

Aro105: KDEC MC3CODBCO2 $\rightarrow .35$ GLYOX +.35

$\mathrm{CH} 3 \mathrm{O} 2+.35 \mathrm{CO}+.65 \mathrm{MMALANHY}+.65 \mathrm{HO} 2$ and

updated rco3_oh

Aro106: updated rco3_o3

Aro107: updated rco3_ooh

Aro108: KDEC MC3CODBCO2 $\rightarrow .35$ GLYOX +.35 $\mathrm{CH} 3 \mathrm{O} 2+.35 \mathrm{CO}+.65 \mathrm{MMALANHY}+.65 \mathrm{HO} 2$

Aro110: KDEC MC3CODBCO2 $\rightarrow .35$ GLYOX +.35 $\mathrm{CH} 3 \mathrm{O} 2+.35 \mathrm{CO}+.65$ MMALANHY + .65 HO 2

Aro111: KDEC MC3CODBCO2 $\rightarrow .35$ GLYOX +.35 $\mathrm{CH} 3 \mathrm{O} 2+.35 \mathrm{CO}+.65$ MMALANHY $+.65 \mathrm{HO} 2$ and Only major channel taken

Aro116: updated rco3_ooh+rco3_o3

Aro117: updated rco3_oh
Aro125: KDEC NPXYFUO $\rightarrow \mathrm{C} 23 \mathrm{O} 3 \mathrm{CCHO}+\mathrm{NO} 2$

Aro126: $\mathrm{KDEC}$ NPXYFUO $\rightarrow \mathrm{C} 23 \mathrm{O} 3 \mathrm{CCHO}+\mathrm{NO} 2$

Aro127: KDEC NPXYFUO $\rightarrow \mathrm{C} 23 \mathrm{O} 3 \mathrm{CCHO}+\mathrm{NO} 2$

Aro130: KDEC MMALANHYO $\rightarrow \mathrm{CO} 2 \mathrm{H} 3 \mathrm{CO} 3$

Aro131: KDEC MMALANHYO $\rightarrow \mathrm{CO} 2 \mathrm{H} 3 \mathrm{CO} 3$

Aro132: $\quad$ KDEC MMALANHYO $\rightarrow \mathrm{CO} 2 \mathrm{H} 3 \mathrm{CO} 3$ and Only major channel taken

Aro138: updated rco3_oh and KDEC C5CO14CO2 $\rightarrow .83$ MALANHY + .83 CH3O2 + .17 MGLYOX + $.17 \mathrm{HO} 2+.17 \mathrm{CO}$

Aro139: updated rco3 o3

Aro140: updated rco3_ooh

Aro141: KDEC C5CO14CO2 $\rightarrow .83 \mathrm{MAIANHY}+.83$ $\mathrm{CH} 3 \mathrm{O} 2+.17 \mathrm{MGLYOX}+.17 \mathrm{HO} 2+.17 \mathrm{CO}$

Aro143: KDEC C5CO14CO2 $\rightarrow .83$ MALANHY +.83 $\mathrm{CH} 3 \mathrm{O} 2+.17 \mathrm{MGLYOX}+.17 \mathrm{HO} 2+.17 \mathrm{CO}$

Aro144: KDEC C5CO14CO2 $\rightarrow .83$ MALANHY + $.83 \mathrm{CH} 3 \mathrm{O} 2+.17 \mathrm{MGLYOX}+.17 \mathrm{HO} 2+.17 \mathrm{CO}$ and Only major channel taken

Aro148: $\quad$ KDEC C5CO14CO2 $\rightarrow .83$ MALANHY + $.83 \mathrm{CH} 3 \mathrm{O} 2+.17 \mathrm{MGLYOX}+.17 \mathrm{HO} 2+.17 \mathrm{CO}$

Aro151: KDEC NPXYFUO $\rightarrow \mathrm{C} 23 \mathrm{O} 3 \mathrm{CCHO}+\mathrm{NO} 2$

Aro152: KDEC NPXYFUO $\rightarrow \mathrm{C} 23 \mathrm{O} 3 \mathrm{CCHO}+\mathrm{NO} 2$ Aro153: KDEC NPXYFUO $\rightarrow \mathrm{C} 23 \mathrm{O} 3 \mathrm{CCHO}+\mathrm{NO} 2$ $/ / 1$ original $/ / \mathrm{C} 5 \mathrm{DICARB}+\mathrm{O} 3=\mathrm{GLYOX}+$ MGLOOB : $2.00 \mathrm{E}-18^{*} 0.5$;

$/ / 1 . \quad \mathrm{KDEC}$ C5DICARB $+\mathrm{O} 3=\mathrm{GLYOX}+.125$ $\mathrm{CH} 3 \mathrm{CHO}+.695 \mathrm{CH} 3 \mathrm{CO} 3+.57 \mathrm{CO}+.57 \mathrm{OH}+.125$ $\mathrm{HO} 2+.18 \mathrm{MGLOO}: 2.00 \mathrm{E}-18^{*} 0.5$;

$/ / 2$. original $/ / \mathrm{C} 5 \mathrm{DICARB}+\mathrm{O} 3=$ MGLYOX + GLYOOA : $2.00 \mathrm{E}-18^{*} 0.5$;

$/ / 2$. $\mathrm{KDEC}$ C5DICARB $+\mathrm{O} 3=\mathrm{MGLYOX}+.125$ $\mathrm{HCHO}+.18 \mathrm{GLYOO}+0.82 \mathrm{HO} 2+.57 \mathrm{OH}+1.265$ $\mathrm{CO}: 2.00 \mathrm{E}-18^{*} 0.5$;

$/ / 3$. All merged C5DICARB + O3 $=.5$ GLYOX +.063 $\mathrm{CH} 3 \mathrm{CHO}+.348 \mathrm{CH} 3 \mathrm{CO} 3+.285 \mathrm{CO}+.285 \mathrm{OH}+$ 
$.063 \mathrm{HO} 2+.09 \mathrm{MGLOO}+.5 \mathrm{MGLYOX}+.063 \mathrm{HCHO}$ $+.09 \mathrm{GLYOO}+0.41 \mathrm{HO} 2+.285 \mathrm{OH}+.633 \mathrm{CO}$ Aro157: KDEC MC3CODBCO2 $\rightarrow .35$ GLYOX +.35 $\mathrm{CH} 3 \mathrm{O} 2+.35 \mathrm{CO}+.65 \mathrm{MMALANHY}+.65 \mathrm{HO} 2$

Aro159: updated rco3_o3

Aro160: updated rco3_ooh

Aro161: updated rco3_oh

Aro165: Only major channel taken

Aro167: KDEC TLFUONOOA $\rightarrow .5 \mathrm{CO}+.5 \mathrm{OH}+$ .5 MECOACETO2 + .5 TLFUONOO and H2Osubs TLFUONOO $\rightarrow .625 \mathrm{C} 24 \mathrm{O} 3 \mathrm{CCO} 2 \mathrm{H}+.375$ ACCOME$\mathrm{CHO}+.375 \mathrm{H} 2 \mathrm{O} 2$

Aro169: updated rco3_ooh+rco3_o3

Aro170: updated rco3_oh

Aro180: $\quad$ KDEC C4M2ALOHO $\rightarrow$ GLYOX + MG$\mathrm{LYOX}+\mathrm{HO} 2$

Aro181: $\quad$ KDEC C4M2ALOHO $\rightarrow$ GLYOX + MG-

$\mathrm{LYOX}+\mathrm{HO} 2$

Aro182: Only major channel taken

Aro193: $\quad$ KDEC C4M2ALOHO2 $\rightarrow$ GLYOX + MG$\mathrm{LYOX}+\mathrm{HO} 2$

Aro194: updated rco3_oh and KDEC $\mathrm{C} 3 \mathrm{MCODBCO} 2 \rightarrow .35 \mathrm{MGLYOX}+.35 \mathrm{HO} 2+.35$ $\mathrm{CO}+.65$ MMALANHY $+.65 \mathrm{HO} 2$

Aro195: updated rco3_ooh+rco3_o3

Aro196: KDEC C3MCODBCO2 $\rightarrow .35$ MGLYOX + $.35 \mathrm{HO} 2+.35 \mathrm{CO}+.65 \mathrm{MMALANHY}+.65 \mathrm{HO} 2$ Aro198: KDEC C3MCODBCO2 $\rightarrow .35$ MGLYOX + $.35 \mathrm{HO} 2+.35 \mathrm{CO}+.65 \mathrm{MMALANHY}+.65 \mathrm{HO} 2$ Aro199: KDEC C3MCODBCO2 $\rightarrow .35$ MGLYOX + $.35 \mathrm{HO} 2+.35 \mathrm{CO}+.65$ MMALANHY + .65 HO2

Aro205: KDEC NTLFUO $\rightarrow$ ACCOMECHO + NO2 Aro206: KDEC NTLFUO $\rightarrow$ ACCOMECHO + NO2 Aro207: KDEC NTLFUO $\rightarrow$ ACCOMECHO + NO2 Aro218: KDEC C5DIALO $\rightarrow$ MALDIAL
Aro219: KDEC C5DIALO $\rightarrow$ MALDIAL

Aro220: KDEC C5DIALO $\rightarrow$ MALDIAL

Aro227: $\quad$ KDEC $\mathrm{C} 615 \mathrm{CO} 2 \mathrm{O} \rightarrow \mathrm{C} 5 \mathrm{DICARB}+\mathrm{CO}$

$+\mathrm{HO} 2$

Aro228: $\mathrm{KDEC}$ C615CO2O $\rightarrow$ C5DICARB $+\mathrm{CO}+$ $\mathrm{HO} 2$

Aro229: Only major channel taken

Aro290: KDEC PHENO $\rightarrow .71$ MALDALCO2H Aro237: $\quad$ KDEC NDNPHENO $\rightarrow$ NC4DCO2H $+\quad+.71$ GLYOX +.29 PBZQONE + HO2

$\mathrm{HNO} 3+\mathrm{CO}+\mathrm{CO}+\mathrm{NO} 2 \quad$ Aro291: $\quad$ KDEC PHENO $\rightarrow .71 \mathrm{MALDALCO} 2 \mathrm{H}+$ Aro238: $\quad$ KDEC NDNPHENO $\rightarrow$ NC4DCO2H +.71 GLYOX $+.29 \mathrm{PBZQONE}+\mathrm{HO} 2$

$\mathrm{HNO} 3+\mathrm{CO}+\mathrm{CO}+\mathrm{NO} 2$ $\mathrm{HNO} 3+\mathrm{CO}+\mathrm{CO}+\mathrm{NO} 2$

Aro241: KDEC CATECOOA $\rightarrow$ MALDALCO2H + Aro294: updated rco3_ooh+rco3_o3

$\mathrm{HCOCO} 2 \mathrm{H}+\mathrm{HO} 2+\mathrm{OH}$

Aro247: updated rco3_ooh+rco3_o3

Aro248: updated rco3_oh

Aro254: KDEC GLYOOA $\rightarrow .125 \mathrm{HCHO}+.18$

$\mathrm{GLYOO}+.82 \mathrm{HO} 2+.57 \mathrm{OH}+1.265 \mathrm{CO}$

Aro258: $\quad$ KDEC NCATECO $\rightarrow$ NC4DCO2H +

$\mathrm{HCOCO} 2 \mathrm{H}+\mathrm{HO} 2$

Aro259: $\quad$ KDEC NCATECO $\rightarrow$ NC4DCO2H +

$\mathrm{HCOCO} 2 \mathrm{H}+\mathrm{HO} 2$

Aro260: $\quad$ KDEC NCATECO $\rightarrow$ NC4DCO2H + $\mathrm{HCOCO} 2 \mathrm{H}+\mathrm{HO} 2$

Aro264: $\quad$ KDEC NPHENO $\rightarrow$ MALDALCO2H + GLYOX + NO2

Aro265: $\quad$ KDEC NPHENO $\rightarrow$ MALDALCO2H + GLYOX + NO2

Aro266: KDEC NPHENO $\rightarrow$ MALDALCO2H + GLYOX + NO2

Aro267: merged equations

Aro275: $\quad$ KDEC NNCATECO $\rightarrow$ NC4DCO2H +
$\mathrm{HCOCO} 2 \mathrm{H}+\mathrm{NO} 2$

Aro276: $\quad$ KDEC NNCATECO $\rightarrow$ NC4DCO2H + $\mathrm{HCOCO} 2 \mathrm{H}+\mathrm{NO} 2$

$\mathrm{HCOCO} 2 \mathrm{H}+\mathrm{NO} 2$

Aro292: KDEC PHENO $\rightarrow .71$ MALDALCO2H + .71 GLYOX + .29 PBZQONE + HO2 and Only major channel taken

Aro295: rco3_oh

Aro306: HOC6H4NO2 is a nitro-phenol

Aro312: $\mathrm{KDEC}$ PBZQO $\rightarrow \mathrm{C} 5 \mathrm{CO} 2 \mathrm{OHCO} 3$

Aro313: KDEC PBZQO $\rightarrow \mathrm{C} 5 \mathrm{CO} 2 \mathrm{OHCO} 3$

Aro314: KDEC PBZQO $\rightarrow$ C5CO2OHCO3 and Only major channel taken

Aro318: $\quad$ KDEC DNPHENO $\rightarrow$ NC4DCO2H + $\mathrm{HCOCO} 2 \mathrm{H}+\mathrm{NO} 2$

Aro319: $\quad$ KDEC DNPHENO $\rightarrow$ NC4DCO2H + $\mathrm{HCOCO} 2 \mathrm{H}+\mathrm{NO} 2$

Aro320: $\quad$ KDEC DNPHENO $\rightarrow$ NC4DCO2H + $\mathrm{HCOCO} 2 \mathrm{H}+\mathrm{NO} 2$

Aro325: KDEC BZEMUCO $\rightarrow .5$ EPXC4DIAL +.5 GLYOX $+.5 \mathrm{HO} 2+.5 \mathrm{C} 3 \mathrm{DIALO} 2+.5 \mathrm{C} 32 \mathrm{OH} 13 \mathrm{CO}$ Aro326: KDEC BZEMUCO $\rightarrow .5$ EPXC4DIAL +.5 GLYOX + .5 HO2 + .5 C3DIALO2 + .5 C32OH13CO Aro327: KDEC BZEMUCO $\rightarrow .5$ EPXC4DIAL +.5 GLYOX + .5 HO2 + .5 C3DIALO2 + .5 C32OH13CO 
and Only major channel taken

Aro335: KDEC NBZQO $\rightarrow$ C6CO4DB $+\mathrm{NO} 2$

Aro336: KDEC NBZQO $\rightarrow$ C6CO4DB $+\mathrm{NO} 2$

Aro337: KDEC NBZQO $\rightarrow$ C6CO4DB $+\mathrm{NO} 2$

Aro347: updated rco3_o3

Aro348: updated rco3_ooh

Aro349: updated rco3_oh

Aro353: Only major channel taken

Aro361: merged eq. under same rate c.

Aro365: KDEC BZBIPERO $\rightarrow$ GLYOX + HO2 +.5 BZFUONE +.5 BZFUONE

Aro366: KDEC BZBIPERO $\rightarrow$ GLYOX $+\mathrm{HO} 2+.5$ BZFUONE +.5 BZFUONE and Only major channel taken

Aro368: KROPRIM $^{*} \mathrm{O} 2$ fast reaction $\mathrm{C} 6 \mathrm{H} 5 \mathrm{CH} 2 \mathrm{O}=$ BENZAL + HO2

Aro370: KROPRIM*O2 fast reaction $\mathrm{C} 6 \mathrm{H} 5 \mathrm{CH} 2 \mathrm{O}$ $=$ BENZAL $+\mathrm{HO} 2$

Aro371: KROPRIM $^{*} \mathrm{O} 2$ fast reaction $\mathrm{C} 6 \mathrm{H} 5 \mathrm{CH} 2 \mathrm{O}=$ BENZAL + $\mathrm{HO} 2$ and $\mathrm{C} 6 \mathrm{H} 5 \mathrm{CH} 2 \mathrm{OH}$ replaced with its ox. product BENZAL

Aro372: merged eq under the same rate $\mathrm{C}$.

Aro373: merged eq under the same rate $\mathrm{C}$.

Aro375: $\quad$ KDEC TLBIPERO $\rightarrow .6$ GLYOX +.4

MGLYOX $+\mathrm{HO} 2+.2$ ZCODC23DBCOD +.2

C5DICARB +.2 TLFUONE +.2 BZFUONE +.2

MALDIAL

KDEC TLBIPERO $\rightarrow .6$ GLYOX +.4

MGLYOX $+\mathrm{HO} 2+.2$ ZCODC23DB COD +.2 C5DICARB +.2 TLFUONE +.2 BZFUONE +.2 MALDIAL

Aro378: Only major channel and KDEC TLBIPERO $\rightarrow .6$ GLYOX +.4 MGLYOX $+\mathrm{HO} 2+.2$ ZCODC $23 \mathrm{DB}$
COD +.2 C5DICARB +.2 TLFUONE +.2 BZ-

Aro419: branching ratios from Roth et al 2010

FUONE +.2 MALDIAL

Aro380: $\quad$ KDEC MGLOOB $\rightarrow .125 \mathrm{CH} 3 \mathrm{CHO}+$ $.695 \mathrm{CH} 3 \mathrm{CO} 3+.57 \mathrm{CO}+.57 \mathrm{OH}+.125 \mathrm{HO} 2+.18$

MGLOO

Aro381: merged equations

Aro388: KDEC CRESO $\rightarrow .68 \mathrm{C} 5 \mathrm{CO} 14 \mathrm{OH}+.68$

GLYOX + HO2 + .32 PTLQONE

Aro389: KDEC CRESO $\rightarrow .68 \mathrm{C} 5 \mathrm{CO} 14 \mathrm{OH}+.68$ GLYOX + HO2 + .32 PTLQONE

Aro390: KDEC CRESO $\rightarrow .68 \mathrm{C} 5 \mathrm{CO} 14 \mathrm{OH}+.68$

GLYOX + HO2 + .32 PTLQONE and Only major channel taken

Aro393:
+ NO2

Aro394: KDEC NCRESO $\rightarrow$ C5CO14OH + GLYOX

$+\mathrm{NO} 2$

Aro395: KDEC NCRESO $\rightarrow$ C5CO14OH + GLYOX

$+\mathrm{NO} 2$ and Only major channel taken

Aro396: TOL1OHNO2 is a nitro-phenol

Aro399: KDEC MCATECOOA $\rightarrow$ MC3ODBCO2H

$+\mathrm{HCOCO} 2 \mathrm{H}+\mathrm{HO} 2+\mathrm{OH}$

Aro404: updated rco3_oh

Aro405: updated rco3_o3

Aro406: updated rco3_ooh

Aro410: Only major channel taken

Aro415: KDEC TLEMUCO $\rightarrow .5$ C3DIALO $2+.5$

$\mathrm{CO} 2 \mathrm{H} 3 \mathrm{CHO}+.5 \mathrm{EPXC} 4 \mathrm{DIAL}+.5$ MGLYOX +.5 Aro495:

$\mathrm{HO} 2 \quad \mathrm{CH} 3 \mathrm{CO} 3$

Aro416: KDEC TLEMUCO $\rightarrow .5$ C3DIALO2 +.5 Aro496:

$\mathrm{CO} 2 \mathrm{H} 3 \mathrm{CHO}+.5 \mathrm{EPXC} 4 \mathrm{DIAL}+.5 \mathrm{MGLYOX}+.5 \mathrm{CH} 3 \mathrm{CO} 3$

$\mathrm{HO} 2$ and Only major channel taken

Aro417: branching ratios from Roth et al 2010

Aro497:

CH3CO3

Aro465: KDEC NPTLQO $\rightarrow$ C7CO4DB $+\mathrm{NO} 2$

KDEC NPTLQO $\rightarrow \mathrm{C} 7 \mathrm{CO} 4 \mathrm{DB}+\mathrm{NO} 2$

Aro470: KDEC PTLQO $\rightarrow$ C6CO2OHCO3

Aro471: KDEC PTLQO $\rightarrow$ C6CO2OHCO3

Aro472: Only major channel taken and KDEC

Aro482: $\quad$ KDEC MNNCATECO $\rightarrow$ NC4MDCO2H

$\mathrm{HCOCO} 2 \mathrm{H}+\mathrm{NO} 2$

Aro487: KDEC MNCATECO $\rightarrow$ NC4MDCO2H + $\mathrm{HCOCO} 2 \mathrm{H}+\mathrm{HO} 2$ $\mathrm{HCOCO} 2 \mathrm{H}+\mathrm{HO} 2$

$\mathrm{HCOCO} 2 \mathrm{H}+\mathrm{HO} 2$

Aro493: RO2 with ether function.

Aro418: branching ratios from Roth et al 2010
KDEC MCOCOMOXO $\rightarrow$ HCHO + KDEC MCOCOMOXO $\rightarrow$ HCHO + KDEC MCOCOMOXO $\rightarrow$ HCHO + 
Aro503: KDEC NDNCRESO $\rightarrow$ NC4MDCO2H + Aro540: we use DIET35TOL(from MCM) as repre$\mathrm{HNO} 3+\mathrm{CO}+\mathrm{CO}+\mathrm{NO} 2$

Aro504: $\quad$ KDEC NDNCRESO $\rightarrow$ NC4MDCO2H + Aro541: For consistency, we use the same products as $\mathrm{HNO} 3+\mathrm{CO}+\mathrm{CO}+\mathrm{NO} 2$

Aro505: $\quad$ KDEC NDNCRESO $\rightarrow$ NC4MDCO2H + Aro543: $\mathrm{KDEC} \mathrm{CH} 2 \mathrm{OOB} \rightarrow .24 \mathrm{CH} 2 \mathrm{OO}+.40 \mathrm{CO}+$ $\mathrm{HNO} 3+\mathrm{CO}+\mathrm{CO}+\mathrm{NO} 2 \quad .36 \mathrm{HO} 2+.36 \mathrm{CO}+.36 \mathrm{OH}$ and $\mathrm{H} 2 \mathrm{Osubs} \mathrm{PHCHOO}$ $\rightarrow .625 \mathrm{PHCOOH}+.375 \mathrm{BENZAL}+.375 \mathrm{H} 2 \mathrm{O} 2$

Aro508: $\quad$ KDEC DNCRESO $\rightarrow$ NC4MDCO2H + Aro546: KDEC NSTYRENEO $\rightarrow$ NO2 $+\mathrm{HCHO}+$ $\mathrm{HCOCO} 2 \mathrm{H}+\mathrm{NO} 2$

Aro509: $\quad$ KDEC DNCRESO $\rightarrow$ NC4MDCO2H + Aro547: KDEC NSTYRENEO $\rightarrow$ NO2 $+\mathrm{HCHO}+$ $\mathrm{HCOCO} 2 \mathrm{H}+\mathrm{NO} 2$

Aro510: $\quad$ KDEC DNCRESO $\rightarrow$ NC4MDCO2H + $\mathrm{HCOCO} 2 \mathrm{H}+\mathrm{NO} 2$

Aro517: updated rco3_oh

Aro518: updated rco3_ooh+rco3_o3

Aro528: For consistency, we use the same products as for TOLUENE

Aro529: For consistency, we use the same products as for TOLUENE

Aro530: For consistency, we use the same products as for TOLUENE

Aro531: For consistency, we use the same products as for TOLUENE

Aro532: For consistency, we use the same products as for TOLUENE

Aro533: For consistency, we use the same products as for TOLUENE

Aro534: For consistency, we use the same products as for TOLUENE

Aro535: For consistency, we use the same products as for TOLUENE

Aro536: For consistency, we use the same products as for TOLUENE

Aro537: For consistency, we use the same products as for TOLUENE

Aro538: merged under same rate constant

Aro539: For consistency, we use the same products as for TOLUENE
The following rate constants have been updated (respect to those used in MCM) Groß (2013) rco3_oh $=0.69$

rco3_o3 $=0.10$

rco3_ooh $=0.21$

- 2) Species which react fast are substituted with the respective products of the major loss pathway (see MIM2). This includes $\mathrm{ROO}+\mathrm{H} 2 \mathrm{O}$ reactions

- 3) Alcohol and the aldehyde channels are minor of an already minor channel for $\mathrm{RO} 2$. We remove them.

- 4) Channel of $\mathrm{OH}$ formation added in reactions with $\mathrm{KRO} 2 \mathrm{HO} 2$

- 5) Alkyl nitrates rate constant have been modified to be temperature dependent

- 6) LXYL and LTMB Rate constant for lumped aromatics were estimated following the procedure below:

- 1) Several rate constant have been defined at the beginning of the gas.eqn file for practical reasons (but rate constants themselves have not been modified) $\mathrm{KRO} 2 \mathrm{AP} \quad=1.00 \mathrm{E}-11$

KRO2sHORO2 $=1.40 \mathrm{E}-12$

KRO2tHORO2 $=1.60 \mathrm{E}-13$

$\mathrm{KRO} 2 \mathrm{pRO}=1.30 \mathrm{E}-12$

$\mathrm{KRO2SRO2}=2.50 \mathrm{E}-13$

$\mathrm{KRO2tRO2}=6.70 \mathrm{E}-15$

$\mathrm{KRO} 2 \mathrm{pORO2}=2.00 \mathrm{E}-12$

$\mathrm{KRO2}$ SORO2 $=8.80 \mathrm{E}-12$

KRO2tORO2 $=9.20 \mathrm{E}-14$

KPHENO2NO2 $=7.00 \mathrm{E}-12$

and rchohch2o2_oh $=0.10$ from Liu et al

(2013) and Groß (2013)
Rate constant estimation for lumped species:

\section{LXYL:}

1. LXYL + NO3

The rate constant is the average of the $-\mathrm{m},-\mathrm{p},-\mathrm{o}$ rate constants.

$\mathrm{Rc}=(4.10 \mathrm{E}-16+2.60 \mathrm{E}-16+5.00 \mathrm{E}-16) / 3=3.9 \mathrm{E}-16$

$2 .: \mathrm{XYL}+\mathrm{OH}$ For the reaction with $\mathrm{OH}$ a different approach is taken:

Rate constant is estimated by the average of the rate constant times the branching ratio.

A. for the xxxbipero channel

-O $1.36 \mathrm{E}-11 * 0.55=0.748 \mathrm{E}-11$

$-\mathrm{M} 2.31 \mathrm{E}-11 * 0.50=1.155 \mathrm{E}-11$

$-\mathrm{P} 1.43 \mathrm{E}-11 * 0.625=0.894 \mathrm{E}-11$ 


\section{$\operatorname{Rc}($ average $)=0.932 \mathrm{E}-11$}

B. for the xxxepoxuc channel -O $1.36 \mathrm{E}-11 * 0.24=0.326 \mathrm{E}-11$ -M 2.31E-11 *0.29 $=0.670 \mathrm{E}-11$ -P $1.43 \mathrm{E}-11 * 0.155=0.221 \mathrm{E}-11$ $\operatorname{Rc}($ ave $)=0.406 \mathrm{E}-11$

C. for the xxxylo2 channel -O $1.36 \mathrm{E}-11 * 0.05=0.068 \mathrm{E}-11$ -M 2.31E-11 *0.04 =0.092 E-11 -P 1.43E-11*0.10 = 0.143 E-11 $\mathrm{Rc}($ ave $)=0.101 \mathrm{E}-11$

D. for the xxxylol channel -O $1.36 \mathrm{E}-11 * 0.16=0.218 \mathrm{E}-11$ -M $2.31 \mathrm{E}-11 * 0.17=0.393 \mathrm{E}-11$
-P $1.43 \mathrm{E}-11 * 0.12=0.172 \mathrm{E}-11$ $\operatorname{Rc}($ ave $)=0.261 \mathrm{E}-11$

\section{LTMB:}

1. $\mathrm{LTMB}+\mathrm{NO} 3$

Analogous to LXYL

$(1.90+1.80+0.88) \mathrm{E}-15 / 3=1.52 \mathrm{E}-15$

2. $\mathrm{LTMB}+\mathrm{OH}$

A. for the xxxbipero

-O $3.27 \mathrm{E}-11 * 0.70=2.289 \mathrm{E}-11$

-M 3.25E-11*0.61 $=1.983 \mathrm{E}-11$

-P 5.67E-11*0.79 = $4.479 \mathrm{E}-11$

$\mathrm{Rc}($ ave $)=2.917 \mathrm{E}-11$

B. for the xxxepoxuc

-O 3.27E- $11 * 0.21=0.687 \mathrm{E}-11$
-M 3.25E-11*0.30=1.005 E-11

-P 5.67E-11 * $0.14=0.794 \mathrm{E}-11$

$\mathrm{Rc}($ ave $)=0.827 \mathrm{E}-11$

C. for the xxxylo2

-O 3.27E-11*0.06 = 0.196 E-11

-M 3.25E-11*0.06 $=0.201 \mathrm{E}-11$

-P 5.67E-11*0.03 = 0.170 E-11

$\operatorname{Rc}($ ave $)=0.189 \mathrm{E}-11$

D. for the xxxylol

-O 3.27E-11*0.03 = 0.098 E-11 -M 3.25E-11*0.03=0.100 E-11 -P 5.67E-11*0.04 = 0.226 E-11

$\operatorname{Rc}($ ave $)=0.141 \mathrm{E}-11$

- END of Aromatic gas phase reactions notes -

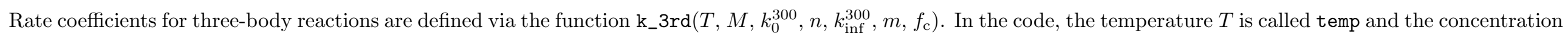
of "air molecules" $M$ is called cair. Using the auxiliary variables $k_{0}(T), k_{\text {inf }}(T)$, and $k_{\text {ratio }}$, k_3rd is defined as:

$$
\begin{aligned}
k_{0}(T) & =k_{0}^{300} \times\left(\frac{300 \mathrm{~K}}{T}\right)^{n} \\
k_{\text {inf }}(T) & =k_{\text {inf }}^{300} \times\left(\frac{300 \mathrm{~K}}{T}\right)^{m} \\
k_{\text {ratio }} & =\frac{k_{0}(T) M}{k_{\text {inf }}(T)} \\
k_{\text {_3rd }} & =\frac{k_{0}(T) M}{1+k_{\text {ratio }}} \times f_{\mathrm{c}}^{\left(\frac{1}{1+\left(\log _{10}\left(k_{\text {ratio }}\right)\right)^{2}}\right)}
\end{aligned}
$$


A similar function, called $\mathrm{k}_{-} 3 \mathrm{rd}_{-}$iupac here, is used by Atkinson et al. (2005) for three-body reactions. It has the same function parameters as $\mathrm{k}_{-} 3 \mathrm{rd}$ and it is defined as:

$$
\begin{aligned}
k_{0}(T) & =k_{0}^{300} \times\left(\frac{300 \mathrm{~K}}{T}\right)^{n} \\
k_{\text {inf }}(T) & =k_{\text {inf }}^{300} \times\left(\frac{300 \mathrm{~K}}{T}\right)^{m} \\
k_{\text {ratio }} & =\frac{k_{0}(T) M}{k_{\text {inf }}(T)} \\
N & =0.75-1.27 \times \log _{10}\left(f_{\mathrm{c}}\right) \\
\text { k_3rd_iupac }_{\text {_ratio }} & =\frac{k_{0}(T) M}{1+k_{\text {ratio }}} \times f_{\mathrm{c}}^{\left(\frac{1}{1+\left(\log _{10}\left(k_{\text {ratio }} / N\right)^{2}\right.}\right)}
\end{aligned}
$$


Table 2: Photolysis reactions. Labels follow same nomenclature as for table 1, with the addition of "J" meaning photolysis equations. Rate coefficients definition are taken from (Taraborrelli et al. 2009).

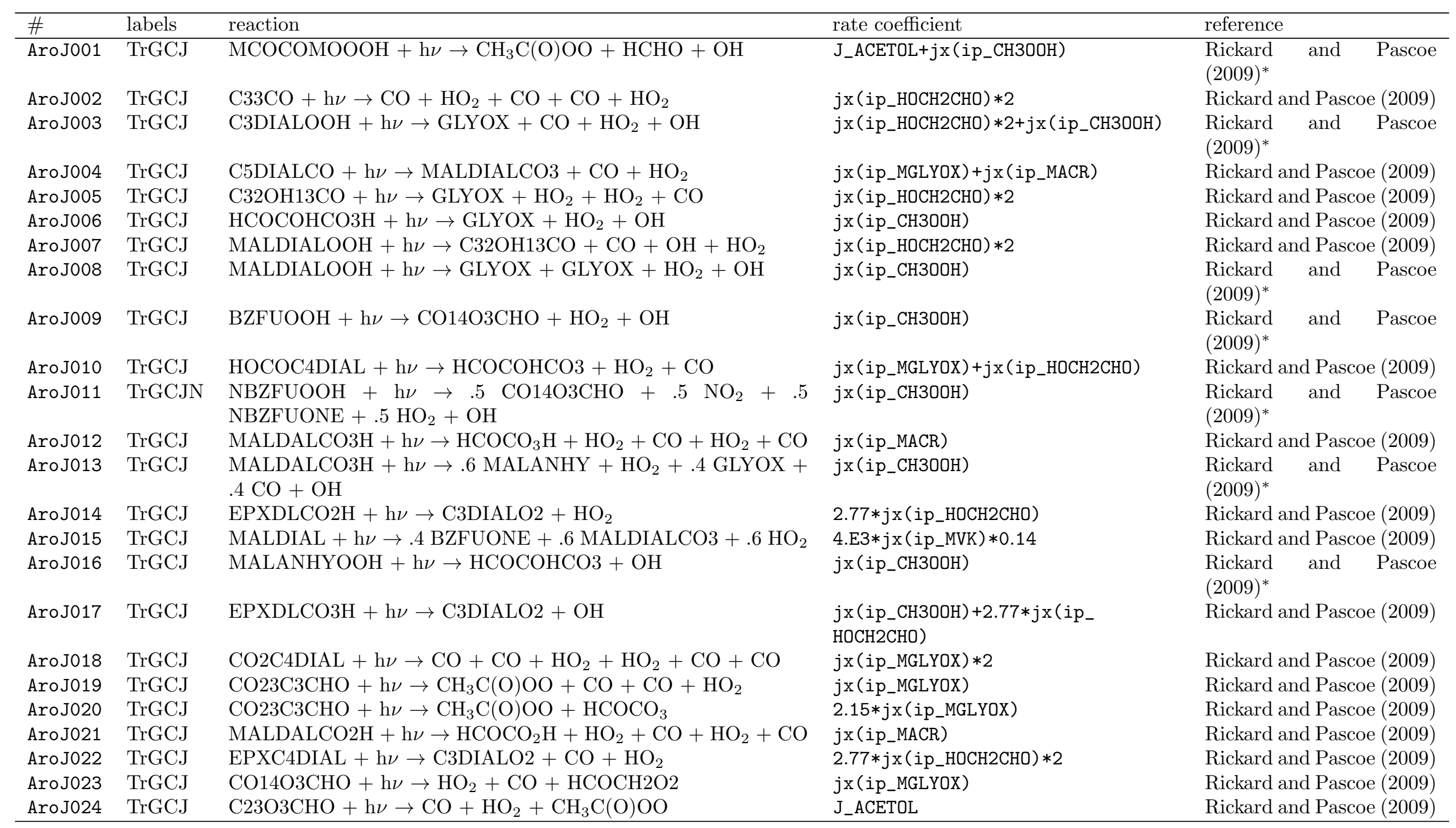


Table 2: Photolysis reactions (... continued)

\begin{tabular}{|c|c|c|c|c|}
\hline \# & labels & reaction & rate coefficient & reference \\
\hline AroJ025 & TrGCJ & $\mathrm{C} 54 \mathrm{CO}+\mathrm{h} \nu \rightarrow \mathrm{HO}_{2}+\mathrm{CO}+\mathrm{CO}+\mathrm{CO}+\mathrm{CH}_{3} \mathrm{C}(\mathrm{O}) \mathrm{OO}$ & $\begin{array}{l}\text { jx (ip_MGLYOX) }+2.15 * j x(\text { ip_MGLYOX }) \\
* 2\end{array}$ & Rickard and Pascoe (2009) \\
\hline AroJ026 & TrGCJ & $\mathrm{C} 5134 \mathrm{CO} 2 \mathrm{OH}+\mathrm{h} \nu \rightarrow \mathrm{CO} 23 \mathrm{C} 3 \mathrm{CHO}+\mathrm{HO}_{2}+\mathrm{CO}+\mathrm{HO}_{2}$ & $\begin{array}{l}\text { jx (ip_HOCH2CHO })+2.15 * j x\left(i p_{-}\right. \\
\text {MGLYOX) }\end{array}$ & Rickard and Pascoe (2009) \\
\hline AroJ027 & TrGCJ & $\mathrm{C} 5 \mathrm{DIALOOH}+\mathrm{h} \nu \rightarrow \mathrm{MALDIAL}+\mathrm{CO}+\mathrm{HO}_{2}+\mathrm{OH}$ & jx (ip_CH3OOH) +jx (ip_MACR) & $\begin{array}{lll}\text { Rickard } & \text { and } & \text { Pascoe } \\
(2009)^{*} & & \end{array}$ \\
\hline AroJ028 & TrGCNJ & $\mathrm{NPXYFUOOH}+\mathrm{h} \nu \rightarrow \mathrm{C} 23 \mathrm{O} 3 \mathrm{CCHO}+\mathrm{NO}_{2}+\mathrm{OH}$ & jx (ip_CH30OH) & $\begin{array}{l}\text { Rickard and Pascoe } \\
(2009)^{*}\end{array}$ \\
\hline AroJ029 & TrGCJ & $\mathrm{C} 23 \mathrm{O} 3 \mathrm{CCHO}+\mathrm{h} \nu \rightarrow \mathrm{CO}+\mathrm{MCOCOMOXO} 2+\mathrm{HO}_{2}$ & jx (ip_HOCH2CHO) & Rickard and Pascoe (2009) \\
\hline AroJ030 & TrGCJ & $\mathrm{PXYFUOOH}+\mathrm{h} \nu \rightarrow \mathrm{C} 23 \mathrm{O} 3 \mathrm{CCHO}+\mathrm{HO}_{2}+\mathrm{OH}$ & jx (ip_CH3OOH) & $\begin{array}{lll}\text { Rickard and Pascoe } \\
(2009)^{*}\end{array}$ \\
\hline AroJ031 & TrGCJ & $\mathrm{C} 5 \mathrm{CO} 14 \mathrm{OH}+\mathrm{h} \nu \rightarrow \mathrm{CH}_{3} \mathrm{C}(\mathrm{O}) \mathrm{OO}+\mathrm{HCOCO}_{2} \mathrm{H}+\mathrm{HO}_{2}+\mathrm{CO}$ & jx (ip_MVK) & Rickard and Pascoe (2009) \\
\hline AroJ032 & TrGCJ & $\mathrm{C} 5 \mathrm{DICARB}+\mathrm{h} \nu \rightarrow .6 \mathrm{C} 5 \mathrm{CO} 14 \mathrm{O} 2+.6 \mathrm{HO}_{2}+.4 \mathrm{TLFUONE}$ & $\mathrm{jx}\left(\mathrm{ip} \_\mathrm{NO} 2\right) * 0.2$ & $\begin{array}{lll}\text { Rickard and } & \text { Pascoe } \\
(2009)^{*} & \end{array}$ \\
\hline AroJ033 & TrGCJ & $\begin{array}{l}\mathrm{MC} 3 \mathrm{ODBCO} 2 \mathrm{H}+\mathrm{h} \nu \rightarrow \mathrm{CH} 3 \mathrm{COCO} 2 \mathrm{H}+\mathrm{HO}_{2}+\mathrm{CO}+\mathrm{HO}_{2}+ \\
\mathrm{CO}\end{array}$ & jx (ip_MACR) & Rickard and Pascoe (2009) \\
\hline AroJ034 & TrGCJ & $\mathrm{C} 4 \mathrm{CO} 2 \mathrm{DCO} 3 \mathrm{H}+\mathrm{h} \nu \rightarrow \mathrm{HO}_{2}+\mathrm{CO}+\mathrm{C} 33 \mathrm{CO}+\mathrm{OH}$ & jx (ip_CH3OOH) $+j x\left(i p_{-} M G L Y O X\right)$ & Rickard and Pascoe (2009) \\
\hline AroJ035 & TrGCJ & $\mathrm{ACCOMECHO}+\mathrm{h} \nu \rightarrow \mathrm{MECOACETO} 2+\mathrm{HO}_{2}+\mathrm{CO}$ & jx (ip_HOCH2CHO) & Rickard and Pascoe (2009) \\
\hline AroJ036 & TrGCJ & $\mathrm{MMALNHYOOH}+\mathrm{h} \nu \rightarrow \mathrm{CO} 2 \mathrm{H} 3 \mathrm{CO} 3+\mathrm{OH}$ & jx (ip_CH3OOH) & $\begin{array}{lll}\text { Rickard and Pascoe } \\
(2009)^{*}\end{array}$ \\
\hline AroJ037 & TrGCJ & $\mathrm{C} 5 \mathrm{DICAROOH}+\mathrm{h} \nu \rightarrow \mathrm{MGLYOX}+\mathrm{GLYOX}+\mathrm{HO}_{2}+\mathrm{OH}$ & $\begin{array}{l}\text { jx (ip_CH } 300 \mathrm{H})+j \mathrm{x}\left(\mathrm{ip} \_\mathrm{HOCH} 2 \mathrm{CHO}\right) \\
+\mathrm{J} \_\mathrm{ACETOL}\end{array}$ & $\frac{\text { Rickard and }}{(2009)^{*}}$ \\
\hline AroJ038 & TrGCNJ & $\mathrm{NTLFUOOH}+\mathrm{h} \nu \rightarrow \mathrm{ACCOMECHO}+\mathrm{NO}_{2}+\mathrm{OH}$ & jx(ip_CH3OOH) & $\begin{array}{l}\text { Rickard and } \\
(2009)^{*}\end{array}$ \\
\hline AroJ039 & TrGCJ & $\mathrm{MECOACEOOH}+\mathrm{h} \nu \rightarrow \mathrm{CH}_{3} \mathrm{C}(\mathrm{O}) \mathrm{OO}+\mathrm{HCHO}+\mathrm{OH}$ & jx (ip_CH3OOH) & $\begin{array}{ll}\text { Rickard and Pascoe } \\
(2009)^{*}\end{array}$ \\
\hline AroJ040 & TrGCJ & $\mathrm{C} 4 \mathrm{MALOHOOH}+\mathrm{h} \nu \rightarrow$ GLYOX + MGLYOX $+\mathrm{HO}_{2}+\mathrm{OH}$ & jx (ip_CH3OOH) & Rickard and Pascoe (2009) \\
\hline AroJ041 & TrGCJ & $\mathrm{C} 4 \mathrm{MALOHOOH}+\mathrm{h} \nu \rightarrow \mathrm{CO} 2 \mathrm{H} 3 \mathrm{CHO}+\mathrm{HO}_{2}+\mathrm{CO}+\mathrm{OH}$ & $2.77 *$ jx $($ ip_HOCH $2 \mathrm{CHO}) * 2$ & Rickard and Pascoe $(\overline{2009})$ \\
\hline AroJ042 & TrGCJ & $\begin{array}{l}\mathrm{C} 5 \mathrm{CO} 14 \mathrm{OOH}+\mathrm{h} \nu \rightarrow .83 \text { MALANHY }+.83 \mathrm{CH}_{3} \mathrm{O}_{2}+.17 \\
\text { MGLYOX }+.17 \mathrm{HO}_{2}+.17 \mathrm{CO}+\mathrm{OH}\end{array}$ & jx (ip_CH3OOH) & $\begin{array}{lll}\text { Rickard } & \text { and } & \text { Pascoe } \\
(2009)^{*} & \end{array}$ \\
\hline AroJ043 & TrGCJ & $\begin{array}{l}\mathrm{C} 4 \mathrm{CODBCO} 3 \mathrm{H}+\mathrm{h} \nu \rightarrow .35 \mathrm{MGLYOX}+.35 \mathrm{HO}_{2}+.35 \mathrm{CO}+ \\
.65 \text { MMALANHY }+.65 \mathrm{HO}_{2}+\mathrm{OH}\end{array}$ & $\mathrm{jx}\left(\mathrm{ip} \_\mathrm{CH} 3 \mathrm{OOH}\right)$ & $\begin{array}{ll}\text { Rickard and Pascoe } \\
(2009)^{*}\end{array}$ \\
\hline AroJ044 & TrGCJ & $\begin{array}{l}\mathrm{C} 4 \mathrm{CODBCO} 3 \mathrm{H}+\mathrm{h} \nu \rightarrow \mathrm{HCOCO}_{3} \mathrm{H}+\mathrm{HO}_{2}+\mathrm{CO}+ \\
\mathrm{CH}_{3} \mathrm{C}(\mathrm{O}) \mathrm{OO}\end{array}$ & jx (ip_MACR) & Rickard and Pascoe (2009) \\
\hline
\end{tabular}


Table 2: Photolysis reactions (... continued)

\begin{tabular}{|c|c|c|c|c|}
\hline \# & labels & reaction & rate coefficient & reference \\
\hline AroJ045 & TrGCJ & $\mathrm{TLFUOOH}+\mathrm{h} \nu \rightarrow \mathrm{ACCOMECHO}+\mathrm{HO}_{2}+\mathrm{OH}$ & jx (ip_CH3OOH) & $\begin{array}{lll}\text { Rickard and Pascoe } \\
(2009)^{*}\end{array}$ \\
\hline AroJ046 & TrGCJ & $\mathrm{C} 23 \mathrm{O} 3 \mathrm{CCO} 3 \mathrm{H}+\mathrm{h} \nu \rightarrow \mathrm{OH}+\mathrm{MCOCOMOXO} 2$ & $\mathrm{jx}\left(\mathrm{ip} \_\mathrm{CH} 3 \mathrm{OOH}\right)$ & Rickard and Pascoe (2009) \\
\hline AroJ047 & TrGCJ & $\begin{array}{l}\mathrm{MC} 3 \mathrm{ODBCO} 3 \mathrm{H}+\mathrm{h} \nu \rightarrow \mathrm{CH} 3 \mathrm{COCO} 3 \mathrm{H}+\mathrm{HO}_{2}+\mathrm{CO}+\mathrm{HO}_{2}+ \\
\mathrm{CO}\end{array}$ & jx (ip_MACR) & Rickard and Pascoe $(\overline{2009})$ \\
\hline AroJ048 & TrGCJ & $\begin{array}{l}\text { MC3ODBCO3H }+\mathrm{h} \nu \rightarrow .35 \text { GLYOX }+.35 \mathrm{CH}_{3} \mathrm{O}_{2}+.35 \mathrm{CO}+ \\
.65 \text { MMALANHY }+.65 \mathrm{HO}_{2}+\mathrm{OH}\end{array}$ & jx (ip_CH3OOH) & $\frac{\text { Rickard and Pascoe }}{(2009)^{*}}$ \\
\hline AroJ049 & TrGCJ & $\mathrm{ACCOMECO} 3 \mathrm{H}+\mathrm{h} \nu \rightarrow \mathrm{MECOACETO} 2+\mathrm{OH}$ & jx (ip_CH3OOH) & Rickard and Pascoe (2009) \\
\hline AroJ050 & TrGCJ & $\begin{array}{l}\text { PHENOOH }+\mathrm{h} \nu \rightarrow .71 \text { MALDALCO2H }+.71 \text { GLYOX }+.29 \\
\text { PBZQONE }+\mathrm{HO}_{2}+\mathrm{OH}\end{array}$ & $\mathrm{jx}\left(\mathrm{ip} \mathrm{CH}_{-} \mathrm{OOOH}\right)$ & $\begin{array}{l}\text { Rickard and Pascoe } \\
(2009)^{*}\end{array}$ \\
\hline AroJ051 & TrGCJ & $\mathrm{C} 6 \mathrm{CO} 4 \mathrm{DB}+\mathrm{h} \nu \rightarrow \mathrm{C} 4 \mathrm{CO} 2 \mathrm{DBCO} 3+\mathrm{HO}_{2}+\mathrm{CO}$ & jx (ip_MGLYOX) $* 2$ & Rickard and Pascoe (2009) \\
\hline AroJ052 & TrGCJ & $\mathrm{C} 5 \mathrm{CO} 2 \mathrm{DCO} 3 \mathrm{H}+\mathrm{h} \nu \rightarrow \mathrm{CH}_{3} \mathrm{C}(\mathrm{O}) \mathrm{OO}+\mathrm{C} 33 \mathrm{CO}+\mathrm{OH}$ & $\mathrm{jx}\left(\mathrm{ip} \_\mathrm{CH} 30 \mathrm{OH}\right)+\mathrm{jx}\left(\mathrm{ip} \_\right.$MGLYOX $)$ & Rickard and Pascoe (2009) \\
\hline AroJ053 & TrGCNJ & $\begin{array}{l}\mathrm{NDNPHENOOH}+\mathrm{h} \nu \rightarrow \mathrm{NC} 4 \mathrm{DCO} 2 \mathrm{H}+\mathrm{HNO}_{3}+\mathrm{CO}+\mathrm{CO}+ \\
\mathrm{NO}_{2}+\mathrm{OH}\end{array}$ & jx (ip_CH3OOH) & $\begin{array}{l}\text { Rickard and } \\
(2009)^{*}\end{array}$ \\
\hline AroJ054 & TrGCNJ & $\begin{array}{l}\text { BZBIPERNO3 }+\mathrm{h} \nu \rightarrow \text { GLYOX }+\mathrm{HO}_{2}+.5 \text { BZFUONE }+.5 \\
\text { BZFUONE }+\mathrm{NO}_{2}\end{array}$ & J_IC3H7NO3 & $\begin{array}{l}\text { Rickard and } \\
(2009)^{*}\end{array}$ \\
\hline AroJ055 & TrGCNJ & $\begin{array}{l}\text { HOC6H4NO2 }+\mathrm{h} \nu \\
\text { CPENTDIENKETENE }\end{array}$ & jx (ip_HOC6H4NO2) & see note \\
\hline AroJ056 & TrGCJ & $\begin{array}{l}\text { CPENTDIENKETENE }+\mathrm{h} \nu \rightarrow \mathrm{CO}_{2}+\mathrm{CO}+2 \mathrm{HO}_{2}+ \\
\text { MALDIAL }\end{array}$ & J_KETENE & see note \\
\hline AroJ057 & TrGCJ & $\mathrm{C} 5 \mathrm{COOHCO} 3 \mathrm{H}+\mathrm{h} \nu \rightarrow \mathrm{HOCOC} 4 \mathrm{DIAL}+\mathrm{HO}_{2}+\mathrm{CO}+\mathrm{OH}$ & jx (ip_CH3OOH) & Rickard and Pascoe 2009) \\
\hline AroJ058 & TrGCJ & $\begin{array}{l}\text { BZEPOXMUC }+\mathrm{h} \nu \rightarrow .5 \text { C5DIALO2 }+1.5 \mathrm{HO}_{2}+1.5 \mathrm{CO}+.5 \\
\text { MALDIAL }\end{array}$ & $\mathrm{jx}\left(\mathrm{ip} \_\mathrm{NO2}\right) * 0.1$ & Rickard and Pascoe (2009) \\
\hline AroJ059 & TrGCJN & $\mathrm{NPHEN} 1 \mathrm{OOH}+\mathrm{h} \nu \rightarrow \mathrm{NPHEN} 1 \mathrm{O}+\mathrm{OH}$ & $\mathrm{jx}\left(\mathrm{ip} \_\mathrm{CH} 30 \mathrm{OH}\right)$ & Rickard and Pascoe 2009) \\
\hline AroJ060 & TrGCJ & BZEMUCCO $+\mathrm{h} \nu \rightarrow$ HCOCOHCO3 + C3DIALO 2 & jx (ip_HOCH2CHO $) * 2+J_{-}$ACETOL & Rickard and Pascoe (2009) \\
\hline AroJ061 & TrGCJ & $\mathrm{BZEMUCCO} 2 \mathrm{H}+\mathrm{h} \nu \rightarrow \mathrm{C} 5 \mathrm{DIALO} 2+\mathrm{HO}_{2}$ & jx (ip_MACR) & Rickard and Pascoe (2009) \\
\hline AroJ062 & TrGCNJ & $\begin{array}{l}\mathrm{NNCATECOOH}+\mathrm{h} \nu \rightarrow \mathrm{NC} 4 \mathrm{DCO} 2 \mathrm{H}+\mathrm{HCOCO}_{2} \mathrm{H}+\mathrm{NO}_{2}+ \\
\mathrm{OH}\end{array}$ & $\mathrm{jx}\left(\mathrm{ip} \_\mathrm{CH} 30 \mathrm{OH}\right)$ & $\begin{array}{ll}\text { Rickard and } \\
(2009)^{*}\end{array}$ \\
\hline AroJ063 & TrGCJ & $\mathrm{C} 615 \mathrm{CO} 2 \mathrm{OOH}+\mathrm{h} \nu \rightarrow \mathrm{C} 5 \mathrm{DICARB}+\mathrm{CO}+\mathrm{HO}_{2}+\mathrm{OH}$ & $j x\left(i p \_M V K\right)+j x\left(i p_{C} C H 3 O O H\right)$ & Rickard and Pascoe (2009) \\
\hline AroJ064 & TrGCNJ & $\mathrm{NPHENOOH}+\mathrm{h} \nu \rightarrow$ MALDALCO2H + GLYOX $+\mathrm{OH}+\mathrm{NO}_{2}$ & J_IC3H7NO3 + jx (ip_CH3OOH) & Rickard and Pascoe $(\overline{2009})$ \\
\hline AroJ065 & TrGCNJ & $\mathrm{NCATECOOH}+\mathrm{h} \nu \rightarrow \mathrm{NC} 4 \mathrm{DCO} 2 \mathrm{H}+\mathrm{HCOCO}_{2} \mathrm{H}+\mathrm{HO}_{2}+\mathrm{OH}$ & jx (ip_CH3OOH) & $\begin{array}{ll}\text { Rickard and } \\
(2009)^{*}\end{array}$ \\
\hline AroJ066 & TrGCJ & $\mathrm{PBZQOOH}+\mathrm{h} \nu \rightarrow \mathrm{C} 5 \mathrm{CO} 2 \mathrm{OHCO} 3+\mathrm{OH}$ & jx (ip_CH3OOH) & Rickard and \\
\hline AroJ067 & TrGCJ & $\mathrm{BZOBIPEROH}+\mathrm{h} \nu \rightarrow$ MALDIALCO $3+\mathrm{GLYOX}+\mathrm{HO}_{2}$ & J_ACETOL & Rickard and Pascoe (2009) \\
\hline
\end{tabular}


Table 2: Photolysis reactions (... continued)

\begin{tabular}{|c|c|c|c|c|}
\hline \# & labels & reaction & rate coefficient & reference \\
\hline AroJ068 & TrGCJ & $\begin{array}{l}\text { BZBIPEROOH }+\mathrm{h} \nu \rightarrow \text { GLYOX }+\mathrm{HO}_{2}+.5 \text { BZFUONE }+.5 \\
\text { BZFUONE }+\mathrm{OH}\end{array}$ & jx(ip_CH3OOH) & $\begin{array}{lll}\text { Rickard and } & \text { Pascoe } \\
(2009)^{*} & & \\
\end{array}$ \\
\hline AroJ069 & TrGCNJ & $\mathrm{NBZQOOH}+\mathrm{h} \nu \rightarrow \mathrm{C} 6 \mathrm{CO} 4 \mathrm{DB}+\mathrm{NO}_{2}+\mathrm{OH}$ & jx (ip_CH3OOH) & $\frac{\text { Rickard and Pascoe }}{(2009)^{*}}$ \\
\hline AroJ070 & TrGCJ & $\mathrm{CATEC} 1 \mathrm{OOH}+\mathrm{h} \nu \rightarrow \mathrm{CATEC} 1 \mathrm{O}+\mathrm{OH}$ & jx (ip_CH3OOH) & Rickard and Pascoe (2009) \\
\hline AroJ071 & TrGCJ & $\mathrm{C} 6125 \mathrm{CO}+\mathrm{h} \nu \rightarrow \mathrm{C} 5 \mathrm{CO} 14 \mathrm{O} 2+\mathrm{CO}+\mathrm{HO}_{2}$ & $j x\left(i p \_M G L Y O X\right)+j x\left(i p \_M V K\right)$ & Rickard and Pascoe (2009) \\
\hline AroJ072 & TrGCNJ & DNPHENOOH $+\mathrm{h} \nu \rightarrow \mathrm{NC} 4 \mathrm{DCO} 2 \mathrm{H}+\mathrm{HCOCO}_{2} \mathrm{H}+\mathrm{NO}_{2}+\mathrm{OH}$ & jx (ip_CH3OOH) & $\begin{array}{l}\text { Rickard and Pascoe } \\
(2009)^{*}\end{array}$ \\
\hline AroJ073 & TrGCJ & $\mathrm{BZEMUCCO} 3 \mathrm{H}+\mathrm{h} \nu \rightarrow \mathrm{C} 5 \mathrm{DIALO} 2+\mathrm{OH}$ & $j x\left(i p \_C H 3 O O H\right)+j x\left(i p \_M A C R\right)$ & Rickard and Pascoe (2009) \\
\hline AroJ074 & TrGCJ & $\mathrm{C} 6 \mathrm{H} 5 \mathrm{OOH}+\mathrm{h} \nu \rightarrow \mathrm{C} 6 \mathrm{H} 5 \mathrm{O}+\mathrm{OH}$ & jx (ip_CH3OOH) & 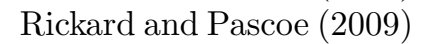 \\
\hline AroJ075 & TrGCJ & $\begin{array}{l}\text { BZEMUCOOH }+\mathrm{h} \nu \rightarrow .5 \mathrm{EPXC} 4 \mathrm{DIAL}+.5 \mathrm{GLYOX}+.5 \mathrm{HO}_{2} \\
+.5 \mathrm{C} 3 \mathrm{DIALO} 2+.5 \mathrm{C} 32 \mathrm{OH} 13 \mathrm{CO}+\mathrm{OH}\end{array}$ & $\begin{array}{l}\text { jx }(\text { ip_CH} 3 \mathrm{OOH})+j \times\left(i p_{-} \mathrm{HOCH} 2 \mathrm{CHO}\right) \\
* 2\end{array}$ & $\begin{array}{l}\text { Rickard and Pascoe } \\
(2009)^{*}\end{array}$ \\
\hline AroJ076 & TrGCJ & $\begin{array}{l}\text { BZEMUCOH }+\mathrm{h} \nu \rightarrow .5 \mathrm{EPXC} 4 \mathrm{DIAL}+.5 \mathrm{GLYOX}+.5 \mathrm{HO}_{2}+ \\
.5 \mathrm{C} 3 \mathrm{DIALO} 2+.5 \mathrm{C} 32 \mathrm{OH} 13 \mathrm{CO}+\mathrm{HO}_{2}\end{array}$ & jx (ip_HOCH2CHO) $* 2$ & Rickard and Pascoe \\
\hline AroJ077 & TrGCJN & BZEMUCNO $3+\mathrm{h} \nu \rightarrow$ EPXC4DIAL $+\mathrm{NO}_{2}+\mathrm{GLYOX}+\mathrm{HO}_{2}$ & $2.77 * \mathrm{jx}$ (ip_HOCH2CHO) & Rickard and Pascoe (2009) \\
\hline AroJ078 & TrGCJ & $\begin{array}{l}\text { TLEPOXMUC }+\mathrm{h} \nu \rightarrow .5 \mathrm{C} 615 \mathrm{CO} 2 \mathrm{O} 2+\mathrm{HO}_{2}+\mathrm{CO}+.5 \\
\text { EPXC4DIAL }+.5 \mathrm{CH}_{3} \mathrm{C}(\mathrm{O}) \mathrm{OO}\end{array}$ & $\mathrm{jx}\left(\mathrm{ip} \_\mathrm{NO} 2\right) * 0.1$ & Rickard and Pascoe 2009 \\
\hline AroJ079 & TrGCJ & $\mathrm{C} 6 \mathrm{H} 5 \mathrm{CH} 2 \mathrm{OOH}+\mathrm{h} \nu \rightarrow \mathrm{BENZAL}+\mathrm{HO}_{2}+\mathrm{OH}$ & jx (ip_CH3OOH) & $\begin{array}{lll}\text { Rickard and Pascoe } \\
(2009)^{*}\end{array}$ \\
\hline AroJ080 & TrGCJ & $\mathrm{C} 6 \mathrm{H} 5 \mathrm{CH} 2 \mathrm{NO} 3+\mathrm{h} \nu \rightarrow \mathrm{BENZAL}+\mathrm{HO}_{2}+\mathrm{NO}_{2}$ & $0.59 * J_{-}$IC3H7N03 & $\begin{array}{lll}\text { Rickard and Pascoe } \\
(2009)^{*}\end{array}$ \\
\hline AroJ081 & TrGCJ & $\mathrm{BENZAL}+\mathrm{h} \nu \rightarrow \mathrm{HO}_{2}+\mathrm{CO}+\mathrm{C} 6 \mathrm{H} 5 \mathrm{O} 2$ & jx (ip_BENZAL) & Atkinson et al. (2012) \\
\hline AroJ082 & TrGCJ & $\begin{array}{l}\text { TLBIPEROOH }+\mathrm{h} \nu \rightarrow .6 \text { GLYOX }+.4 \text { MGLYOX }+\mathrm{HO}_{2}+.2 \\
\text { C4MDIAL }+.2 \text { C5DICARB }+.2 \text { TLFUONE }+.2 \text { BZFUONE }+ \\
.2 \text { MALDIAL }+ \text { OH }\end{array}$ & jx(ip_CH3OOH) & $\frac{\text { Rickard and }}{(2009)^{*}}$ \\
\hline AroJ083 & TrGCJN & $\begin{array}{l}\text { TLBIPERNO } 3+\mathrm{h} \nu \rightarrow .6 \mathrm{GLYOX}+.4 \mathrm{MGLYOX}+\mathrm{HO}_{2}+.2 \\
\text { C4MDIAL }+.2 \mathrm{C} 5 \mathrm{DICARB}+.2 \mathrm{TLFUONE}+.2 \mathrm{BZFUONE}+ \\
.2 \text { MALDIAL }+\mathrm{NO}_{2}\end{array}$ & J_IC3H7NO3 & $\frac{\text { Rickard and }}{(2009)^{*}}$ \\
\hline AroJ084 & TrGCJ & $\mathrm{TLOBIPEROH}+\mathrm{h} \nu \rightarrow \mathrm{C} 5 \mathrm{CO} 14 \mathrm{O} 2+\mathrm{GLYOX}+\mathrm{HO}_{2}$ & J_ACETOL & Rickard and Pascoe (2009) \\
\hline AroJ085 & TrGCJ & $\begin{array}{l}\mathrm{CRESOOH}+\mathrm{h} \nu \rightarrow .68 \mathrm{C} 5 \mathrm{CO} 14 \mathrm{OH}+.68 \mathrm{GLYOX}+\mathrm{HO}_{2}+.32 \\
\text { PTLQONE }+\mathrm{OH}\end{array}$ & jx (ip_CH3OOH) & $\frac{\text { Rickard }}{(2009)^{*}}$ \\
\hline AroJ086 & TrGCNJ & $\begin{array}{l}\text { NCRESOOH }+\mathrm{h} \nu \rightarrow .68 \mathrm{C} 5 \mathrm{CO} 14 \mathrm{OH}+.68 \mathrm{GLYOX}+\mathrm{HO}_{2}+ \\
.32 \text { PTLQONE }+\mathrm{OH}+\mathrm{NO}_{2}\end{array}$ & J_IC3H7NO3 & $\frac{\text { Rickard }}{(2009)^{*}}$ \\
\hline AroJ087 & TrGCNJ & $\mathrm{NCRESOOH}+\mathrm{h} \nu \rightarrow \mathrm{C} 5 \mathrm{CO} 14 \mathrm{OH}+\mathrm{GLYOX}+\mathrm{NO}_{2}+\mathrm{OH}$ & jx (ip_CH3OOH) & $\frac{\text { Rickard }}{(2009)^{*}}$ \\
\hline
\end{tabular}


Table 2: Photolysis reactions (... continued)

\begin{tabular}{|c|c|c|c|c|}
\hline \# & labels & reaction & rate coefficient & reference \\
\hline AroJ088 & TrGCNJ & $\begin{array}{l}\text { TOL1OHNO2 }+\mathrm{h} \nu \\
\text { MCPENTDIENKETENE }\end{array} \rightarrow$ HONO + PHONOnitrop + & jx (ip_TOL1OHNO2) & see note \\
\hline AroJ089 & TrGCJ & $\begin{array}{l}\mathrm{C} 4 \mathrm{MDIAL}+\mathrm{h} \nu \rightarrow .3 \mathrm{C}_{3 \mathrm{MCODBCO}}+.3 \mathrm{HO}_{2}+.3 \mathrm{CO}+.3 \\
\text { MC3CODBCO } 3+.3 \mathrm{HO}_{2}+.3 \mathrm{CO}+.4 \text { PXYFUONE }\end{array}$ & 4.E3*jx (ip_MVK) $* 0.2$ & Rickard and Pascoe (2009) \\
\hline AroJ090 & TrGCJ & TLEMUCCO2H $+\mathrm{h} \nu \rightarrow \mathrm{C} 615 \mathrm{CO} 2 \mathrm{O} 2+\mathrm{HO}_{2}$ & jx (ip_MACR) & Rickard and Pascoe (2009) \\
\hline AroJ091 & TrGCJ & TLEMUCCO3H $+\mathrm{h} \nu \rightarrow \mathrm{C} 615 \mathrm{CO} 2 \mathrm{O} 2+\mathrm{OH}$ & $j \times\left(i p_{-} \mathrm{CH} 30 \mathrm{OH}\right)+j \times\left(i p_{-} \mathrm{MACR}\right)$ & Rickard and Pascoe $(\overline{2009})$ \\
\hline AroJ092 & TrGCJ & $\begin{array}{l}\text { TLEMUCOOH }+\mathrm{h} \nu \rightarrow .5 \text { C3DIALO } 2+.5 \mathrm{CO} 2 \mathrm{H} 3 \mathrm{CHO}+.5 \\
\text { EPXC4DIAL }+.5 \mathrm{MGLYOX}+.5 \mathrm{HO}_{2}+\mathrm{OH}\end{array}$ & $\begin{array}{l}\text { jx (ip_CH3OOH) }+2.77 * j \times\left(\text { ip }_{-}\right. \\
\text {HOCH } 2 \text { CHO })+J_{-} \text {ACETOL }\end{array}$ & Rickard and Pascoe \\
\hline AroJ093 & TrGCNJ & $\begin{array}{l}\text { TLEMUCNO3 }+\mathrm{h} \nu \rightarrow \text { EPXC4DIAL }+\mathrm{NO}_{2}+\mathrm{CH}_{3} \mathrm{C}(\mathrm{O}) \mathrm{OO}+ \\
\mathrm{CO}+\mathrm{HO}_{2}\end{array}$ & $\begin{array}{l}2.77 * j \times(\text { ip_HOCH} 2 \mathrm{CHO})+\mathrm{J}_{-} \\
\text {ACETOL }\end{array}$ & Rickard and Pascoe (2009) \\
\hline AroJ094 & TrGCJ & $\begin{array}{l}\text { TLEMUCCO }+\mathrm{h} \nu \rightarrow \mathrm{CH}_{3} \mathrm{C}(\mathrm{O}) \mathrm{OO}+\text { EPXC4DIAL }+\mathrm{CO}+ \\
\mathrm{HO}_{2}\end{array}$ & $\begin{array}{l}2.77 * j x\left(i p_{-} H_{O C H} 2 \mathrm{CHO}\right)+2.15 * j \times\left(i p_{-}\right. \\
\text {MGLYOX })\end{array}$ & Rickard and Pascoe (2009) \\
\hline AroJ095 & TrGCJ & $\mathrm{C} 6 \mathrm{H} 5 \mathrm{CO} 3 \mathrm{H}+\mathrm{h} \nu \rightarrow \mathrm{C} 6 \mathrm{H} 5 \mathrm{O} 2+\mathrm{OH}$ & jx (ip_CH3OOH) & Rickard and Pascoe 2009) \\
\hline AroJ096 & TrGCJ & $\mathrm{OXYL1OOH}+\mathrm{h} \nu \rightarrow \mathrm{TOL} 1 \mathrm{O}+\mathrm{OH}$ & jx (ip_CH3OOH) & Rickard and Pascoe (2009) \\
\hline AroJ097 & TrGCNJ & $\begin{array}{l}\text { MNCATECH }+\mathrm{h} \nu \\
\text { MCPENTDIENKETENE }\end{array}$ & jx(ip_TOL1OHNO2) & see note \\
\hline AroJ098 & TrGCJ & $\begin{array}{l}\text { MCPENTDIENKETENE }+\mathrm{h} \nu \rightarrow \mathrm{CO}_{2}+\mathrm{CO}+2 \mathrm{HO}_{2}+ \\
\text { C4MDIAL }\end{array}$ & J_KETENE & see note \\
\hline AroJ099 & TrGCNJ & $\begin{array}{l}\text { DNCRES }+\mathrm{h} \nu \rightarrow \underset{H O N O}{\rightarrow}+\underset{\mathrm{PHONOnitrop}}{+}+ \\
\text { MNCPENTDIENKETENE }+ \text { LhvDNCRES }+\mathrm{NO}_{2}\end{array}$ & jx (ip_TOL10HNO2) & see note \\
\hline AroJ100 & TrGCNJ & $\begin{array}{l}\text { MNCPENTDIENKETENE }+\mathrm{h} \nu \rightarrow \mathrm{CO}_{2}+\mathrm{CO}+2 \mathrm{HO}_{2}+ \\
\text { NC4MDCO2HN }\end{array}$ & J_KETENE & see note \\
\hline AroJ101 & TrGCNJ & DNPHEN $+\mathrm{h} \nu \rightarrow$ HONO + MNCPENTDIENKETENE $+\mathrm{NO}_{2}$ & jx(ip_HOC6H4NO2) & see note \\
\hline AroJ102 & TrGCJ & $\mathrm{MCATEC} 1 \mathrm{OOH}+\mathrm{h} \nu \rightarrow \mathrm{MCATEC} 1 \mathrm{O}+\mathrm{OH}$ & jx (ip_CH3OOH) & Rickard and Pascoe (2009) \\
\hline AroJ103 & TrGCNJ & $\mathrm{NPTLQOOH}+\mathrm{h} \nu \rightarrow \mathrm{C} 7 \mathrm{CO} 4 \mathrm{DB}+\mathrm{NO}_{2}+\mathrm{OH}$ & jx (ip_CH30OH) & $\begin{array}{lll}\text { Rickard and } & \text { Pascoe } \\
(2009)^{*} & & \\
\end{array}$ \\
\hline AroJ104 & TrGCJ & $\mathrm{PTLQOOH}+\mathrm{h} \nu \rightarrow \mathrm{C} 6 \mathrm{CO} 2 \mathrm{OHCO} 3+\mathrm{OH}$ & jx (ip_CH3OOH) & $\begin{array}{ll}\text { Rickard and Pascoe } \\
(2009)^{*}\end{array}$ \\
\hline AroJ105 & TrGCNJ & $\mathrm{NCRES} 1 \mathrm{OOH}+\mathrm{h} \nu \rightarrow \mathrm{NCRES} 1 \mathrm{O}+\mathrm{OH}$ & jx (ip_CH3OOH) & Rickard and Pascoe (2009) \\
\hline AroJ106 & TrGCNJ & $\begin{array}{l}\mathrm{MNNCATCOOH}+\mathrm{h} \nu \rightarrow \mathrm{NC} 4 \mathrm{MDCO} 2 \mathrm{HN}+\mathrm{HCOCO}_{2} \mathrm{H}+\mathrm{NO}_{2} \\
+\mathrm{OH}\end{array}$ & jx (ip_CH3OOH) & $\begin{array}{lll}\text { Rickard and Pascoe } \\
(2009)^{*}\end{array}$ \\
\hline AroJ107 & TrGCNJ & $\begin{array}{l}\mathrm{MNCATECOOH}+\mathrm{h} \nu \rightarrow \mathrm{NC} 4 \mathrm{MDCO} 2 \mathrm{HN}+\mathrm{HCOCO}_{2} \mathrm{H}+\mathrm{HO}_{2} \\
+\mathrm{OH}\end{array}$ & jx (ip_CH3OOH) & $\begin{array}{l}\text { Rickard and } \\
(2009)^{*}\end{array}$ \\
\hline AroJ108 & TrGCJ & $\mathrm{C} 7 \mathrm{CO} 4 \mathrm{DB}+\mathrm{h} \nu \rightarrow \mathrm{C} 5 \mathrm{CO} 2 \mathrm{DBCO} 3+\mathrm{HO}_{2}+\mathrm{CO}$ & jx (ip_MGLYOX) $* 2$ & Rickard and Pascoe (2009) \\
\hline
\end{tabular}


Table 2: Photolysis reactions (... continued)

\begin{tabular}{|c|c|c|c|c|}
\hline \# & labels & reaction & rate coefficient & reference \\
\hline AroJ109 & TrGCNJ & $\begin{array}{l}\mathrm{NDNCRESOOH}+\mathrm{h} \nu \rightarrow \mathrm{NC} 4 \mathrm{MDCO} 2 \mathrm{HN}+\mathrm{HNO}_{3}+\mathrm{CO}+\mathrm{CO} \\
+\mathrm{NO}_{2}+\mathrm{OH}\end{array}$ & jx(ip_CH3OOH) & $\frac{\text { Rickard }}{(2009)^{*}}$ \\
\hline AroJ110 & TrGCNJ & $\begin{array}{l}\mathrm{DNCRESOOH}+\mathrm{h} \nu \rightarrow \mathrm{NC} 4 \mathrm{MDCO} 2 \mathrm{HN}+\mathrm{HCOCO}_{2} \mathrm{H}+\mathrm{NO}_{2}+ \\
\mathrm{OH}\end{array}$ & jx (ip_CH3OOH) & $\frac{\text { Rickard }}{(2009)^{*}}$ \\
\hline AroJ111 & TrGCJ & $\mathrm{C} 6 \mathrm{COOHCO} 3 \mathrm{H}+\mathrm{h} \nu \rightarrow \mathrm{C} 5134 \mathrm{CO} 2 \mathrm{OH}+\mathrm{HO}_{2}+\mathrm{CO}+\mathrm{OH}$ & jx (ip_CH3OOH) & Rickard and Pascoe $(2009)$ \\
\hline AroJ112 & TrGCJ & $\mathrm{STYRENOOH}+\mathrm{h} \nu \rightarrow \mathrm{HO}_{2}+\mathrm{HCHO}+\mathrm{BENZAL}+\mathrm{OH}$ & jx (ip_CH3OOH) & $\frac{\text { Rickard }}{(2009)^{*}}$ \\
\hline
\end{tabular}

\section{*Notes:}

J-values are calculated with an external module and then supplied to the MECCA chemistry.

Values that originate from the Master Chemical Mechanism (MCM) by Rickard and Pascoe (2009) are translated according in the following way:

$\mathrm{J}(11) \rightarrow \mathrm{jx}\left(\mathrm{ip} \mathrm{C}_{-} \mathrm{COH} 2\right)$

$\mathrm{J}(12) \rightarrow \mathrm{jx}\left(\mathrm{ip} \mathrm{CHOH}_{\mathrm{C}}\right)$

$\mathrm{J}(15) \rightarrow \mathrm{jx}($ ip_HOCH2CHO $)$

$\mathrm{J}(18) \rightarrow$ jx (ip_MACR)

$\mathrm{J}(22) \rightarrow$ jx (ip_ACETOL)

$\mathrm{J}(23)+\mathrm{J}(24) \rightarrow$ jx (ip_MVK)

$\mathrm{J}(31)+\mathrm{J}(32)+\mathrm{J}(33) \rightarrow \mathrm{jx}($ ip_GLYOX $)$

$\mathrm{J}(34) \rightarrow \mathrm{jx}$ (ip_MGLYOX)

$\mathrm{J}(41) \rightarrow \mathrm{jx}$ (ip_CH3OOH)

$\mathrm{J}(53) \rightarrow \mathrm{J}\left(\mathrm{iC}_{3} \mathrm{H}_{7} \mathrm{ONO}_{2}\right)$

$\mathrm{J}(54) \rightarrow \mathrm{J}\left(\mathrm{iC}_{3} \mathrm{H}_{7} \mathrm{ONO}_{2}\right)$

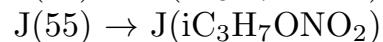

$\mathrm{J}(56)+\mathrm{J}(57) \rightarrow \mathrm{jx}$ (ip_NOA)

AroJ001: $\quad$ KDEC MCOCOMOXO $\rightarrow \mathrm{HCHO}+$ $\mathrm{CH} 3 \mathrm{CO} 3$

AroJ003: KDEC C3DIALO $\rightarrow$ GLYOX $+\mathrm{CO}+\mathrm{HO} 2$ AroJ008: KDEC MALDIALO $\rightarrow$ GLYOX + GLYOX $+\mathrm{HO} 2$

AroJ009: KDEC BZFUO $\rightarrow$ CO14O3CHO $+\mathrm{HO} 2$
AroJ011: $\quad$ KDEC NBZFUO $\rightarrow .5$ CO14O3CHO $+.5 \quad .71$ GLYOX +.29 PBZQONE + HO2

$\mathrm{NO} 2+.5 \mathrm{NBZFUONE}+.5 \mathrm{HO} 2$

AroJ013: KDEC MALDIALCO2 $\rightarrow .6$ MALANHY + $\mathrm{HO} 2+.4$ GLYOX + .4 CO

AroJ016: KDEC MALANHYO $\rightarrow$ HCOCOHCO3

AroJ027: KDEC C5DIALO $\rightarrow$ MALDIAL + CO + $\mathrm{HO} 2$

AroJ028: $\quad$ KDEC NPXYFUO $\rightarrow$ C23O3CCHO $+\mathrm{NO} 2$ AroJ030: KDEC PXYFUO $\rightarrow$ C23O3CCHO $+\mathrm{HO} 2$

AroJ032: KDEC TLFUONE $\rightarrow .6 \mathrm{C} 5 \mathrm{CO} 14 \mathrm{O} 2+.6$ $\mathrm{HO} 2+.4$ TLFUONE

AroJ036: KDEC MMALANHYO $\rightarrow$ CO2H3CO3 AroJ037: KDEC C5DICARBO $\rightarrow$ MGLYOX + GLYOX + HO2

AroJ053: KDEC NDNPHENO $\rightarrow$ NC4DCO2H + $\mathrm{HNO} 3+\mathrm{CO}+\mathrm{CO}+\mathrm{NO} 2$

AroJ054: KDEC BZBIPERO $\rightarrow$ GLYOX $+\mathrm{HO} 2+$ $.5 \mathrm{BZFUONE}+.5 \mathrm{BZFUONE}$

AroJ038: KDEC NTLFUO $\rightarrow$ ACCOMECHO $+\mathrm{NO} 2$

AroJ039: KDEC MECOACETO $\rightarrow \mathrm{CH} 3 \mathrm{CO} 3+$ $\mathrm{HCHO}$

AroJ042: KDEC C5CO14CO2 $\rightarrow .83$ MALANHY + $.83 \mathrm{CH} 3 \mathrm{O} 2+.17$ MGLYOX $+.17 \mathrm{HO} 2+.17 \mathrm{CO}$ AroJ043: KDEC C3MCODBCO2 $\rightarrow .35$ MGLYOX + $.35 \mathrm{HO} 2+.35 \mathrm{CO}+.65$ MMALANHY $+.65 \mathrm{HO} 2$ AroJ045: KDEC TLFUO $\rightarrow$ ACCOMECHO $+\mathrm{HO} 2$ AroJ048: KDEC MC3CODBCO2 $\rightarrow .35$ GLYOX + $.35 \mathrm{CH} 3 \mathrm{O} 2+.35 \mathrm{CO}+.65$ MMALANHY + .65 HO2 AroJ050: KDEC PHENO $\rightarrow .71$ MALDALCO2H +
AroJ062: $\quad$ KDEC NNCATECO $\rightarrow$ NC4DCO2H + $\mathrm{HCOCO} 2 \mathrm{H}+\mathrm{NO} 2$

AroJ065: KDEC NCATECO $\rightarrow$ NC4DCO2H + $\mathrm{HCOCO} 2 \mathrm{H}+\mathrm{HO} 2$

AroJ066: KDEC PBZQO $\rightarrow$ C5CO2OHCO3

AroJ068: KDEC BZBIPERO $\rightarrow$ GLYOX $+\mathrm{HO} 2+$ $.5 \mathrm{BZFUONE}+.5 \mathrm{BZFUONE}$

AroJ069: KDEC NBZQO $\rightarrow$ C6CO4DB $+\mathrm{NO} 2$

AroJ072: $\quad$ KDEC DNPHENO $\rightarrow$ NC4DCO2H + $\mathrm{HCOCO} 2 \mathrm{H}+\mathrm{NO} 2$

ArOJ075: KDEC BZEMUCO $\rightarrow .5$ EPXC4DIAL +.5 GLYOX $+.5 \mathrm{HO} 2+.5 \mathrm{C} 3 \mathrm{DIALO} 2+.5 \mathrm{C} 32 \mathrm{OH} 13 \mathrm{CO}$ AroJ076: $\quad$ KDEC BZEMUCO $\rightarrow .5$ EPXC4DIAL +.5 AroJ079: $\mathrm{KROPRIM}^{*} \mathrm{O} 2$ fast reaction $\mathrm{C} 6 \mathrm{H} 5 \mathrm{CH} 2 \mathrm{O}=$ BENZAL + HO2

AroJ080: KROPRIM*O2 fast reaction $\mathrm{C} 6 \mathrm{H} 5 \mathrm{CH} 2 \mathrm{O}=$ BENZAL + HO2 $\mathrm{GLYOX}+.5 \mathrm{HO} 2+.5 \mathrm{C} 3 \mathrm{DIALO} 2+.5 \mathrm{C} 32 \mathrm{OH} 13 \mathrm{CO}$ 
AroJ082: $\quad$ KDEC TLBIPERO $\rightarrow .6$ GLYOX + .4 + NO2

MGLYOX $+\mathrm{HO} 2+.2$ ZCODC23DBCOD +.2

$\mathrm{C} 5 \mathrm{DICARB}+.2 \mathrm{TLFUONE}+.2 \mathrm{BZFUONE}+.2$

MALDIAL

AroJ083: KDEC TLBIPERO $\rightarrow .6$ GLYOX +.4

MGLYOX $+\mathrm{HO} 2+.2$ ZCODC23DBCOD +.2

C5DICARB +.2 TLFUONE +.2 BZFUONE +.2

MALDIAL

AroJ085: KDEC CRESO $\rightarrow .68 \mathrm{C} 5 \mathrm{CO} 14 \mathrm{OH}+.68$

GLYOX + HO2 + .32 PTLQONE

AroJ086: $\mathrm{KDEC} \mathrm{CRESO} \rightarrow .68 \mathrm{C} 5 \mathrm{CO} 14 \mathrm{OH}+.68$ GLYOX + HO2 + .32 PTLQONE

AroJ087: KDEC NCRESO $\rightarrow$ C5CO14OH + GLYOX

\section{AroJ092:}

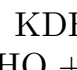
$\mathrm{HO} 2$

8

AroJ103: $\quad$ KDEC NPTLQO $\rightarrow$ C7CO4DB + NO2

AroJ104: KDEC PTLQO $\rightarrow$ C6CO2OHCO3
AroJ106: KDEC MNNCATECO $\rightarrow$ NC4MDCO2H + $\mathrm{HCOCO} 2 \mathrm{H}+\mathrm{NO} 2$

AroJ107: $\quad$ KDEC MNCATECO $\rightarrow$ NC4MDCO2H + $\mathrm{HCOCO} 2 \mathrm{H}+\mathrm{HO} 2$

$\mathrm{HNO} 3+\mathrm{CO}+\mathrm{CO}+\mathrm{NO} 2$

AroJ110: KDEC DNCRESO $\rightarrow$ NC4MDCO2H + $\mathrm{HCOCO} 2 \mathrm{H}+\mathrm{NO} 2$

AroJ112: KDEC STYRENO $\rightarrow \mathrm{HO} 2+\mathrm{HCHO}+$ BENZAL 
Table 3: Box model simulation set up

\begin{tabular}{ll} 
Variable & Value \\
\hline & $(1-10)$ th - August \\
Time period & $10^{\circ}$ south \\
Latitude & $301 \mathrm{~K}$ \\
Temperature & $101325 . \mathrm{Pa}$ \\
Pressure & $70 \%$ \\
Relative humidity & $6.666 \times 10^{10} \mathrm{mlc} \mathrm{cm}^{-2} \mathrm{~s}^{-1}$ \\
Flux of Benzene & $3.333 \times 10^{11} \mathrm{mlc} \mathrm{cm}^{-2} \mathrm{~s}^{-1}$ \\
Flux of Toluene & $6.666 \times 10^{10} \mathrm{mlc} \mathrm{cm}^{-2} \mathrm{~s}^{-1}$ \\
Flux of Phenol & $3.333 \times 10^{11} \mathrm{mlc} \mathrm{cm}^{-2} \mathrm{~s}^{-1}$ \\
Flux of NO & $6.666 \times 10^{10} \mathrm{mlc} \mathrm{cm}^{-2} \mathrm{~s}^{-1}$ \\
Flux of DMS & $6.666 \times 10^{10} \mathrm{mlc} \mathrm{cm}^{-2} \mathrm{~s}^{-1}$ \\
Flux of CH3CHO & \\
\hline
\end{tabular}


Table 4: Henry's coefficients assigned to aromatic compounds used in the chemical mechanism

\begin{tabular}{|c|c|c|c|c|}
\hline aromatic species & $\mathbf{H}[\mathrm{M} / \mathbf{a t m}]$ & $\begin{array}{l}\Delta_{\text {soln }} H / \mathbf{R} \\
{[\mathbf{K}]}\end{array}$ & specie & Reference \\
\hline BENZENE & $1.810^{-1}$ & 4100 & Benzene & $\overline{\text { Sander }}(\overline{2015})$ \\
\hline TOLUENE & $1.510^{-1}$ & 4000 & Toluene & Sander (2015) \\
\hline$B E N Z A L$ & $3.610^{1}$ & & Benzaldehyde & Sander (2015) \\
\hline MALANHY & $3.610^{1}$ & 0 & & \\
\hline TLFUONE & $3.610^{1}$ & 0 & 5-Methyl-2(5H)-furanone & \\
\hline $\mathrm{C} 6 \mathrm{H} 5 \mathrm{CH} 2 \mathrm{NO} 3$ & $3.610^{1}$ & 0 & & \\
\hline BZFUONE & $3.610^{1}$ & 0 & & \\
\hline PXYFUONE & $3.610^{1}$ & 0 & 3-Methyl-2(5H)-furanone & \\
\hline PHENOL & $2.910^{3}$ & 6800 & Phenol & Sander (2015) \\
\hline $\mathrm{C} 6 \mathrm{H} 5 \mathrm{O}$ & $2.910^{3}$ & 6800 & Phenyloxidanyl & \\
\hline $\mathrm{C} 6 \mathrm{H} 5 \mathrm{OOH}$ & $2.910^{3}$ & 6800 & Phenyl hydroperoxide & \\
\hline $\mathrm{C} 6 \mathrm{H} 5 \mathrm{CH} 2 \mathrm{OOH}$ & $2.910^{3}$ & 6800 & Benzyl hydroperoxide & \\
\hline TOL1O & $2.910^{3}$ & 6800 & (2-Methylphenyl)oxidanyl & \\
\hline $\mathrm{C} 6 \mathrm{H} 5 \mathrm{CH} 2 \mathrm{OH}$ & $2.910^{3}$ & 6800 & & \\
\hline OXYL1OOH & $2.910^{3}$ & 6800 & & \\
\hline BZBIPERNO3 & $2.910^{3}$ & 6800 & & \\
\hline CRESOL & $2.910^{3}$ & 6800 & Cresol & \\
\hline $\mathrm{HOC}_{4} \mathrm{H}_{4} \mathrm{NO} 2$ & $7.010^{1}$ & 4600 & 2-nitrophenol & Sander (2015) \\
\hline NPHEN1OOH & $7.010^{1}$ & 4600 & & \\
\hline NPHEN1O & $7.010^{1}$ & 4600 & & \\
\hline TOL1OHNO2 & $7.010^{1}$ & 4600 & & \\
\hline NCRES1O & $7.010^{1}$ & 4600 & & \\
\hline NCRES1OOH & $7.010^{1}$ & 4600 & & \\
\hline TLBIPERNO3 & $7.010^{1}$ & 4600 & & \\
\hline PBZN & $7.010^{1}$ & 4600 & benzoyl nitro peroxide & \\
\hline NSTYRENOOH & $7.010^{1}$ & 4600 & & \\
\hline DNPHEN & $2.310^{3}$ & 0 & dinitro-o-cresol & Sander (2015) \\
\hline NDNPHENOOH & $2.310^{3}$ & 0 & & \\
\hline NNCATECOOH & $2.310^{3}$ & 0 & & \\
\hline
\end{tabular}


Table 4: Henry's coefficients (... continued)

\begin{tabular}{|c|c|c|c|c|}
\hline aromatic species & $\mathbf{H}[\mathrm{M} /$ atm $]$ & $\begin{array}{l}\Delta_{\text {soln }} H / \mathbf{R} \\
{[\mathbf{K}]}\end{array}$ & specie & Reference \\
\hline DNPHENOOH & $2.310^{3}$ & 0 & & \\
\hline MNNCATCOOH & $2.310^{3}$ & 0 & & \\
\hline DNCRESOOH & $2.310^{3}$ & 0 & & \\
\hline NDNCRESOOH & $2.310^{3}$ & 0 & & \\
\hline DNCRES & $2.310^{3}$ & 0 & 2-Methyl-4,6-dinitrophenol & \\
\hline $\mathrm{PHCOOH}$ & $2.410^{4}$ & 0 & Benzoic Acid & Sander (2015) \\
\hline PTLQCO & $2.410^{4}$ & 0 & & \\
\hline $\mathrm{C} 6 \mathrm{H} 5 \mathrm{CO} 3 \mathrm{H}$ & $2.410^{4}$ & 0 & Perbenzoic acid & \\
\hline NBZQOOH & $2.410^{4}$ & 0 & & \\
\hline NPTLQOOH & $2.410^{4}$ & 0 & & \\
\hline NBZFUOOH & $2.410^{4}$ & 0 & & \\
\hline STYRENOOH & $2.410^{4}$ & 0 & & \\
\hline CATECHOL & $4.610^{3}$ & 0 & Catechol & Sander (2015) \\
\hline PBZQCO & $4.610^{3}$ & 0 & & \\
\hline NPHENOH & $4.610^{3}$ & 0 & & \\
\hline CATEC1O & $4.610^{3}$ & 0 & & \\
\hline NPHENOOH & $4.610^{3}$ & 0 & & \\
\hline NCRESOOH & $4.610^{3}$ & 0 & & \\
\hline NCATECHOL & $4.610^{3}$ & 0 & & \\
\hline CATEC1OOH & $4.610^{3}$ & 0 & & \\
\hline MNCATECH & $4.610^{3}$ & 0 & 3-Methyl-6-nitro-1,2-benzenediol & \\
\hline MCATECHOL & $4.610^{3}$ & 0 & 3-Methylcatechol & \\
\hline MCATEC1OOH & $4.610^{3}$ & 0 & & \\
\hline NCRESOH & $4.610^{3}$ & 0 & & \\
\hline GLYOX & $3.610^{5}$ & 0 & Glyoxal & Sander (2015) \\
\hline C5DIALOOH & $3.610^{5}$ & 0 & & \\
\hline BZEPOXMUC & $3.610^{5}$ & 0 & & \\
\hline MALDIAL & $3.610^{5}$ & 0 & 2-Butenedial & \\
\hline $\mathrm{CO} 14 \mathrm{O} 3 \mathrm{CHO}$ & $3.610^{5}$ & 0 & & \\
\hline $\mathrm{CO} 23 \mathrm{C} 3 \mathrm{CHO}$ & $3.610^{5}$ & 0 & & \\
\hline $\mathrm{C} 54 \mathrm{CO}$ & $3.610^{5}$ & 0 & 2,3,4-Trioxopentanal & \\
\hline
\end{tabular}


Table 4: Henry's coefficients (... continued)

\begin{tabular}{|c|c|c|c|c|}
\hline aromatic species & $\mathbf{H}[\mathrm{M} / \mathrm{atm}]$ & $\begin{array}{l}\Delta_{\text {soln }} H / \mathbf{R} \\
{[\mathbf{K}]}\end{array}$ & specie & Reference \\
\hline EPXC4DIAL & $3.610^{5}$ & 0 & & \\
\hline$M G L Y O X$ & $3.710^{3}$ & 7500 & Methyl glyoxal & Sander (2015) \\
\hline C5CO2DBPAN & $3.710^{3}$ & 7500 & & \\
\hline C7CO4DB & $3.710^{3}$ & 7500 & & \\
\hline GLYPAN & $3.710^{3}$ & 7500 & & \\
\hline METACETHO & $3.710^{3}$ & 7500 & & \\
\hline C23O3CHO & $3.710^{3}$ & 7500 & & \\
\hline $\mathrm{C} 23 \mathrm{O} 3 \mathrm{CCHO}$ & $3.710^{3}$ & 7500 & & \\
\hline C5DICARB & $3.710^{3}$ & 7500 & & \\
\hline АCCOMECHO & $3.710^{3}$ & 7500 & & \\
\hline C4MDIAL & $3.710^{3}$ & 7500 & & \\
\hline $\mathrm{C} 6125 \mathrm{CO}$ & $3.710^{3}$ & 7500 & 2,5-Dioxo-3-hexenal & \\
\hline$P A N$ & $2.810^{0}$ & 6500 & peroxyacetylnitrate & Sander (2015) \\
\hline $\mathrm{C} 5 \mathrm{COO} 2 \mathrm{NO} 2$ & $2.810^{0}$ & 6500 & & \\
\hline MC3CODBPAN & $2.810^{0}$ & 6500 & & \\
\hline EPXDLPAN & $2.810^{0}$ & 6500 & & \\
\hline ACCOMEPAN & $2.810^{\circ}$ & 6500 & & \\
\hline C3MCODBPAN & $2.810^{0}$ & 6500 & & \\
\hline C23O3CPAN & $2.810^{0}$ & 6500 & & \\
\hline BZEMUCPAN & $2.810^{0}$ & 6500 & & \\
\hline TLEMUCPAN & $2.810^{0}$ & 6500 & & \\
\hline MALDIALPAN & $2.810^{0}$ & 6500 & & \\
\hline CH3O2NO2 & $2.810^{0}$ & 6500 & (Nitroperoxy)methane & \\
\hline PHAN & $3.910^{4}$ & 8600 & & Sander (2015) \\
\hline TLOBIPEROH & $3.910^{4}$ & 8600 & & \\
\hline C4CO2DBPAN & $3.910^{4}$ & 8600 & & \\
\hline HCOCOHPAN & $3.910^{4}$ & 8600 & & \\
\hline $\mathrm{NC} 4 \mathrm{DCO} 2 \mathrm{H}$ & $3.910^{4}$ & 8600 & & \\
\hline C4PAN6 & $3.910^{4}$ & 8600 & & \\
\hline C5CO2OHPAN & $3.910^{4}$ & 8600 & & \\
\hline BZEMUCNO3 & $3.910^{4}$ & 8600 & & \\
\hline
\end{tabular}


Table 4: Henry's coefficients (... continued)

\begin{tabular}{|c|c|c|c|c|}
\hline aromatic species & $\mathbf{H}[\mathrm{M} / \mathrm{atm}]$ & $\begin{array}{l}\Delta_{\text {soln }} H / \mathbf{R} \\
{[\mathbf{K}]}\end{array}$ & specie & Reference \\
\hline TLEMUCNO3 & $3.910^{4}$ & 8600 & & \\
\hline C6CO2OHPAN & $3.910^{4}$ & 8600 & & \\
\hline СНЗООН & $3.210^{2}$ & 5300 & methyl hydroperoxide & Sander 2015) \\
\hline 3-Hexanol & $2.010^{1}$ & & & Sander (2015) \\
\hline 2,5-hexanediol & $2.010^{6}$ & & & Sander $(2015)$ \\
\hline C4M2AL2OH & $2.010^{6}$ & & & \\
\hline C4MALOHOOH & $2.010^{6}$ & & & \\
\hline С6COOHCO3H & $2.010^{6}$ & & & \\
\hline TLEMUCOOH & $2.010^{6}$ & & & \\
\hline $\mathrm{HCOCOHCO} 3 \mathrm{H}$ & $2.010^{6}$ & & & \\
\hline MALDIALOOH & $2.010^{6}$ & & & \\
\hline MMALNHY2OH & $2.010^{6}$ & & & \\
\hline PHENOH & $2.010^{6}$ & & & \\
\hline MNCATECOOH & $2.010^{6}$ & & & \\
\hline BZFUOOH & $2.010^{6}$ & & & \\
\hline MALNHYOHCO & $2.010^{6}$ & & & \\
\hline MALANHYOOH & $2.010^{6}$ & & & \\
\hline MALANHY2OH & $2.010^{6}$ & & & \\
\hline BZFUOH & $2.010^{6}$ & & & \\
\hline PXYFUOOH & $2.010^{6}$ & & & \\
\hline TLFUOH & $2.010^{6}$ & & & \\
\hline MMALNHYOOH & $2.010^{6}$ & & & \\
\hline PXYFUOH & $2.010^{6}$ & & & \\
\hline TLFUOOH & $2.010^{6}$ & & & \\
\hline PHENOOH & $2.010^{6}$ & & & \\
\hline NCATECOOH & $2.010^{6}$ & & & \\
\hline PBZQOOH & $2.010^{6}$ & & & \\
\hline BZBIPEROOH & $2.010^{6}$ & & & \\
\hline BZBIPER2OH & $2.010^{6}$ & & & \\
\hline PBZQOH & $2.010^{6}$ & & & \\
\hline PTLQOH & $2.010^{6}$ & & & \\
\hline
\end{tabular}


Table 4: Henry's coefficients (... continued)

\begin{tabular}{|c|c|c|c|c|}
\hline aromatic species & $\mathbf{H}[\mathrm{M} / \mathrm{atm}]$ & $\begin{array}{l}\Delta_{\text {soln }} H / \mathbf{R} \\
{[\mathbf{K}]}\end{array}$ & specie & Reference \\
\hline CRESOH & $2.010^{6}$ & & & \\
\hline TLBIPER2OH & $2.010^{6}$ & & & \\
\hline TLEMUCOH & $2.010^{6}$ & & & \\
\hline TLBIPEROOH & $2.010^{6}$ & & & \\
\hline MCATEC1O & $2.010^{6}$ & & & \\
\hline PTLQOOH & $2.010^{6}$ & & & \\
\hline CRESOOH & $2.010^{6}$ & & & \\
\hline HOHOC4DIAL & $2.010^{6}$ & & & \\
\hline CO2C4DIAL & $2.010^{6}$ & & & \\
\hline $\mathrm{C} 514 \mathrm{CO} 23 \mathrm{OH}$ & $2.010^{6}$ & & & \\
\hline C5DICAROOH & $2.010^{6}$ & & & \\
\hline $\mathrm{C} 6 \mathrm{CO} 4 \mathrm{DB}$ & $2.010^{6}$ & & & \\
\hline BZEMUCOOH & $2.010^{6}$ & & & \\
\hline BZEMUCOH & $2.010^{6}$ & & & \\
\hline $\mathrm{C} 5 \mathrm{COOHCO} 3 \mathrm{H}$ & $2.010^{6}$ & & & \\
\hline $\mathrm{C} 4 \mathrm{CO} 2 \mathrm{DCO} 3 \mathrm{H}$ & $2.010^{6}$ & & & \\
\hline $\mathrm{C} 5 \mathrm{CO} 2 \mathrm{DCO} 3 \mathrm{H}$ & $2.010^{6}$ & & & \\
\hline 1,2,6-hexanetriol & $2.010^{11}$ & & & Sander (2015) \\
\hline trans-2-hexenal & $2.010^{1}$ & & & Sander (2015) \\
\hline MMALANHY & $2.010^{1}$ & & & \\
\hline PBZQONE & $2.010^{1}$ & & 1,4-Benzoquinone & \\
\hline NBZFUONE & $2.010^{1}$ & & & \\
\hline Glyoxylic acid & $9.010^{3}$ & & & Sander (2015) \\
\hline $\mathrm{NC} 4 \mathrm{MDCO} 2 \mathrm{H}$ & $9.010^{3}$ & & & \\
\hline BZEMUCCO2H & $9.010^{3}$ & & & \\
\hline BZEMUCCO & $9.010^{3}$ & & & \\
\hline BZEMUCCO3H & $9.010^{3}$ & & & \\
\hline BZFUCO & $9.010^{3}$ & & & \\
\hline MCOCOMOOOH & $9.010^{3}$ & & & \\
\hline $\mathrm{CO} 14 \mathrm{O} 3 \mathrm{CO} 2 \mathrm{H}$ & $9.010^{3}$ & & & \\
\hline BZOBIPEROH & $9.010^{3}$ & & & \\
\hline
\end{tabular}


Table 4: Henry's coefficients (... continued)

\begin{tabular}{|c|c|c|c|c|}
\hline aromatic species & $\mathbf{H}[\mathbf{M} / \mathbf{a t m}]$ & $\begin{array}{l}\Delta_{\text {soln }} H / \mathbf{R} \\
{[\mathbf{K}]}\end{array}$ & specie & Reference \\
\hline NTLFUOOH & $9.010^{3}$ & & & \\
\hline $\mathrm{C} 33 \mathrm{CO}$ & $9.010^{3}$ & & Oxomalonaldehyde & \\
\hline C3DIALOOH & $9.010^{3}$ & & & \\
\hline C5DIALCO & $9.010^{3}$ & & & \\
\hline С $32 \mathrm{OH} 13 \mathrm{CO}$ & $9.010^{3}$ & & & \\
\hline MALDALCO3H & $9.010^{3}$ & & & \\
\hline EPXDLCO3H & $9.010^{3}$ & & & \\
\hline $\mathrm{C} 615 \mathrm{CO} 2 \mathrm{OH}$ & $9.010^{3}$ & & & \\
\hline EPXDLCO2H & $9.010^{3}$ & & & \\
\hline MALDALCO2H & $9.010^{3}$ & & 4-Oxo-2-butenoic acid & \\
\hline C5DIALOH & $9.010^{3}$ & & & Sander (2015) \\
\hline MC3ODBCO3H & $9.010^{3}$ & & & \\
\hline СНзСОСО2Н & $3.110^{5}$ & 5100 & Piruvic Acid & Sander (2015) \\
\hline $\mathrm{C} 5134 \mathrm{CO} 2 \mathrm{OH}$ & $3.110^{5}$ & 5100 & & \\
\hline $\mathrm{C} 23 \mathrm{O} 3 \mathrm{CCO} 2 \mathrm{H}$ & $3.110^{5}$ & 5100 & & \\
\hline $\mathrm{C} 24 \mathrm{O} 3 \mathrm{CCO} 2 \mathrm{H}$ & $3.110^{5}$ & 5100 & & \\
\hline MECOACEOOH & $3.110^{5}$ & 5100 & & \\
\hline C5CO14OOH & $3.110^{5}$ & 5100 & & \\
\hline $\mathrm{C} 4 \mathrm{CODBCO} 3 \mathrm{H}$ & $3.110^{5}$ & 5100 & & \\
\hline $\mathrm{C} 23 \mathrm{O} 3 \mathrm{CCO} 3 \mathrm{H}$ & $3.110^{5}$ & 5100 & & \\
\hline ACCOMECO3H & $3.110^{5}$ & 5100 & & \\
\hline $\mathrm{C} 615 \mathrm{CO} 2 \mathrm{OOH}$ & $3.110^{5}$ & 5100 & & \\
\hline СH3COCO3H & $3.110^{5}$ & 5100 & & \\
\hline HOCOC4DIAL & $3.110^{5}$ & 5100 & & \\
\hline TLEMUCCO & $3.110^{5}$ & 5100 & & \\
\hline pentanoic acid & $2.210^{3}$ & 6583 & & Sander $(2015)$ \\
\hline $\mathrm{C} 5 \mathrm{CO} 14 \mathrm{OH}$ & $2.210^{3}$ & 6583 & & \\
\hline MC3ODBCO2H & $2.210^{3}$ & 6583 & & \\
\hline TLEMUCCO2H & $2.210^{3}$ & 6583 & & \\
\hline TLEMUCCO3H & $2.210^{3}$ & 6583 & & \\
\hline NPXYFUOOH & $2.210^{3}$ & 6583 & & \\
\hline
\end{tabular}


Table 4: Henry's coefficients (... continued)

\begin{tabular}{|c|c|c|c|c|}
\hline aromatic species & $\mathbf{H}[\mathrm{M} / \mathbf{a t m}]$ & $\begin{array}{l}\Delta_{\text {soln }} H / \mathbf{R} \\
{[\mathbf{K}]}\end{array}$ & specie & Reference \\
\hline$M V K$ & $4.110^{1}$ & & & Sander (2015) \\
\hline TLEPOXMUC & $4.110^{1}$ & & & \\
\hline PTLQONE & $4.110^{1}$ & & 2-Methyl-1,4-benzoquinone & \\
\hline TLEMUCCO & $4.110^{4}$ & & & Warneck (2005) \\
\hline LXYL & $1.710^{-1}$ & & xylene (averaged) & \\
\hline $\mathrm{O}$ & $1.910^{-1}$ & & o-xylene & Sander (2015) \\
\hline M & $1.610^{-1}$ & & m-xylene & Sander $(\overline{2015})$ \\
\hline $\mathrm{P}$ & $1.610^{-1}$ & & p-xylene & $\overline{\text { Sander }}(\overline{2015})$ \\
\hline LTMB & $2.210^{-1}$ & & trimethylbenzene (ave) & \\
\hline $1,2,3$ & $3.110^{-1}$ & & 1,2,3-trimethylbenzene & Sander 2015) \\
\hline $1,2,4$ & $1.710^{-1}$ & & 1,2,4-trimethylbenzene & Sander $(\overline{2015})$ \\
\hline $1,3,5$ & $1.710^{-1}$ & & 1,2,5-trimethylbenzene & Sander (2015) \\
\hline EBENZ & $1.210^{-1}$ & & ethylbenzene & Sander 2015) \\
\hline HAROM & $1.210^{-1}$ & & 3,5-dimethyl ethyl benzene & Sander (2015) \\
\hline STYRENE & $3.710^{-1}$ & & & Sander (2015) \\
\hline
\end{tabular}

The henry's coefficient is expressed as H (M/atm). The value that are assigned are taken from the compilation of Henry's coefficients by Rolf Sander unless specified otherwise. Many species are managed choosing surrogate species. Those species that have $\mathrm{H}>10^{8}$ need to be treated in SCAV as if they had $\mathrm{H}=10^{8}$ in order to avoid too high numerical stiffness.

The first row of each box is written in italics. This row is the reference structure value and chemical structure in which we have based our approximation for other species. The criteria for choosing the Henry's coefficient for those species which is not defined is summarized in the following points:

1. Non-aromatic ring products: Hexanol (1OH), Hexanediol (2OH or $\mathrm{OOH})$, Hexantril(3OH)

2. Conjugated carbonyls: Glyox (Doble aldehyde), Crotonaldehyde/trans-2-hexena (C5dicarb), 3-methyl butanoic acid $(=\mathrm{O}+-\mathrm{OH})$

3. glyoxilic acid (aldehyde+carbonyl) 
4. For species with more of 2-OH we use 10E6, which in the model is enough for an uptake of 90.

5. - $\mathrm{O}$ - we consider it inert (epoxy group doesn't contribute efficiently. Epoxypropane $\mathrm{Hr}=5.3$ )

6. Not Hr for peroxy radicals (they react enough fast). They are not shown in the table.

7. We will tend to attach species to those of higher Rh (in case of doubt) 


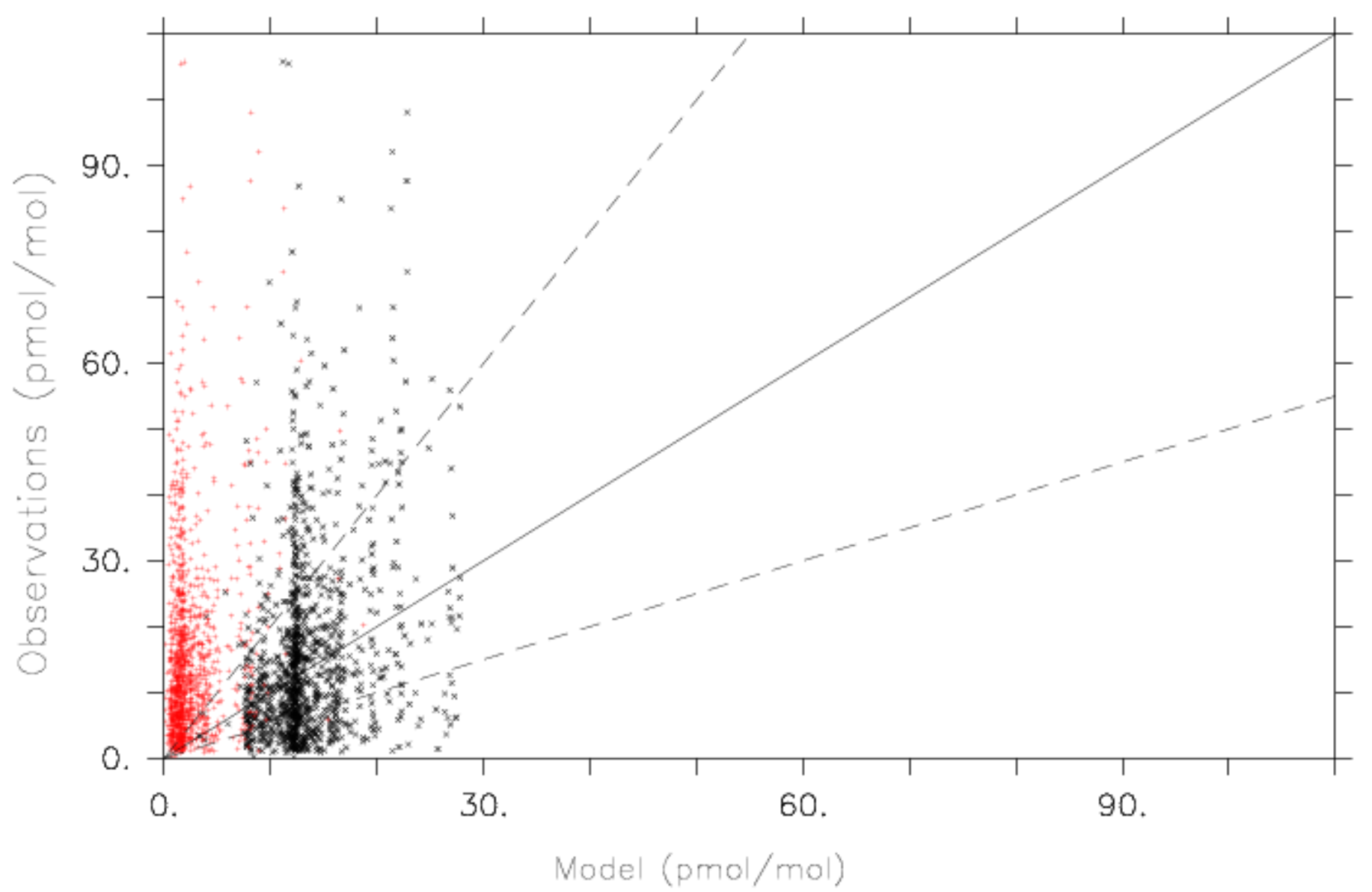

Figure 1: Scatter diagram of CARIBIC vs simulations (for benzene). In red LIT scenario, in black RCP scenario. Units are in pmol/mol 

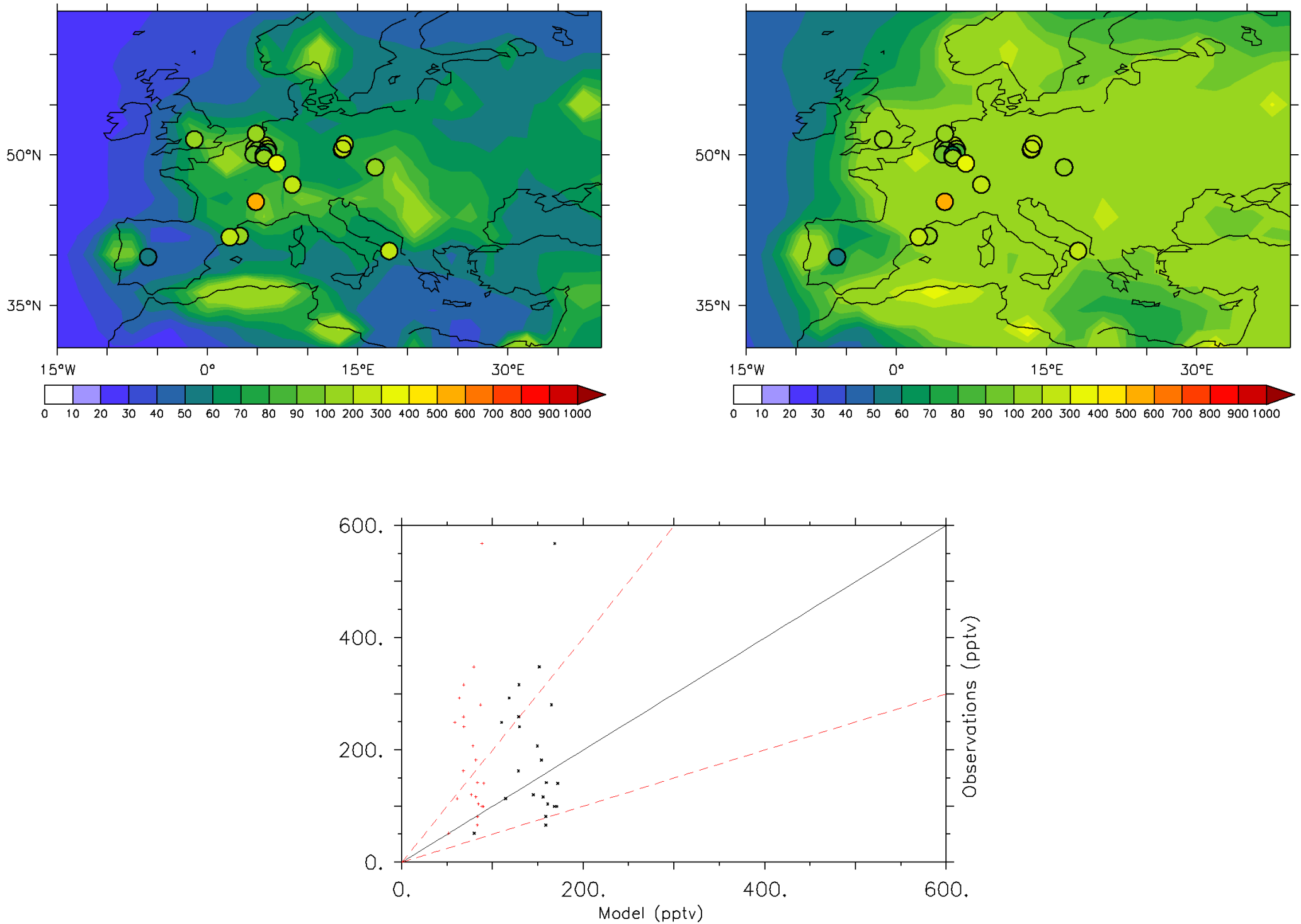

Figure 2: On top, benzene annual averaged mixing ratios for Europe (left LIT scenario, right RCP scenario). In circles, annual average mixing ratios of EEA dataset. Units are in pmol $/ \mathrm{mol}$. On the bottom, scatter plot of observations vs simulations. In red LIT scenario, in black RCP. 


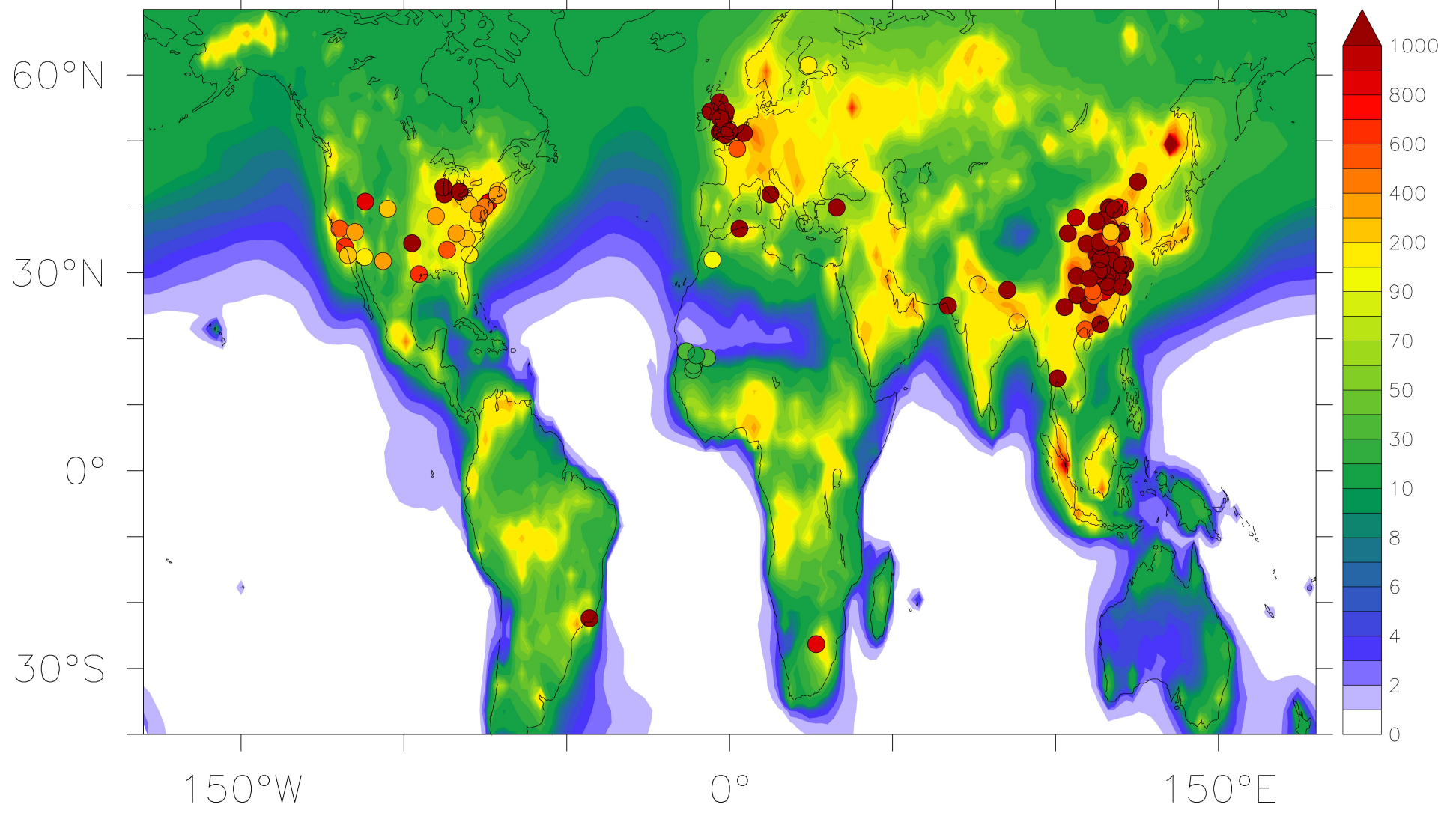

Figure 3: Toluene mixing ratios from literature in $\mathrm{pmol} / \mathrm{mol}$ (each work covers different time spans). In background model annual average mixing ratios. 

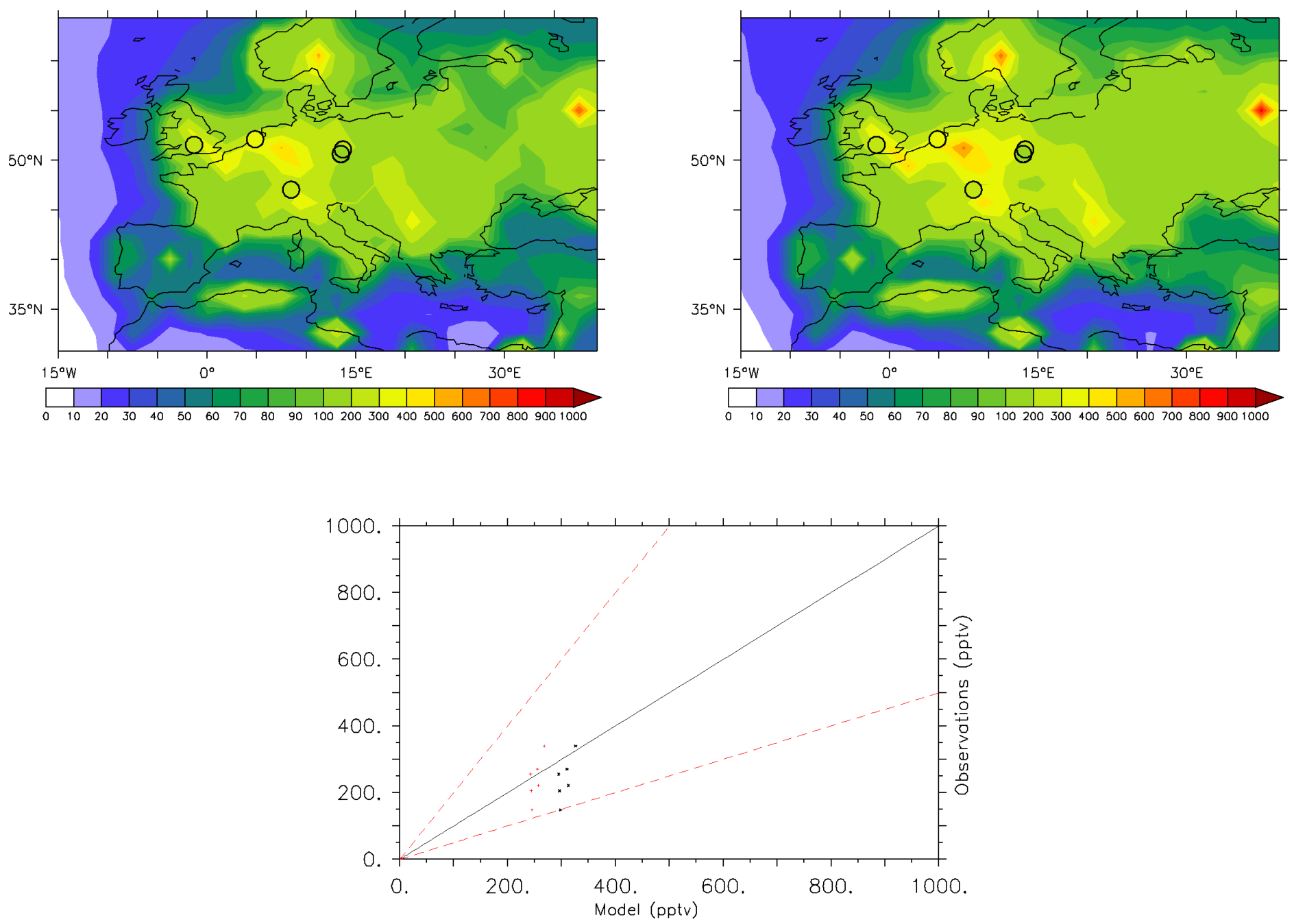

Figure 4: On top, toluene annual averaged mixing ratios for Europe (left LIT scenario, right RCP scenario). In circles, annual average mixing ratios of EEA. Units are in $\mathrm{pmol} / \mathrm{mol}$. On the bottom, scatter plot, in red LIT scenario, in black RCP 

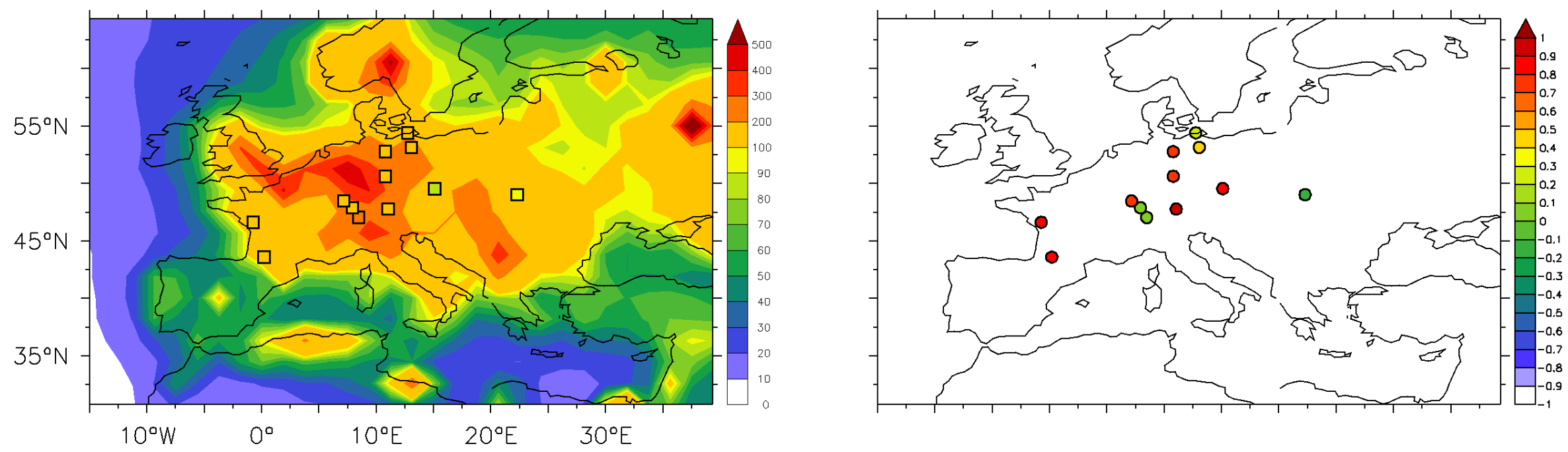

Figure 5: Left: In squares, toluene annual averaged mixing ratios of EMEP stations, in background LIT scenario annual averaged mixing ratios (in units of pmol/mol). Right: Temporal correlation between observations and simulated mixing ratios. 

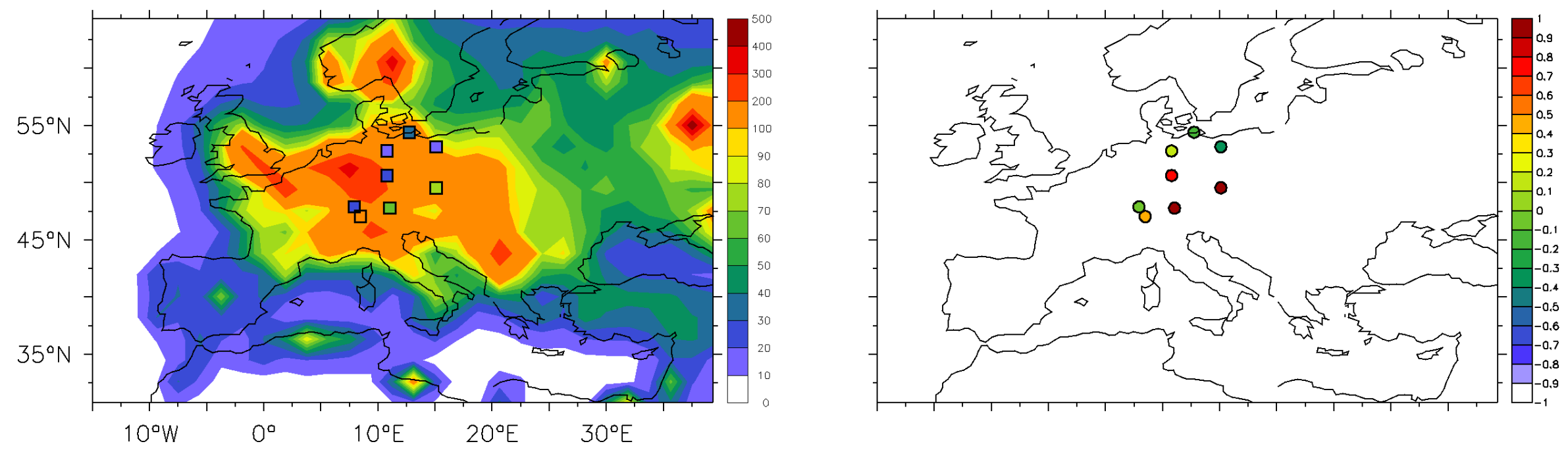

Figure 6: Left: In squares, xylene annual averaged mixing ratios of EMEP stations, in background LIT scenario annual averaged mixing ratios (in units of pmol/mol). Right: Temporal correlation between observations and simulated mixing ratios. 


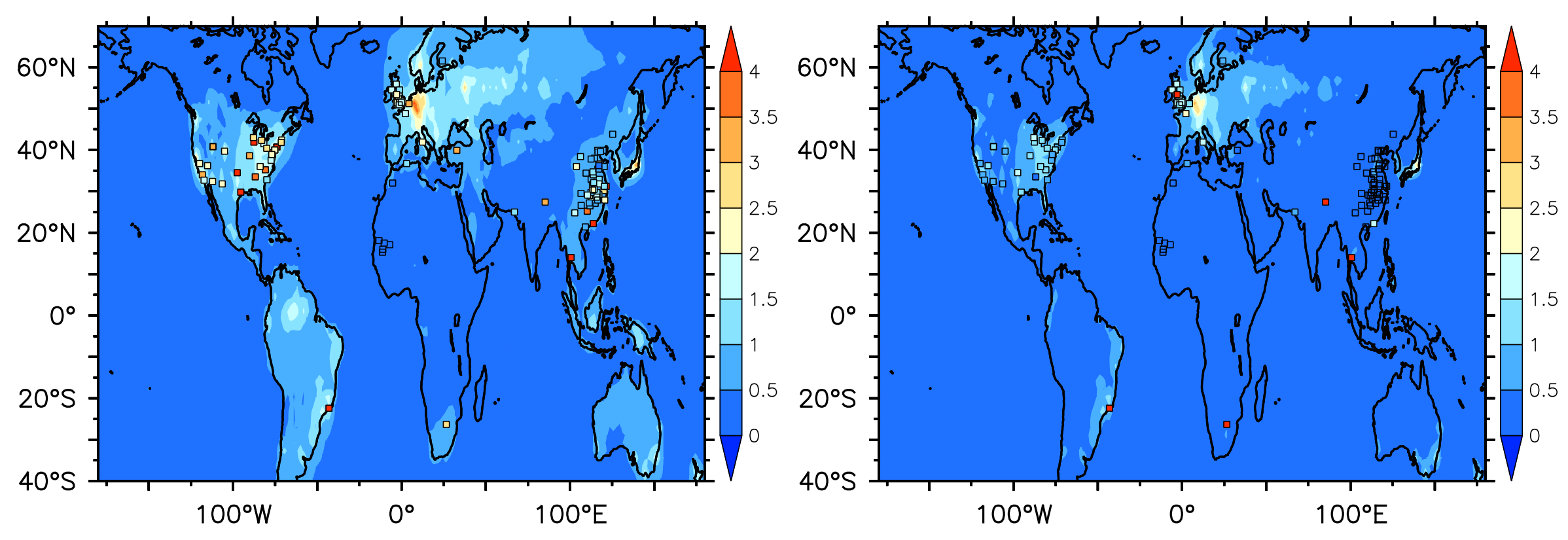

Figure 7: Left: In squares, toluene to benzene ratios, in background RCP scenario ratios. Right: Same but for xylene to benzene ratios. 


\section{References}

Atkinson, R., Baulch, D. L., Cox, R. A., Crowley, J. N., Hampson, Jr., R. F., Hynes, R. G., Jenkin, M. E., Kerr, J. A., Rossi, M. J., and Troe, J.: Summary of evaluated kinetic and photochemical data for atmospheric chemistry: Web version March 2005, http://iupac.pole-ether.fr, 2005.

Atkinson, R., Baulch, D. L., Cox, R. A., Hampson, Jr., R. F., Kerr, J. A., Rossi, M. J., and Troe, J.: Summary of evaluated kinetic and photochemical data for atmospheric chemistry: Web version August 2014, http://iupac.pole-ether.fr, 2012.

Groß, C. B. M.: Kinetische Studien zur OH-Bildung über die Reaktionen von HO 2 mit organischen Peroxyradikalen, Ph.D. thesis, Universitätsbibliothek Mainz, 2013.

Jagiella, S. and Zabel, F.: Reaction of phenylperoxy radicals with NO 2 at 298 K, Physical Chemistry Chemical Physics, 9, 5036-5051, 2007.

Liu, Y. J., Herdlinger-Blatt, I., McKinney, K. A., and Martin, S. T.: Production of methyl vinyl ketone and methacrolein via the hydroperoxyl pathway of isoprene oxidation, Atmospheric Chemistry and Physics, 13, 5715-5730, doi:10.5194/acp-13-5715-2013, http://www.atmos-chem-phys.net/13/5715/2013/, 2013.

Rickard, A. and Pascoe, S.: The Master Chemical Mechanism (MCM), http://mcm.leeds.ac.uk, 2009.

Roth, E., Chakir, A., and Ferhati, A.: Study of a Benzoylperoxy Radical in the Gas Phase: Ultraviolet Spectrum and C6H5C (O) O2+ HO2 Reaction between 295 and 357 K, The Journal of Physical Chemistry A, 114, 10367-10 379, 2010.

Sander, R.: Compilation of Henry's law constants (version 4.0) for water as solvent, Atmospheric Chemistry and Physics, 15, 4399-4981, doi:10.5194/acp-15-4399-2015, http://www.atmos-chem-phys.net/15/4399/2015/, 2015.

Taraborrelli, D., Lawrence, M. G., Butler, T. M., Sander, R., and Lelieveld, J.: Mainz Isoprene Mechanism 2 (MIM2): an isoprene oxidation mechanism for regional and global atmospheric modelling, Atmos. Chem. Phys., 9, 2751-2777, http://www.atmos-chem-phys.net/9/2751, 2009.

Warneck, P.: Multi-phase chemistry of C2 and C3 organic compounds in the marine atmosphere, Journal of Atmospheric Chemistry, 51, 119-159, 2005. 